\title{
Early-Type Galaxies in the Sloan Digital Sky Survey. I. The Sample
}

\section{Citation}

Bernardi, Mariangela, Ravi K. Sheth, James Annis, Scott Burles, Daniel J. Eisenstein, Douglas P. Finkbeiner, David W. Hogg, et al. 2003. "Early-Type Galaxies in the Sloan Digital Sky Survey. I. The Sample." The Astronomical Journal 125 (4) (April): 1817-1848. doi:10.1086/367776.

\section{Published Version}

$10.1086 / 367776$

\section{Permanent link}

http://nrs.harvard.edu/urn-3:HUL.InstRepos:33461904

\section{Terms of Use}

This article was downloaded from Harvard University's DASH repository, and is made available under the terms and conditions applicable to Other Posted Material, as set forth at http:// nrs.harvard.edu/urn-3:HUL.InstRepos:dash.current.terms-of-use\#LAA

\section{Share Your Story}

The Harvard community has made this article openly available.

Please share how this access benefits you. Submit a story.

Accessibility 


\author{
EARLY-TYPE GALAXIES IN THE SLOAN DIGITAL SKY SURVEY. I. THE SAMPLE \\ Mariangela Bernardi, ${ }^{1,2}$ Ravi K. Sheth,,${ }^{3,4}$ James Annis, ${ }^{3}$ Scott Burles, ${ }^{3}$ Daniel J. Eisenstein, ${ }^{5}$ \\ Douglas P. Finkbeiner, ${ }^{6,7,8}$ David W. Hogg, ${ }^{9}$ Robert H. Lupton, ${ }^{7}$ David J. Schlegel, ${ }^{7}$ \\ Mark SubbaRao, ${ }^{1}$ Neta A. Bahcall,${ }^{7}$ John P. Blakeslee, ${ }^{10}$ J. Brinkmann,${ }^{11}$ \\ Francisco J. Castander, ${ }^{12,13}$ Andrew J. Connolly,${ }^{4}$ István Csabai, ${ }^{10,14}$ Mamoru Doi,,${ }^{15,16}$ \\ Masataka Fukugita, ${ }^{17,18}$ Joshua Frieman, ${ }^{1,3}$ Timothy Heckman, ${ }^{10}$ Gregory S. Hennessy, ${ }^{19}$ \\ Željko Ivezić, ${ }^{7}$ G. R. Knapp,${ }^{7}$ Don Q. Lamb, ${ }^{1}$ Timothy McKay, ${ }^{20}$ Jeffrem A. Munn, ${ }^{19}$ \\ Robert Nichol, ${ }^{2}$ Sadanori OKamura, ${ }^{16,21}$ Donald P. Schneider, ${ }^{22}$ \\ ANIRUdDHa R. ThaKar, ${ }^{10}$ AND Donald G. YORK ${ }^{1}$ \\ Received 2001 October 15; accepted 2002 December 3
}

\begin{abstract}
A sample of nearly 9000 early-type galaxies, in the redshift range $0.01 \leq z \leq 0.3$, was selected from the Sloan Digital Sky Survey (SDSS) using morphological and spectral criteria. This paper describes how the sample was selected, presents examples of images and seeing-corrected fits to the observed surface brightness profiles, describes our method for estimating $K$-corrections, and shows that the SDSS spectra are of sufficiently high quality to measure velocity dispersions accurately. It also provides catalogs of the measured photometric and spectroscopic parameters. In related papers, these data are used to study how early-type galaxy observables, including luminosity, effective radius, surface brightness, color, and velocity dispersion, are correlated with one another.
\end{abstract}

Key words: galaxies: elliptical and lenticular, $\mathrm{cD}$ — galaxies: evolution -

galaxies: fundamental parameters — galaxies: photometry — galaxies: stellar content

On-line material: color figures, machine-readable tables

${ }^{1}$ Department of Astronomy and Astrophysics, University of Chicago, 5640 South Ellis Avenue, Chicago, IL 60637.

${ }^{2}$ Department of Physics, Carnegie Mellon University, 5000 Forbes Avenue, Pittsburgh, PA 15213.

${ }^{3}$ Fermi National Accelerator Laboratory, P.O. Box 500, Batavia, IL 60510.

${ }^{4}$ Department of Physics and Astronomy, University of Pittsburgh, 3941 O’Hara Street, Pittsburgh, PA 15620.

${ }^{5}$ Steward Observatory, University of Arizona, 933 North Cherry Avenue, Tucson, AZ 85121.

${ }^{6}$ Department of Astronomy, 601 Campbell Hall, University of California, Berkeley, Berkeley, CA 94720.

${ }^{7}$ Princeton University Observatory, Peyton Hall, Princeton, NJ 08544.

${ }^{8}$ Hubble Fellow.

${ }^{9}$ Department of Physics, New York University, 4 Washington Place, New York, NY 10003

${ }^{10}$ Department of Physics and Astronomy, Johns Hopkins University, 3400 North Charles Street, Baltimore, MD 21218-2686.

${ }^{11}$ Apache Point Observatory, P.O. Box 59, Sunspot, NM 88349.

12 Department of Physics, Yale University, P.O. Box 208101, New Haven, CT 06520.

${ }^{13}$ Departamento de Astronomía, Universidad de Chile, Casilla 36-D, Santiago, Chile.

${ }^{14}$ Department of Physics of Complex Systems, Eötvös Loránd University, Pf. 32, H-1117 Budapest, Hungary.

${ }^{15}$ Institute of Astronomy, School of Science, University of Tokyo, Mitaka, Tokyo 181-0015, Japan.

${ }^{16}$ Research Center for the Early Universe, School of Science, University of Tokyo, Tokyo 113-0033, Japan

${ }^{17}$ Institute for Cosmic Ray Research, University of Tokyo, Kashiwa 277-8582, Japan.

${ }^{18}$ Institute for Advanced Study, Einstein Drive, Princeton, NJ 08540.

19 US Naval Observatory, 3450 Massachusetts Avenue, NW, Washington, DC 20392-5420.

${ }^{20}$ Department of Physics, University of Michigan, 500 East University, Ann Arbor, MI 48109.

${ }^{21}$ Department of Astronomy and Astrophysics, 525 Davey Laboratory, Pennsylvania State University, University Park, PA 16802.

22 Department of Astronomy, University of Tokyo, Tokyo 113-0033, Japan.

\section{INTRODUCTION}

Galaxies have a wide range of luminosities, colors, masses, sizes, surface brightnesses, morphologies, star formation histories, and environments. This heterogeneity is not surprising, given the variety of physical processes that likely influence their formation and evolution, including gravitational collapse, hydrodynamics, turbulence, magnetic fields, black hole formation and accretion, nuclear activity, tidal and merger interactions, and evolving and inhomogeneous cosmic radiation fields.

What is surprising is that populations of galaxies show several very precise relationships among their measured properties. The properties we use to describe galaxies span a large "configuration space," but galaxies do not fill it. Galaxy spectral energy distributions, when scaled to a fixed broadband luminosity, appear to occupy a thin, onedimensional locus in color space or spectrum space (e.g., Connolly \& Szalay 1999). Spiral galaxies show a good correlation between rotation velocity and luminosity (e.g., Tully \& Fisher 1977; Giovanelli et al. 1997). Galaxy morphology is strongly correlated with broadband colors, strengths of spectral features, and inferred star formation histories (e.g., Roberts \& Haynes 1994).

Among all galaxy families, early-type (elliptical and S0) galaxies show the most precise regularities (Djorgovski \& Davis 1987; Burstein et al. 1997). Early-type galaxy surface brightness distributions follow a very simple, universal "de Vaucouleurs" profile (de Vaucouleurs 1948). Their spectral energy distributions appear to be virtually universal, showing very little variation with mass, environment, or cosmic time (e.g., van Dokkum \& Franx 1996; Pahre 1998). What variations they do show are measurable and precise. Earlytype galaxy colors, luminosities, half-light radii, velocity 
dispersions, and surface brightnesses are all correlated (Baum 1959; Fish 1964; Faber \& Jackson 1976; Kormendy 1977; Bingelli, Sandage, \& Tarenghi 1984); they can be combined into a two-dimensional "fundamental plane" with very little scatter (e.g., Dressler et al. 1987; Djorgovski \& Davis 1987; Faber et al. 1987).

The homogeneity of the early-type galaxy population is difficult to understand if early-type galaxies are assembled at late times by stochastic mergers of less massive galaxies of, presumably, different ages, star formation histories, and gas contents, as many models postulate (e.g., Larson 1975; White \& Rees 1978; van Albada 1982; Kauffmann 1996; Kauffmann \& Charlot 1998). It is possible that the homogeneity of early-type galaxies points to early formation (e.g., Worthey 1994; Bressan, Chiosi, \& Fagotto 1994; Vazdekis et al. 1996; Tantalo, Chiosi, \& Bressan 1998); certainly their stellar populations appear old (e.g., Bernardi et al. 1998; Colless et al. 1999; Trager et al. 2000a, 2000b; Kuntschner et al. 2001). Alternatively, the observable properties of the stellar content of early-type galaxies are fixed entirely by the properties of the collisionless, self-gravitating, dark matter halos in which we believe such galaxies lie (e.g., Hernquist 1990). These halos, almost by definition, are not subject to the vagaries of gas dynamics, star formation, and magnetic fields; they are influenced only by gravity.

It is essentially a stated goal of the Sloan Digital Sky Survey (SDSS; York et al. 2000; Stoughton et al. 2002) to revolutionize the study of galaxies. The SDSS is imaging $\pi$ sr of the sky (northern Galactic cap) in five bands and taking spectra of $\sim 10^{6}$ galaxies and $\sim 10^{5}$ QSOs. Among the $10^{6}$ SDSS spectra, there will be roughly $2 \times 10^{5}$ spectra taken of early-type galaxies; in fact $10^{5}$ of the spectroscopic fibers are being used to assemble a sample of luminous early-type galaxies with a larger mean redshift than the main SDSS sample (Eisenstein et al. 2001). The high quality of the SDSS five-band CCD imaging (Gunn et al. 1998; Lupton et al. 2001) allows secure identification of early-type galaxies and precise measurements of their photometric properties; most spectroscopic targets in the SDSS are detected in the imaging at signal-to-noise ratios $\mathrm{S} / \mathrm{N}>100$.

Early-type galaxy studies in the past, for technical reasons, have concentrated on galaxies in clusters at low (e.g., Jørgensen, Franx, \& Kjærgaard 1996; Ellis et al. 1997; Pahre, Djorgovski \& de Carvalho 1998b; Pahre, de Carvalho, \& Djorgovski 1998a; Scodeggio et al. 1998; Colless et al. 2001; Saglia et al. 2001; Kuntschner et al. 2001; Bernardi et al. 2002a, 2002b) and intermediate redshifts (e.g., van Dokkum et al. 1998, 2001; Kelson et al. 2000; Ziegler et al. 2001). Only the large area "Seven Samurai" (e.g., Faber et al. 1989) and ENEAR surveys (e.g., da Costa et al. 2000) of nearby early types, recent work with galaxies in the SBF survey (Blakeslee et al. 2001), and some studies at intermediate redshifts by Schade et al. (1999), Treu et al. (1999, 2001a, 2001b), and van Dokkum et al. (2001) are not restricted to cluster environments. In contrast, the SDSS is surveying a huge volume of the local universe, so the sample includes early-type galaxies in every environment from voids to groups to rich clusters. As of writing, when only a small fraction of the planned SDSS imaging and spectroscopy has been taken, the number ( $\sim 9000)$ of early-type galaxies with well-measured velocity dispersions and surface brightness profiles in the SDSS greatly exceeds the total number in the entire astronomical literature to date.
This is the first of four papers in which we use the SDSS sample to measure the fundamental plane and other earlytype galaxy correlations in multiple bands. This first paper describes how the sample was selected and presents the data. Section 2 describes the main properties of the SDSS database. The criteria used to select the early-type galaxy sample are described in $\S 3$. This section also presents a selection of images from a range of redshifts, shows de Vaucouleurs' profile fits to the observed surface brightness profiles, and argues that the SDSS photometric pipeline treatment of seeing and sky subtraction have not strongly compromised the estimates of the best-fitting model parameters. It also displays examples of the spectra from which we estimate velocity dispersions. Section 4 presents the final catalog of photometric and spectroscopic parameters, which we use for our subsequent analyses of early-type galaxy properties, and discusses some properties of the sample. Many details are relegated to the Appendices. Appendix A contains a discussion of the various $K$-corrections we have tried. The way we estimate velocity dispersions is presented in Appendix B. A novel method for estimating aperture corrections to the velocity dispersions is discussed in Appendix C. Finally, the covariance matrix of the errors is discussed in Appendix D.

Paper II (Bernardi et al. 2003a) studies correlations between various pairs of observables, such as the FaberJackson and Kormendy relations. It also presents the luminosity function and its evolution. The fundamental plane and its dependence on wave band, color, redshift, and environment is studied in Paper III (Bernardi et al. 2003b). The co-added spectra of these galaxies are studied in Paper IV (Bernardi et al. 2003c). One of the results of that paper is a library of co-added spectra that contains spectra that represent a wide range of early-type galaxies. This library is available electronically. These spectra indicate that the chemical composition of the early-type galaxy population evolves with redshift. The chemical abundances and evolution are then combined with stellar population models to estimate the ages and metallicities of the galaxies in our sample. This paper also analyzes correlations with color (e.g., colormagnitude and color- $\sigma$ relations) and discusses the effects of color gradients on measurements of the strength of the correlation between color and magnitude.

Except where stated otherwise, we write the Hubble constant as $H_{0}=100 \mathrm{hm} \mathrm{s}^{-1} \mathrm{Mpc}^{-1}$, and we perform our analysis in a cosmological world model with $\left(\Omega_{M}, \Omega_{\Lambda}\right.$, $h)=(0.3,0.7,0.7)$, where $\Omega_{M}$ and $\Omega_{\Lambda}$ are the present-day scaled densities of matter and cosmological constant. In such a model, the age of the universe at the present time is $t_{0}=9.43 h^{-1}$ Gyr. For comparison, an Einstein-de Sitter model has $\left(\Omega_{M}, \Omega_{\Lambda}\right)=(1,0)$ and $t_{0}=6.52 h^{-1}$ Gyr. We frequently use the notation $h_{70}$ as a reminder that we have set $h=0.7$. In addition, we will frequently be interested in the logarithms of physical quantities. Our convention is to set $R \equiv \log R_{o}$ and $V \equiv \log \sigma$, where $R_{o}$ and $\sigma$ are effective radii in $h_{70}^{-1} \mathrm{kpc}$ and velocity dispersions in $\mathrm{km} \mathrm{s}^{-1}$, respectively.

\section{THE SDSS DATABASE}

The SDSS project is described in Stoughton et al. (2002). The data we analyze in this paper were selected from the SDSS database in the summer of 2001. At that time, the SDSS had imaged $\sim 1500 \mathrm{deg}^{2} ; \sim 65,000$ galaxies and $\sim 8000$ QSOs had both photometric and spectroscopic information. The photometric and spectroscopic data were taken 
with the $2.5 \mathrm{~m}$ SDSS telescope at the Apache Point Observatory (New Mexico) between 1999 March and 2000 October. Details of the photometric and spectroscopic observations and data reduction procedure will be presented elsewhere. Here we briefly summarize.

\subsection{SDSS Imaging Data}

Images are obtained by drift-scanning with a mosaic CCD camera (Gunn et al. 1998) that gives a field of view of $3 \times 3 \mathrm{deg}^{2}$, with a spatial scale of 0 ". 4 pixel $^{-1}$ in five bandpasses $(u, g, r, i$, and $z)$ with central wavelengths $(3560,4680$, 6180,7500 , and $8870 \AA$ A; Fukugita et al. 1996). The errors in $u$-band measurements are larger than the others, so we will only present results in the other four bands. In addition, the photometric solutions we use in this paper are preliminary (for details, see discussion of the Early Data Release in Stoughton et al. 2002); we use $r^{*}$ rather than $r$, and similarly for the other bands, to denote this.

The effective integration time is $54 \mathrm{~s}$. The raw CCD images are bias-subtracted, flat-fielded, and backgroundsubtracted. Pixels contaminated by the light of cosmic rays and bad columns are masked. Astronomical sources are detected, and overlapping sources are deblended. The data are flux-calibrated by comparison with a set of overlapping standard-star fields calibrated with a $0.5 \mathrm{~m}$ "Photometric Telescope" (Hogg et al. 2001; Smith et al. 2002). The Photometric Telescope is also used for measuring the atmospheric extinction coefficients in the five bands. The median effective seeing (the median FWHM of the stellar profiles) for the observations used here is 1 1" 5 . All of this image processing is performed with software specially designed for reducing SDSS data (Lupton et al. 2001). The uncertainty in the $r^{*}$-band zero-point calibration is less than $0.01 \mathrm{mag}$; the uncertainty in the sky background subtraction is less than about $1 \%$.

Surface photometry measurements are obtained by fitting a set of two-dimensional models to the images. The model fits account for the effects of seeing, atmospheric extinction, and Galactic extinction (this last one uses the results of Schlegel, Finkbeiner, \& Davis 1998). The SDSS model for seeing is described in Stoughton et al. (2002). Briefly, the point-spread function (PSF) is expected to have a Fourier transform, $\propto \exp \left[-\left(k r_{\mathrm{PSF}} / 2\right)^{5 / 3}\right]$, characteristic of Kolmogorov turbulence (e.g., Saglia et al. 1993). This PSF is approximated as the sum of Gaussians (this parametrization allows for a substantial reduction in the processing time required to deconvolve the effects of seeing).

The SDSS photometric pipeline fits two models to the two-dimensional image of each object in each band: a pure de Vaucouleurs' profile and a pure exponential profile (e.g., Stoughton et al. 2002). Briefly, the photon counts are binned into a number of radial bins and 12 angular bins. Then, two-dimensional de Vaucouleurs' and exponential models, convolved with the seeing, are fitted to the cumulative binned counts. The pipeline does not include more complicated models (e.g., bulge plus disk) because the computational expense to compute them is not justified for the majority of the detected objects. Algorithms that fit bulge and disk components (which are desirable to fit on large galaxies) to the surface brightness profiles are currently being developed within the collaboration, but are not yet available. We present examples of $r^{*}$-band images and mean surface brightness profiles in $\S 3.2 .2$.
The SDSS image processing software provides several global photometric parameters, for each object, which are obtained independently in each of the five bands. Because we are interested in early-type galaxies, we use primarily the following:

1. The ratio $b / a$ of the lengths of the minor and major axes of the observed surface brightness profile.

2. The effective radius (or half-light radius) $r_{\mathrm{dev}}$ along the major axis and the total magnitude $m_{\mathrm{dev}}$; these are computed by fitting a two-dimensional version of de Vaucouleurs (1948) $r^{1 / 4}$ model to the observed surface brightness profile. (The fitting procedure accounts for the effects of seeing - we test the accuracy of this procedure below.)

3. Likelihood parameters deVL and expL that indicate how well the de Vaucouleurs' or exponential models, when convolved with a model for the seeing, fit the observed light profile.

4. The model magnitude $m_{m}$; this is the total magnitude calculated by using the (de Vaucouleurs' or exponential) model that fits the galaxy profile best in the $r^{*}$ band. The model magnitudes in the other four bands are computed using that $r^{*}$ fit as a filter; in effect, this measures the colors of a galaxy through the same aperture.

5. The Petrosian magnitude $m_{\mathrm{P}}$ is also computed; this is the flux within $2 r_{\mathrm{P}}$, where $r_{\mathrm{P}}$ is defined as the angular radius at which the ratio of the local surface brightness at $r$ to the mean surface brightness within a radius $r$ is 0.2 (Petrosian 1976).

6. The Petrosian radii $r_{50}$ and $r_{90}$; these are the angular radii containing $50 \%$ and $90 \%$ of the Petrosian light, respectively.

Although our analyses on early-type galaxy properties presented here and in the companion papers have been performed with both the de Vaucouleurs' fit parameters and the Petrosian quantities, in most of our analyses only the results of the de Vaucouleurs' fits are presented. This is because the de Vaucouleurs' model appears to be a very good fit to the early-type galaxy surface brightness profiles in the SDSS sample and because it is conventional, in the literature on early-type galaxies, to use these quantities. On the other hand, for reasons given in Stoughton et al. (2002), unless stated otherwise, galaxy colors are always computed using model magnitudes.

\subsection{SDSS Spectroscopic Data}

The SDSS takes spectra only for a target subsample of objects. Target selection criteria are described in Stoughton et al. (2002) and Strauss et al. (2002). Spectra are obtained using a multiobject spectrograph that observes 640 objects at once. Each spectroscopic plug plate, 1.5 in radius, has 640 fibers, each $3^{\prime \prime}$ in diameter. Two fibers cannot be closer than $55^{\prime \prime}$ due to the physical size of the fiber plug. Typically $\sim 500$ fibers per plate are used for galaxies, $\sim 90$ for QSOs, and the remaining for sky spectra and spectrophotometric standard stars.

Each plate typically has three to five spectroscopic exposures of 15 minutes, depending on the observing conditions (weather, moon); a minimum of three exposures is taken to ensure adequate cosmic-ray rejection. For galaxies at $z \leq 0.3$, the median spectrum S/N per pixel is 16 (see Fig. 18 in Appendix B). The wavelength range of each spectrum is 3900-9000 А. The instrumental dispersion is $\log \lambda=10^{-4}$ 
dex pixel ${ }^{-1}$, which corresponds to $69 \mathrm{~km} \mathrm{~s}^{-1}$ per pixel. (There is actually some variation in this instrumental dispersion with wavelength, which we account for; see Fig. 16 and associated discussion in Appendix B.) The instrumental resolution of galaxy spectra, measured from the autocorrelation of stellar template spectra, ranges from 85 to $105 \mathrm{~km} \mathrm{~s}^{-1}$, with a median value of $92 \mathrm{~km} \mathrm{~s}^{-1}$.

A highly automated software package has been designed for reducing SDSS spectral data. The raw data are biassubtracted, flat-fielded, wavelength-calibrated, sky lines removed, co-added, cleaned from residual glitches (cosmic rays, bad pixels), and flux-calibrated. The spectrosoftware classifies objects by spectral type and determines emission and absorption redshifts. (Redshifts are corrected to the heliocentric reference frame.) The redshift success rate for objects targeted as galaxies is greater than $99 \%$, and errors in the measured redshift are less than about $10^{-4}$. Once the redshift has been determined, the following quantities are computed: Absorption line strengths (Brodie \& Hanes 1986; Diaz, Terlevich, \& Terlevich 1989; Trager et al. 1998), equivalent widths of the emission lines, and eigencoefficients and classification numbers of a principal component analysis (PCA; Connolly \& Szalay 1999). Some information about the reliability of the redshift and the quality of the spectrum is also provided. We present examples of the spectra in $\S 3.3 .2$.

The SDSS pipeline does not provide an estimate of the line-of-sight velocity dispersion, $\sigma$, within a galaxy, so we compute it separately for the early-type galaxy sample (see $\S 3.3 .1)$.

\section{THE SAMPLE}

\subsection{Selection Criteria}

The main goal of this series of papers is to study the properties of early-type galaxies using the main galaxy sample of the SDSS database. Therefore, one of the crucial steps in our study is the separation of galaxies into early and late types. We want to select objects whose spectra are good enough to compute the central velocity dispersion. In addition, because we wish to study the colors of the galaxies in our sample, we must not use color information to select the sample. To reach our goal, we have selected galaxies that satisfy the following criteria:

1. Concentration index $r_{90} / r_{50}>2.5$ in $i^{*}$;

2. The likelihood of the de Vaucouleurs' model is at least 1.03 times the likelihood of the exponential model;

3. Spectra with PCA classification numbers $a<-0.1$, typical of early-type galaxy spectra (Connolly \& Szalay 1999);

4. Spectra without masked regions (the SDSS spectroscopic pipeline outputs a warning flag for spectra of low quality; we only chose spectra for which this flag was set to zero);

5. $\mathrm{S} / \mathrm{N}>10$

6. Redshift less than 0.3 .

Section 3.3.1 describes how we estimate the velocity dispersion $\sigma$ for galaxies that satisfy the above criteria. We consider velocity dispersion estimates smaller than about $70 \mathrm{~km}$ $\mathrm{s}^{-1}$ to be unreliable (see Appendix B). Therefore, in the final sample presented in $\S 4$, galaxies with $\sigma \leq 70 \mathrm{~km} \mathrm{~s}^{-1}$ have been excluded. The results of this and the companion papers are not significantly different if we change the cutoff on velocity dispersions to $100 \mathrm{~km} \mathrm{~s}^{-1}$. About 9,000 objects satisfied all the above criteria.

As stated earlier, the SDSS pipeline does not output diskto-bulge ratios from fits to the light profiles. The first two requirements above attempt to select profile shapes that are likely to be those of spheroidal systems. The $i^{*}$-band measurements tend to be less noisy than $g^{*}$ or $z^{*}$, so we chose to use the $i^{*}$-band estimate of how centrally concentrated the light is. The second requirement reduces approximately to requiring that $\mathrm{deVL} /(\mathrm{deVL}+\operatorname{expL})>0.5$; that is, the surface brightness profile should be better fitted by the de Vaucouleurs' model than by an exponential.

The spectra of late-type galaxies show emission lines, so examining the spectra (e.g., using the PCA classification) is a simple way of removing such objects from the sample. Because the aperture of an SDSS spectroscopic fiber $\left(3^{\prime \prime}\right)$ samples only the inner parts of nearby galaxies, and because the spectrum of the bulge of a nearby late-type galaxy can resemble that of an early-type galaxy, it is possible that some nearby late-type galaxies could be mistakenly included in the sample (e.g., Kochanek, Pahre, \& Falco 2000). Most of these will have been excluded by the first two cuts on the shape of the light profile. To check this, we visually inspected all galaxies with $r_{\mathrm{dev}}>8^{\prime \prime}$. About $\sim 50$ of 225 (i.e., about $20 \%$ ) looked like late-types, and so we removed them. Note that weak emission lines, such as $\mathrm{H} \alpha$ and/or $\mathrm{O}$ II, are still present in the early-type galaxy spectra in our sample. For example, the median $\mathrm{H} \alpha$ equivalent width for galaxies in our sample is $-1.4 \AA$, but $\sim 5 \%$ of the galaxies have equivalent widths larger than $1 \AA$, and $\sim 10 \%$ of these (i.e., $0.5 \%$ of the sample) have equivalent widths in the range 4-10 $\mathrm{A}$. Similarly, although the median equivalent width of $\mathrm{O}$ II is $1.5 \AA, \sim 15 \%$ of the galaxies show $\mathrm{O}$ II equivalent widths larger than $4 \AA$, and $\sim 10 \%$ of these (i.e., $1.5 \%$ of the sample) have equivalent widths ranging from 8 to $15 \AA$.

The reason for our redshift cut is as follows. The SDSS main galaxy sample from which we select our early-type galaxies is apparent magnitude limited in $r^{*}$, where the limits are defined using Petrosian magnitudes $m_{\mathrm{P}}$. This means that the main galaxy sample is not magnitude-limited in the other bands. Blanton et al. (2001) describe the cuts in $m_{\mathrm{P}}$ one must apply in the other bands to obtain complete magnitudelimited samples. They also note that $m_{\mathrm{dev}} \approx 2.5 \log 0.8+$ $m_{\mathrm{P}}$. Since we will almost always be working with $m_{\mathrm{dev}}$ rather than $m_{\mathrm{P}}$, our cuts are slightly different from theirs; the cuts we use to define magnitude-limited samples in the different bands are summarized in Table 1 (also see Fig. 2).

At $z \geq 0.3$, however, most early-type galaxies in the SDSS database were targeted using different selection criteria than were used for the main SDSS galaxy sample (see Strauss et al. 2002; they make up the Luminous Red Galaxy sample described by Eisenstein et al. 2001). In the interest of keeping our sample as close to being magnitude-limited as possible, we restricted our sample to $z \leq 0.3$. In addition, one might expect an increasing fraction of the early-type population at higher redshifts to have emission lines: if so, then our removal of emission-line objects amounts to a small but redshift-dependent selection effect. Since our sample is restricted to $z \leq 0.3$, this bias should be small.

Before moving on, it is worth pointing out that there is a morphologically based selection cut that we could have made but did not. Elliptical galaxies are expected to have axis ratios greater than about 0.6 (e.g., Binney \& Tremaine 1987). Since we have axis ratio measurements of all the 
TABLE 1

Photometric Parameters and Median Errors of the Objects in Our Sample

\begin{tabular}{|c|c|c|c|c|c|c|c|c|c|}
\hline Band & $\begin{array}{c}m_{\min } \\
(\mathrm{mag})\end{array}$ & $\begin{array}{l}m_{\max } \\
(\mathrm{mag})\end{array}$ & $\begin{array}{l}\delta m_{\mathrm{dev}} \\
(\mathrm{mag})\end{array}$ & $\begin{array}{c}\delta \log r_{o} \\
(\operatorname{dex})\end{array}$ & $\begin{array}{c}\delta \log I_{o} \\
(\operatorname{dex})\end{array}$ & $\begin{array}{c}\delta m_{m} \\
(\mathrm{mag})\end{array}$ & $\begin{array}{l}\delta m_{\text {pet }} \\
(\mathrm{mag})\end{array}$ & $\begin{array}{c}\delta \log r_{50} \\
(\mathrm{dex})\end{array}$ & $\begin{array}{c}\delta \log r_{90} \\
(\mathrm{dex})\end{array}$ \\
\hline$g^{*} \ldots \ldots \ldots \ldots \ldots \ldots \ldots$ & 15.50 & 18.10 & 0.03 & 0.02 & 0.04 & 0.03 & 0.04 & 0.02 & 0.02 \\
\hline$r^{*} \ldots \ldots \ldots \ldots \ldots \ldots$ & 14.50 & 17.45 & 0.02 & 0.01 & 0.03 & 0.02 & 0.02 & 0.01 & 0.01 \\
\hline .............. & 14.50 & 17.00 & 0.02 & 0.01 & 0.03 & 0.02 & 0.02 & 0.01 & 0.01 \\
\hline$z^{*} \ldots \ldots \ldots \ldots \ldots \ldots$ & 14.50 & 16.70 & 0.05 & 0.03 & 0.09 & 0.05 & 0.05 & 0.03 & 0.03 \\
\hline
\end{tabular}

objects in our sample, we could have included a cut on $b / a$. The bottom right panel of Figure 1 shows the distribution axis ratios $b / a$ in our $r^{*}$ sample: about $20 \%$ of the objects in it have $b / a \leq 0.6$. (The spike at $b / a=1$ is artificial - the pipeline reports large uncertainties in the fitted value of $b / a$ for these objects.)

Our combination of cuts on the shapes of the light profiles and spectral features mean that these objects are unlikely to be late-type galaxies. Indeed, a visual inspection of a random sample of the objects with axis ratios smaller than 0.6 shows that they look like S0's. The bottom left panel of Figure 1 shows that $b / a$ does not correlate with color: in particular, the colors of the most flattened objects are not bluer than in the rest of the sample. In addition, recall that all objects with $r_{\mathrm{dev}} \geq 8^{\prime \prime}$ were visually inspected, and these, despite having $b / a \leq 0.6$ (top left), did not appear peculiar. In addition, $b / a$ does not correlate with surface brightness or apparent magnitude. Galaxies with small angular sizes $r_{\mathrm{dev}}$ are assigned large values of $b / a$ only slightly more often than average (top left; the median $b / a$ is $0.79,0.78,0.76$, and 0.7 for $r_{\mathrm{dev}}$ in the range $1^{\prime \prime}-2^{\prime \prime}, 2^{\prime \prime}-3^{\prime \prime}, 3^{\prime \prime}-4^{\prime \prime}$, and greater than $\left.4^{\prime \prime}\right)$. However, there is a weak trend for the objects at higher $z$ to be rounder (middle right). This trend with $z$ may be related to the magnitude limit of our sample rather than reflecting problems associated with the fits to the observed light profiles. (The two bands at $z \sim 0.08$ and 0.13 show that the effects of large-scale clustering in our sample are significant.) Section 3.2.1 describes how we convert from the observed half-light radius $r_{\mathrm{dev}}$ to an estimate of the physical half-light radius $R_{o}$, and $\S 3.3 .1$ discusses how we estimate velocity dispersions. Objects with smaller values of $R_{O}$ tend to be more flattened (top right) and to have slightly smaller velocity dispersions (middle left); because of the magnitude limit, these objects drop out of our sample at higher

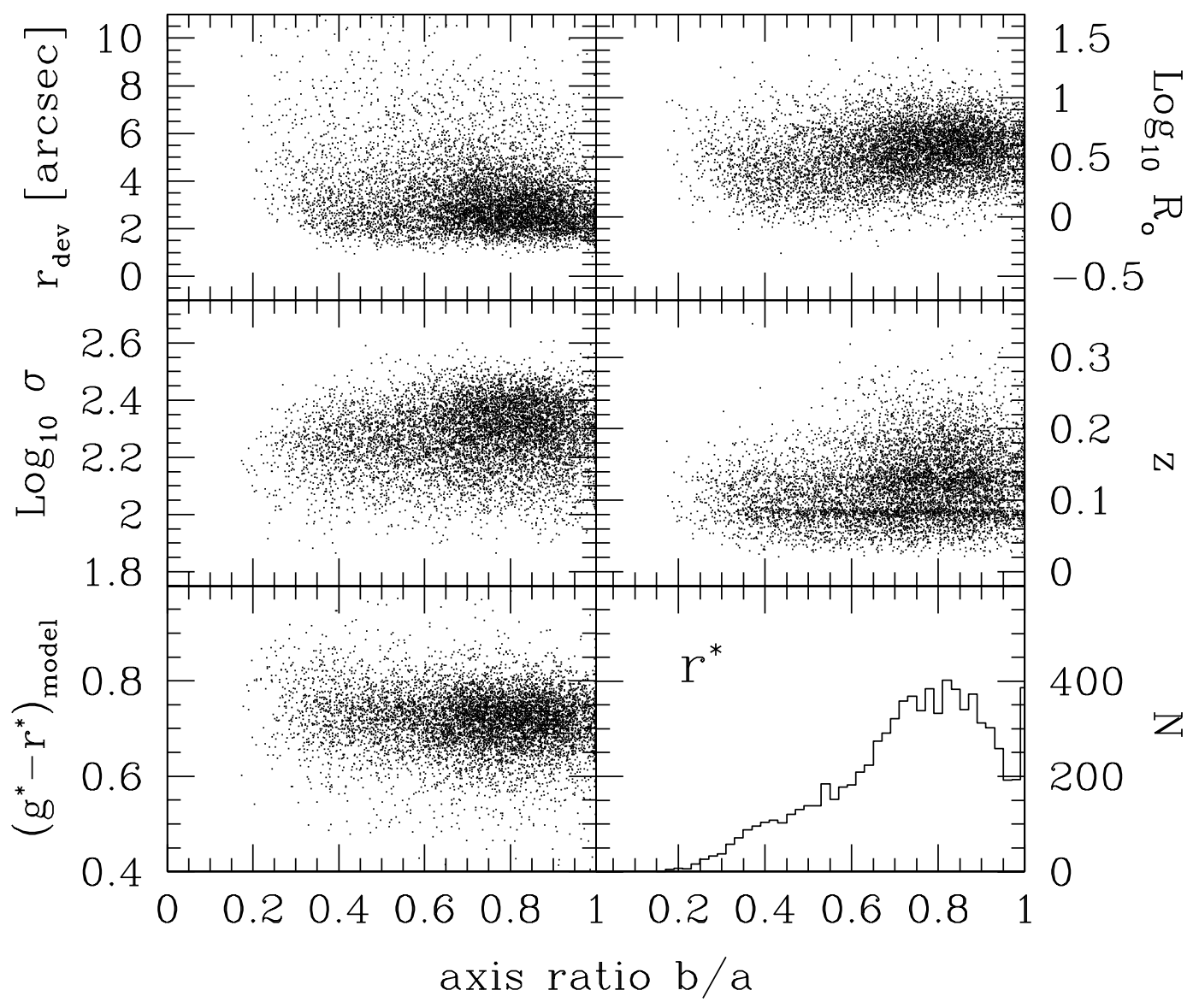

FIG. 1.-Effective angular sizes $r_{\mathrm{dev}}$, effective circular physical sizes $R_{o}$, velocity dispersions $\sigma$, redshifts $z$, and $g^{*}-r^{*}$ colors as a function of axis ratio $b / a$ for the galaxies in our $r^{*}$ sample. Bottom right panel shows that the typical axis ratio is $b / a \approx 0.8$. There is only a weak tendency for galaxies with small $r_{\text {dev }}$ to be rounder, suggesting that the estimate of the shape is not compromised by seeing (typical seeing is about 1".5). Results in the other bands are similar. 
redshifts. Requiring that $b / a \geq 0.6$ would remove such objects from our sample completely.

In Paper III, we study the fundamental plane populated by the galaxies in this sample. Excluding all objects with $b / a<0.6$ has no effect on the shape of this plane. As a result, in the interests of keeping our sample as close to being magnitude-limited as possible, we chose not to make an additional selection cut on $b / a$.

\subsection{Photometric Data of the Sample}

\subsubsection{Conversion to Rest-Frame Luminosities and Sizes}

Figure 2 shows the distribution of the $m_{\mathrm{dev}}$ apparent magnitudes in our sample as a function of redshift. The dashed lines in each panel show the magnitude limits summarized in Table 1; the magnitude-limited samples span the redshift range $0.01 \leq z \leq 0.3$.

To convert the apparent magnitude $m$ to an absolute magnitude $M$, we must assume a particular cosmology and account for the fact that at different redshifts an observed bandpass corresponds to different rest-frame bands (the $K$ correction). We write the Hubble constant today as $100 h$ $\mathrm{km} \mathrm{s}^{-1} \mathrm{Mpc}^{-1}$ and use $\left(\Omega_{M}, \Omega_{\Lambda}, h\right)=(0.3,0.7,0.7)$. Most of our sample is at $c z \geq 9000 \mathrm{~km} \mathrm{~s}^{-1}$; since line-of-sight peculiar velocities are not expected to exceed more than a few thousand kilometers per second, we feel that it is reasonable to assume that all of a galaxy's redshift is due to the Hubble recession velocity. This means that we can compute the absolute magnitude in a given band by $M=m-5 \log \left[D_{L}(z\right.$; $\left.\left.\Omega_{M} ; \Omega_{\Lambda}\right)\right]-25-K(z)$, where $m$ is the apparent magnitude, $D_{L}$ is the luminosity distance in megaparsecs (from, e.g.,
Weinberg 1972; Hogg 1999), and $K(z)$ is the $K$-correction for the band.

Because we have five colors and a spectrum for each galaxy, we could, in principle, compute an empirical $K$-correction for each galaxy. This requires a good understanding of the accuracy of the SDSS photometry and spectroscopy and should be possible when the survey is closer to completion. Rather than follow the procedure adopted by the 2dFGRS (Madgwick et al. 2002) or a procedure based on finding the closest template spectrum to each galaxy and using it to compute the $K$-correction (e.g., Lin et al. 1999 for the CNOC2 survey), we use a single redshift-dependent template spectrum to estimate the $K$-correction. In effect, although this allows galaxies at different redshift to be different, it ignores the fact that not all galaxies at the same redshift are alike. As a result, the absolute luminosities we compute are not as accurate as they could be, and this can introduce scatter in the various correlations we study below. Of course, using a realistic $K$-correction is important, because inaccuracies in $K(z)$ can masquerade as evolutionary trends.

For the reasons discussed more fully in Appendix A, our $K$-corrections are based on a combination of Bruzual \& Charlot (2003) and Coleman, Wu, \& Weedman (1980) prescriptions. Specifically, the $K$-corrections we apply were obtained by taking a Bruzual \& Charlot model for a $10^{11}$ $M_{\odot}$ object that formed its stars with an initial mass function (IMF) given by Kroupa (2001) in a single solar metallicity and abundance ratio burst 9 Gyr ago, computing the difference between the $K$-correction when evolution is allowed and ignored, and adding this difference to the $K$-corrections

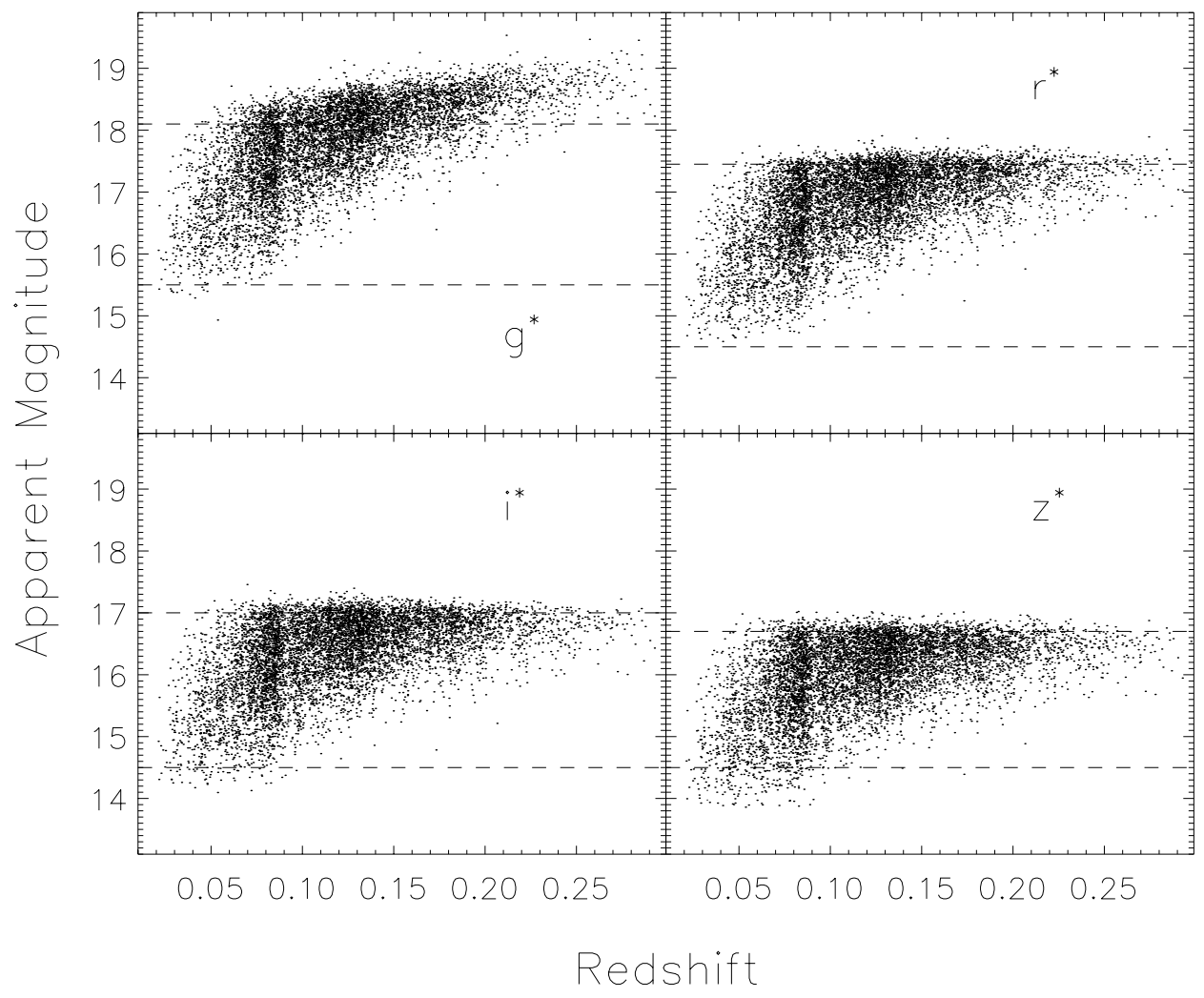

FIG. 2.-Apparent magnitude ( $\left.m_{\mathrm{dev}}\right)$ in the $g^{*}, r^{*}, i^{*}$, and $z^{*}$ bands vs. redshift for galaxies that satisfied our selection criteria. In each band, magnitudelimited samples are defined by including only those galaxies that fall between the dashed lines shown; these magnitude limits are summarized in Table 1 . 
associated with a Coleman et al. early-type galaxy template. The results that follow are qualitatively similar for a number of other $K$-correction schemes (see Appendix A for details).

In addition to correcting the observed apparent magnitudes to absolute magnitudes, we must also apply two corrections to convert the (seeing-corrected) effective angular radii, $r_{\mathrm{dev}}$, output by the SDSS pipeline to physical radii. First, we define the equivalent circular effective radius $r_{o} \equiv(b / a) r_{\mathrm{dev}}$. (Although the convention is to use $r_{e}$ to denote the effective radius, we feel that the notation $r_{o}$ is better, since it emphasizes that the radius is an effective circular, rather than elliptical aperture.) The reason we must make a second correction is shown in Figure 3. The different panels show the distribution of (seeing-corrected) effective angular sizes $r_{\mathrm{dev}}$ of the galaxies in the different bands. Note that $r_{\mathrm{dev}}$ for most of the objects is larger than the typical seeing scale of 1".5: we discuss this further in $\S 3.2 .2$. Comparison of the mean sizes in the different panels (the text in the top right corner of each panel) shows that galaxies appear slightly larger in the bluer bands.

Because our sample covers a reasonably large range in redshift, this trend means we must correct the effective sizes to a fixed rest-frame wavelength. Therefore, when converting from effective angular size $r_{o}$ to effective physical size $R_{o}$ we correct $r_{o}$ (and the Petrosian radii $r_{50}$ and $r_{90}$ ) in each band by linearly interpolating from the observed bandpasses to the central rest wavelength of each filter. The typical correction is of the order of $4 \%$, although it is sometimes as large as $10 \%$. In this respect, this correction is analogous to the $K$-correction we would ideally have applied to the magnitude and surface brightness of each galaxy. Note that the dependence of size on wavelength is also important for analyses of color gradients and the color-magnitude relation presented in Paper IV.

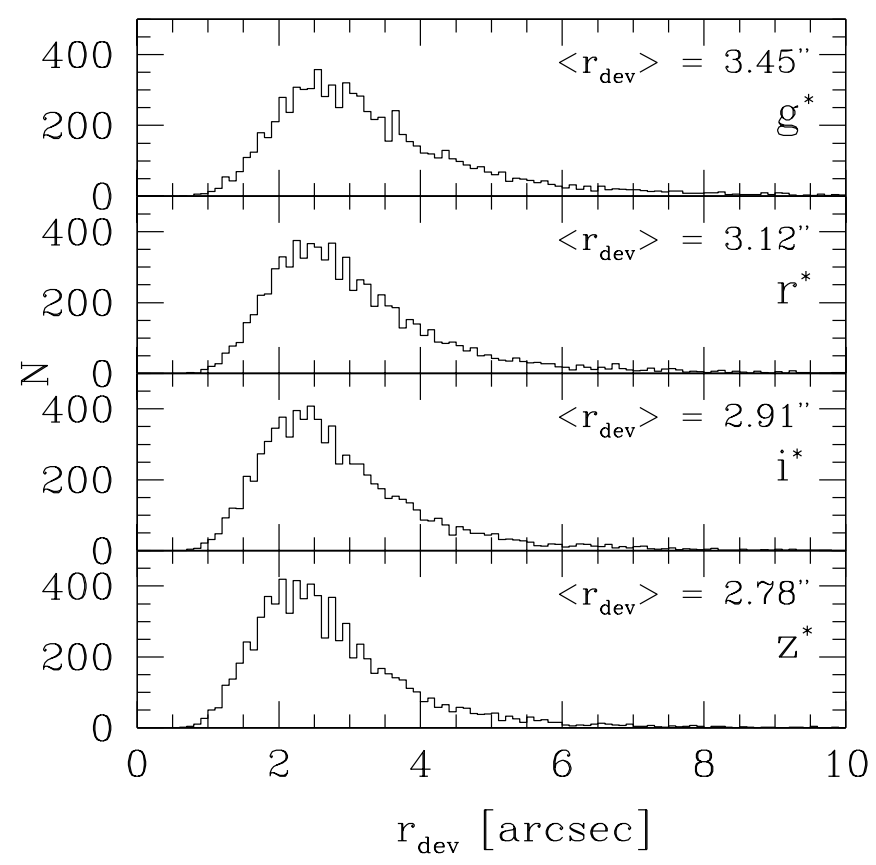

FIG. 3.-Distribution of (seeing-corrected) effective angular sizes of galaxies in our sample. Typical seeing is about 1".5. The distribution of effective radii in all the bands are very similar, although the radii are slightly larger in the bluer bands.
Our study will also require the effective surface brightness $\mu_{o} \equiv-2.5 \log I_{o}$, where $I_{o}$ is the mean surface brightness within the effective radius $R_{o}$ (as opposed to the surface brightness at $R_{o}$ ). In particular, we set $\mu_{o}=m_{\mathrm{dev}}$ $+2.5 \log \left(2 \pi r_{o}^{2}\right)-K(z)-10 \log (1+z)$. Note that this quantity is $K$-corrected and also corrected for the cosmological $(1+z)^{4}$ dimming. Our earlier remarks about the $K$ correction are also relevant here.

\subsubsection{Reliability of Photometric Parameters}

There is some discussion in the literature about the effects of seeing on estimates of the effective radius, and hence the effective surface brightness. These effects are unimportant if the seeing scale, $r_{\mathrm{PSF}}$ (the FWHM in arcseconds), is an order of magnitude smaller than the effective radius (e.g., Saglia et al. 1993, 1997). Figure 4 shows histograms of $r_{\mathrm{dev}} / r_{\mathrm{PSF}}$ in the $g^{*}, r^{*}, i^{*}$, and $z^{*}$ bands: the effective radius is typically only a factor of 2 larger than the seeing. Therefore, the accuracy of our estimates of the effective radii depend crucially on the correction for seeing being accurate.

To illustrate that the de Vaucouleurs' model fits output by the SDSS photometric pipeline are reasonable, Figure 5 shows the ratio of $\left[H_{0} d_{A}(z) / c\right]$ times the half-light radius to $r_{\mathrm{PSF}}$ in the $g^{*}$ and $r^{*}$ bands (top and bottom) as a function of redshift, in a few volume-limited catalogs. The catalog limits were chosen to be $0.5 \mathrm{mag}$ wide, so the galaxies in any given catalog should all be quite similar to each other. (The factor $H_{0} d_{A}(z) / c$ removes the expected scaling of angular diameter with redshift.) It is important to use volume-limited samples to make the measurement, because size is known to correlate with luminosity (more luminous galaxies are larger on average), and in a magnitude-limited sample such as ours, only the more luminous objects are present at higher redshifts.

In the panels on the left, the half-light radius is estimated by $r_{\mathrm{dev}}$, which has been corrected for seeing, whereas the panels on the right use $r_{50}$, which is not seeing-corrected. Seeing tends to increase the half-light radius, and indeed, in the panel on the right, there is a clear trend for the half-light radii to increase with redshift. This trend is absent in the panels on the left, suggesting that the fitting procedure accounts quite well for the effects of seeing. The panels on the left even show a slight decrease with redshift - we argue later (see discussion of Fig. 12 in $\S 4$ ) that this may be a consequence of luminosity evolution.

We have argued that our estimates of $r_{\mathrm{dev}}$ are probably not strongly compromised by seeing. In our data set, $r_{\text {dev }}$ is about a factor of 2 smaller than the radius $r_{10 \%}$ at which the fitted surface brightness is $10 \%$ of the sky level. Therefore, errors in the sky subtraction may also compromise our estimates of $r_{\mathrm{dev}}$. If the same object is moved to higher redshift but the sky level is kept fixed, then one expects $7.67\left(r_{10 \%} /\right.$ $\left.r_{\mathrm{dev}}\right)^{1 / 4}=$ const $-4 \ln (1+z)$ because cosmological surface brightness dimming scales as $(1+z)^{4}$.

Therefore, a plot of $\left(r_{10 \%} / r_{\mathrm{dev}}\right)^{1 / 4}$ versus $\ln (1+z)$, measured from a volume-limited catalog, should have a slope of $-4 / 7.67$. However, because each volume-limited catalog spans a large range in redshift, we must also account for the surface brightness dimming associated with the fact that redshift-dependent $K$-corrections must be made to estimate fixed rest-frame luminosities. Therefore, we expect the scaling with redshift to be slightly stronger: $\left(r_{10 \%} / r_{\mathrm{dev}}\right)^{1 / 4}=$ const $-0.52 \ln (1+z)-0.052 \ln (10) K(z)$. 


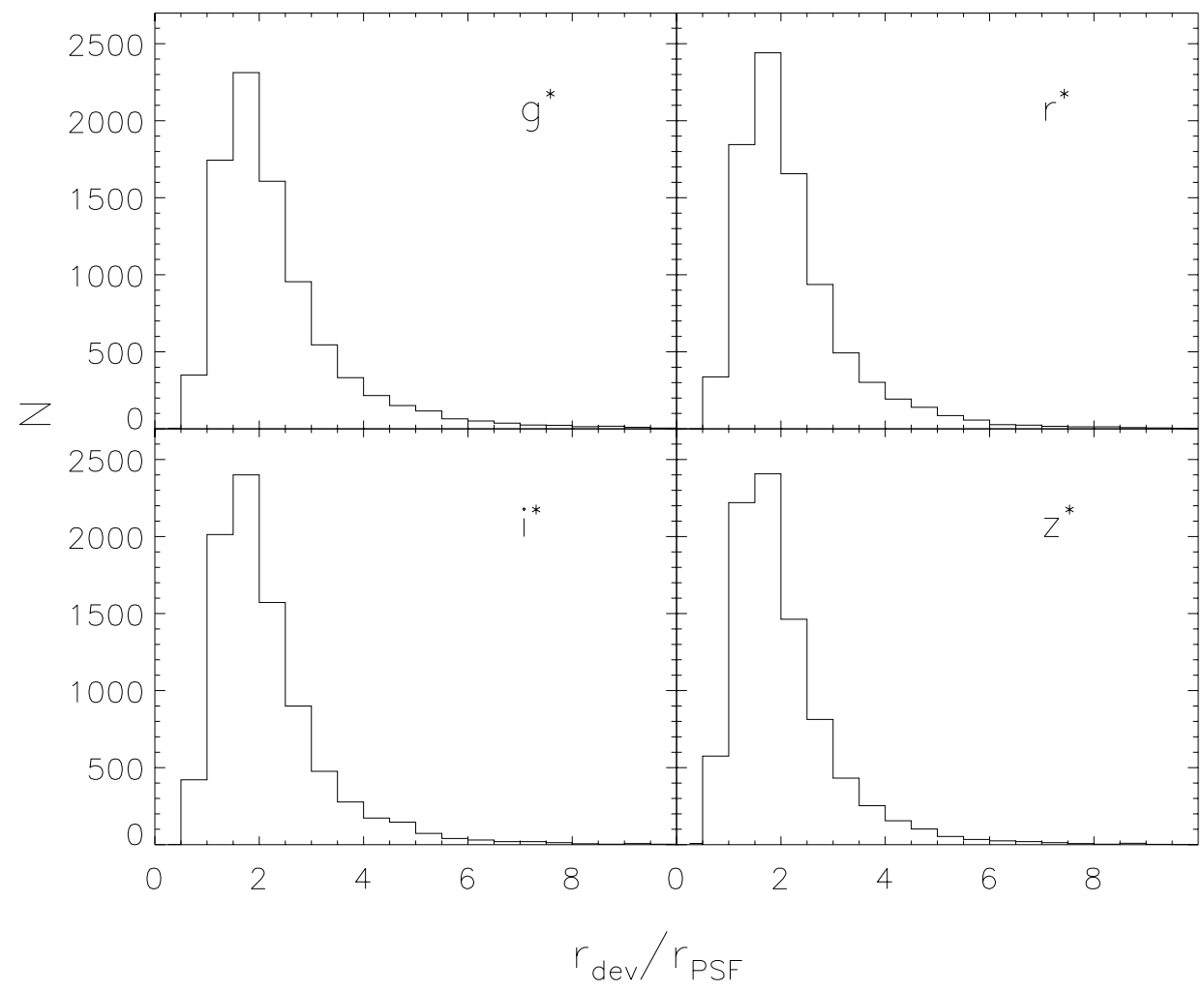

FIG. 4.-Distribution of ratio between the effective radius $r_{\mathrm{dev}}$ and $r_{\mathrm{PSF}}$, the FWHM of the PSF; the effective radius is typically about a factor of 2 larger than the seeing.

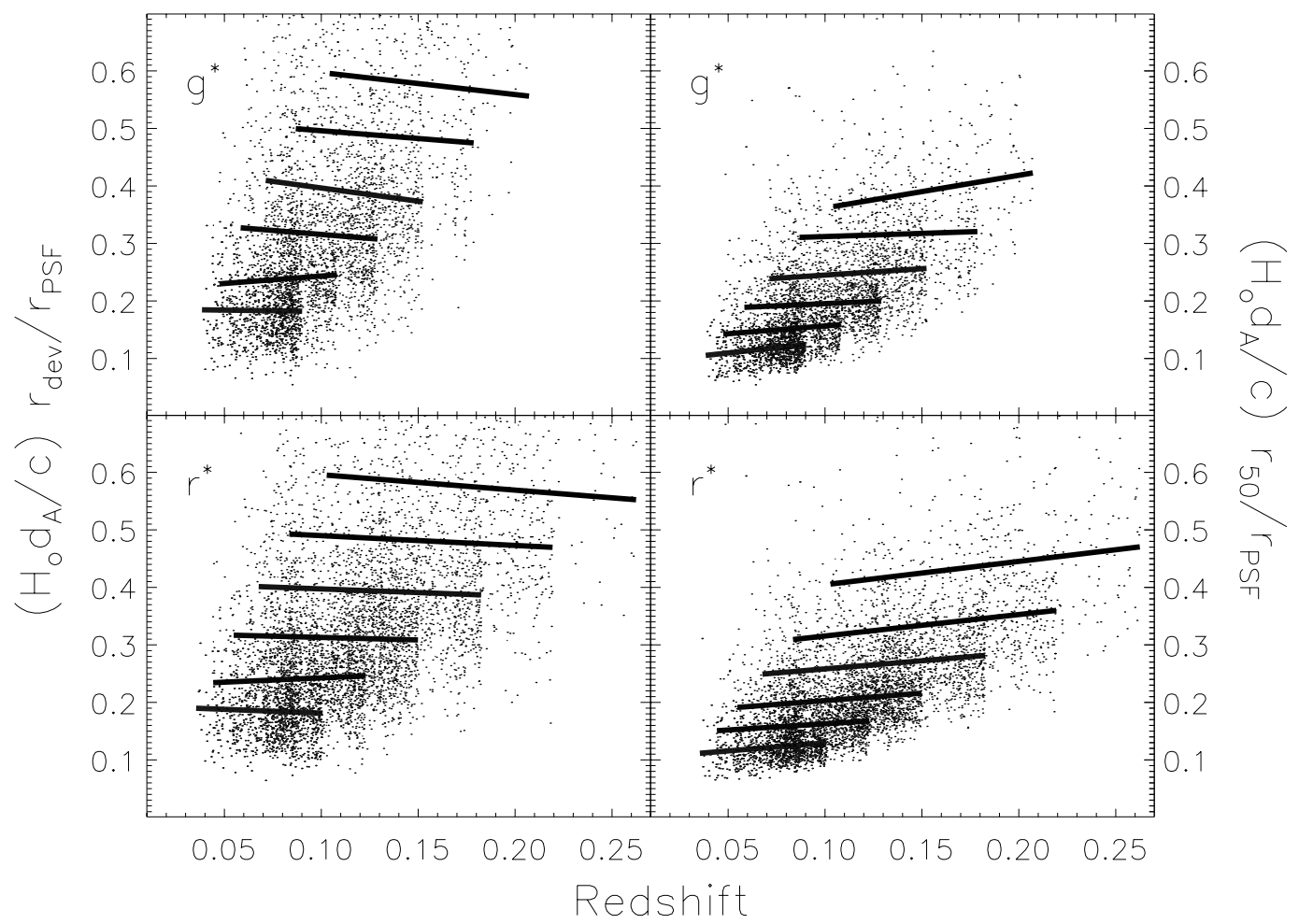

FIG. 5.-Effect of seeing on estimates of the half-light radius in the $g^{*}$ and $r^{*}$ bands (top and bottom). Each panel shows the ratio of $\left[H_{0} d_{A}(z) / c\right]$ times the angular half-light radius to $r_{\mathrm{PSF}}$ as a function of redshift in volume-limited catalogs chosen to be 0.5 mag wide. The factor $H_{0} d_{A}(z) / c$ removes the expected scaling of angular diameter with redshift. In the panels on the left, the half-light radius shown is $r_{\mathrm{dev}}$, which has been corrected for seeing, whereas the panels on the right use $r_{50}$, which is not seeing-corrected. In the panels on the right, there is a clear trend for the half-light radii to increase with redshift, suggesting that seeing is affecting the estimates. This trend is absent in the panels on the left. (The panels on the left show a slight decrease with redshift- this may be a consequence of luminosity evolution.) 


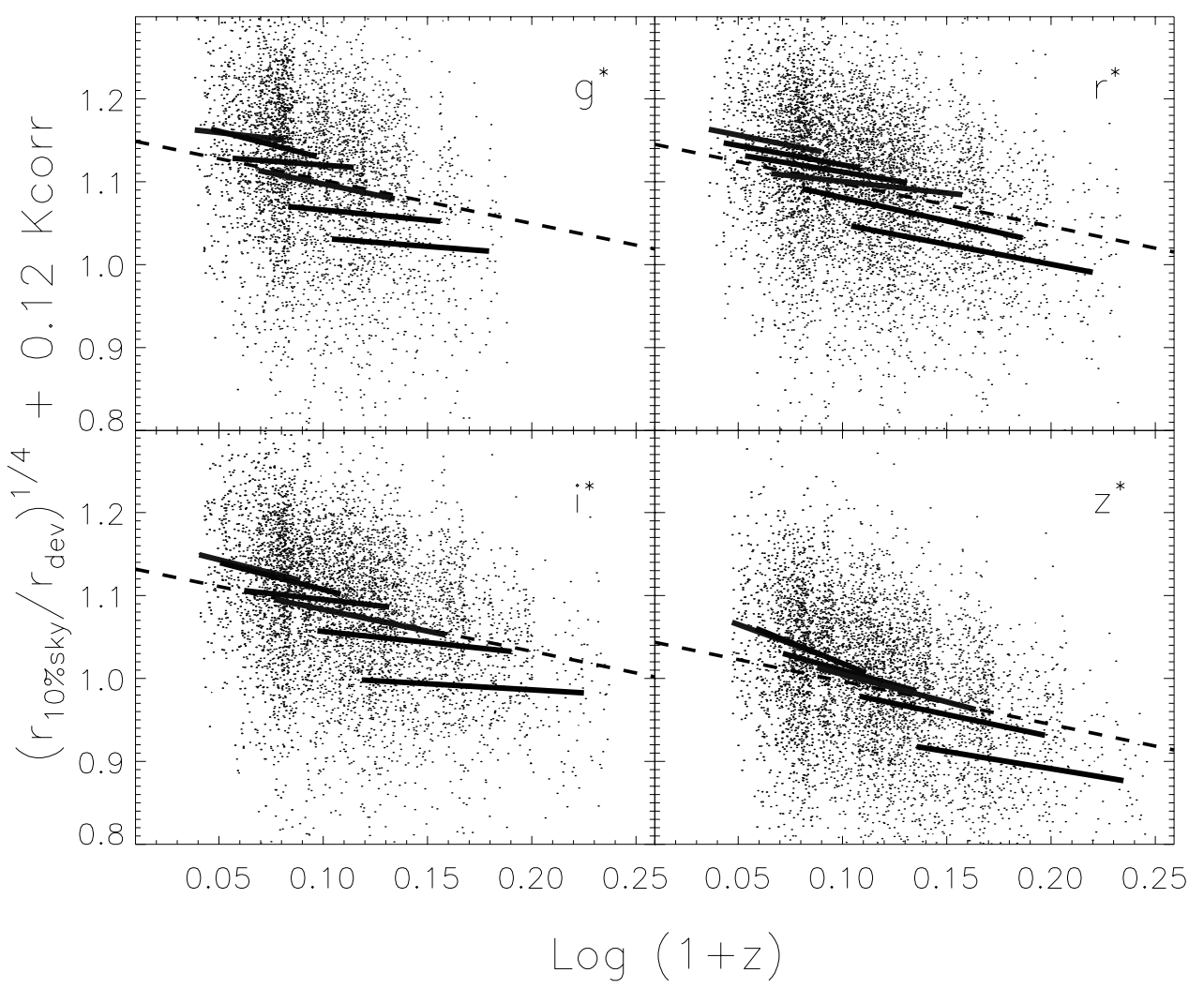

FIG. 6. - Ratio of the radius $r_{10 \% \text { sky }}$ at which the fitted surface brightness is $10 \%$ of the sky level and the effective radius $r_{\text {dev }}$, as a function of redshift, for a few volume-limited subsamples. In all four bands, this ratio decreases with increasing redshift, although the trend is slightly weaker in $g^{*}$ than in the other bands. Dashed line shows the expected scaling if there is no luminosity or size evolution. The trend for the combination of all the samples is substantially steeper than that within any one volume-limited sample, suggesting that the typical size increases strongly with luminosity.

Figure 6 shows how $\left(r_{10 \%} / r_{\mathrm{dev}}\right)^{1 / 4}+0.12 K(z)$ depends on $\ln (1+z)$ in the same volume-limited catalogs as before; solid lines show the mean trend in the faintest (top) to the most luminous (bottom) catalogs. Comparison with the dashed line, which has a slope of -0.52 , shows that the scaling with redshift is close to that expected. (Note that it is important to use volume-limited catalogs to make the measurement: the trend in any given volume-limited subsample is shallower than the trend one would infer by first combining together all the subsamples and then making the measurement. To see why this happens, suppose that the mean size at fixed luminosity scales as $R_{o} \propto L^{\alpha}$. Then the magnitude limit induces a correlation that should scale approximately as $r_{\mathrm{dev}} \propto R_{o} / z \propto L^{\alpha} / z \propto z^{2 \alpha} / z$. If $\alpha>1 / 2$, then $r_{\mathrm{dev}}$ increases with $z$, so $r_{10 \%} / r_{\mathrm{dev}}$ decreases with $z$ simply because of the magnitude limit.)

Some of the small differences in slope between the dashed and solid lines can be attributed to the effects of luminosity evolution. By fixing $L$, we select objects that are slightly smaller at higher $z$ (c.f. Fig. 12) hence have slightly smaller values of $r_{\mathrm{dev}}$ and so larger values of $r_{10 \%} / r_{\mathrm{dev}}$ than expected; as a result, they appear to decline less rapidly than const $-0.52 z$.

Luminosity evolution is expected to be stronger in the bluer bands, and the $g^{*}$-band relation does appear to be slightly shallower than the other bands. Thus, as was the case for $r_{\mathrm{dev}} / r_{\mathrm{PSF}}$, we see redshift-dependent trends in $r_{10 \%} /$ $r_{\mathrm{dev}}$ that we believe are physically reasonable. The sky subtraction procedure does not appear to have introduced any obvious artifacts into our estimates of $r_{\mathrm{dev}}$.
Finally, we turn to an inspection of how well the de Vaucouleurs' model actually fits the observed surface brightness profiles. Figure 7 shows angular averaged surface brightness profiles in the $g^{*}, r^{*}, i^{*}$, and $z^{*}$ bands (bottom to top in each of the top panels) for a random selection of galaxies in our sample, ordered by redshift and size. The text in each panel shows the plate number, MJD, and fiber identification number of the observation, the redshift $z$, the fitted apparent $r^{*}$ magnitude $m_{r^{*}}$, the fitted axis ratio $b / a$, and the effective physical radius $R_{o}$, computed from the fitted angular radius $r_{\mathrm{dev}}$, the fitted axis ratio, and the redshift, as described previously. The solid lines show de Vaucouleurs' profiles that, when convolved with the pipeline's model for the seeing, provide the best fit to the symbols (because the fits are actually not to these azimuthally averaged one-dimensional profiles, but to the two-dimensional profile, they serve mainly as guides to the eye.) The horizontal lines show the surface brightness at $1 \%$ of the sky in the different bands. Long, medium, and short tick marks on these lines show $r_{\mathrm{PSF}}, r_{\mathrm{dev}}$, and $r_{10 \%}$, the radius at which the fitted surface brightness equals $10 \%$ of the sky.

The bottom panels show the residuals (observed minus fit) in the different bands (crosses, stars, diamonds, and triangles show the $g^{*}, r^{*_{-}}, i^{*_{-}}$, and $z^{*}$-band results, respectively). The residuals are systematically low on small scales, but comparison with the large tick marks, which indicate the size of the seeing disk, show that this discrepancy is because we are comparing fits that have been corrected for seeing with the observed surface 


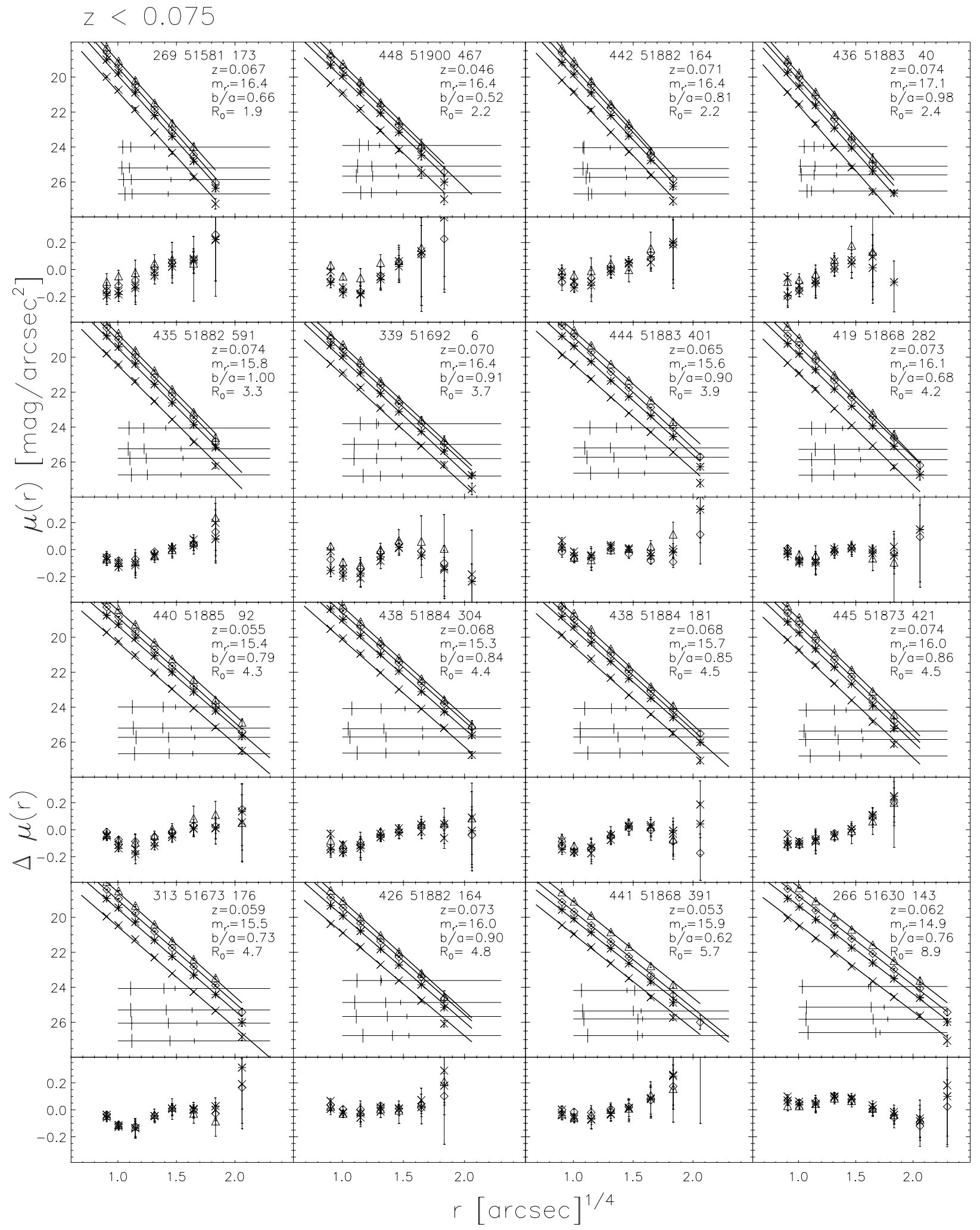

FIG. $7 a$

FIG. 7.-Examples of surface brightness profiles selected randomly from galaxies in our sample that are in the redshift range $(a) z<0.075$, $(b)$ $0.075<z<0.1,(c) 0.1<z<0.12,(d) 0.12<z<0.14$, (e) $0.14<z<0.18$, and $(f) z>0.18$. Top panels: Text in each panel shows plate number, MJD, and fiber identification of the object, the redshift $z$, the apparent $r^{*}$ magnitude $m_{r^{*}}$, the fitted axis ratio $b / a$, and the physical half-light radius $R_{o}$. Panels are ordered by physical radius $R_{o}$, which increases from top to bottom and from left to right. Symbols show the surface brightness profiles in the $g^{*}, r^{*}, i^{*}$, and $z^{*}$ bands (bottom to top in each of the top panels), and solid lines show the de Vaucouleurs' fits, which when convolved with the seeing, provide the best fit to the symbols. Horizontal lines show $1 \%$ of the surface brightness of the sky in the different bands, and large, medium, and small tick marks show $r_{\mathrm{PSF}}, r_{\mathrm{dev}}$, and $r_{10 \%}$, the radius at which the fitted surface brightness equals $10 \%$ of the sky. Bottom panels: Residuals (observed minus fit) in the different bands (crosses, stars, diamonds, and triangles show the $g^{*}-, r^{*}-, i^{*}$, and $z^{*}$-band results, respectively). 


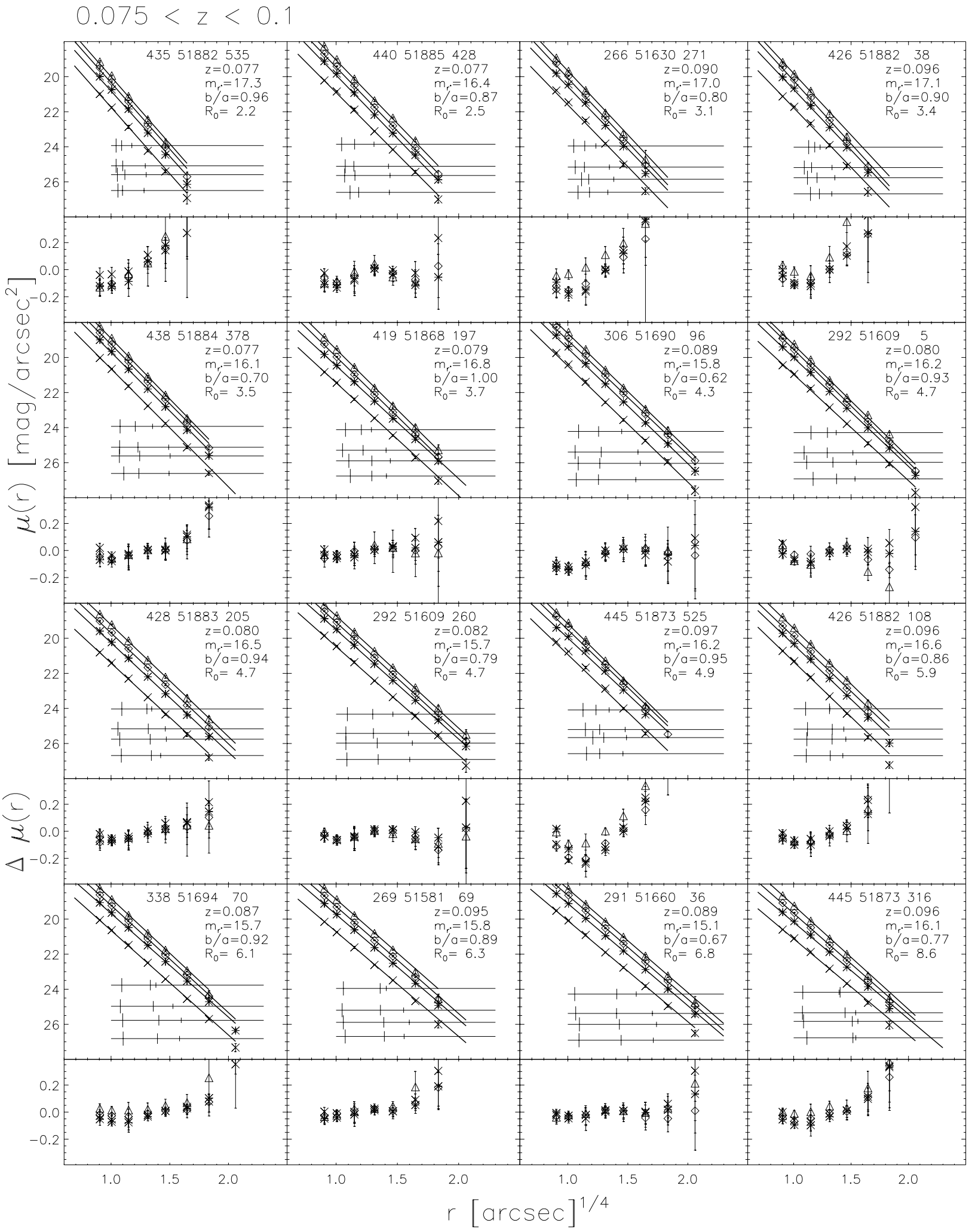

FIG. $7 b$

brightnesses that are not seeing-corrected. On larger scales (typically larger than twice the half-light radii), the residuals are also large, indicating that there may be problems with the sky subtraction. In linear flux units, the residuals tend to a constant value that is typically smaller than $0.3 \%$ of the sky level (recall that the hori- zontal lines in the panels show the surface brightness at $1 \%$ of sky). Although this is not an entirely fair comparison, since the fits are not performed on these azimuthally averaged one-dimensional profiles, but on the twodimensional profile, this test suggests that the fit is reliable, but that the zero-point level of the sky may be 


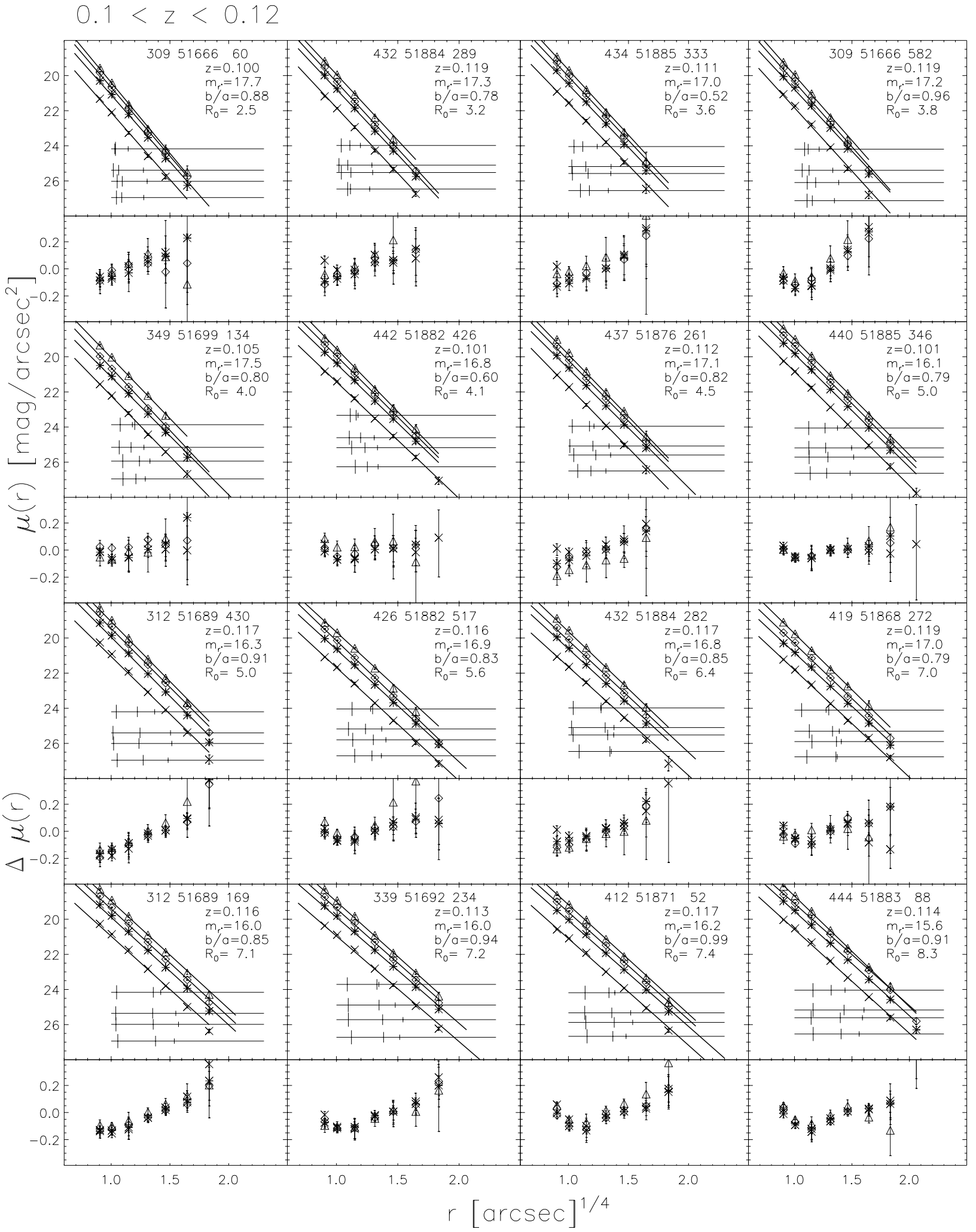

FIG. $7 c$

systematically biased by $\sim 0.3 \%$. Note that the residuals are larger in the $g^{*}$ and $z^{*}$ bands; but this is consistent with the larger errors we quote on the photometry in these bands.

Figure 8 shows the $r^{*}$ images of the same objects whose profiles are shown in Figure 7. The images are each
$11.88 \times 11.88 \operatorname{arcsec}^{2}$ (i.e., 30 pixels on a side). One might expect some of the worst fits are because of the presence of a disk component that our de Vaucouleurs' model does not account for. However, comparison of the images and the fits shows no obvious correlation between goodness of fit and, e.g., $b / a$. 


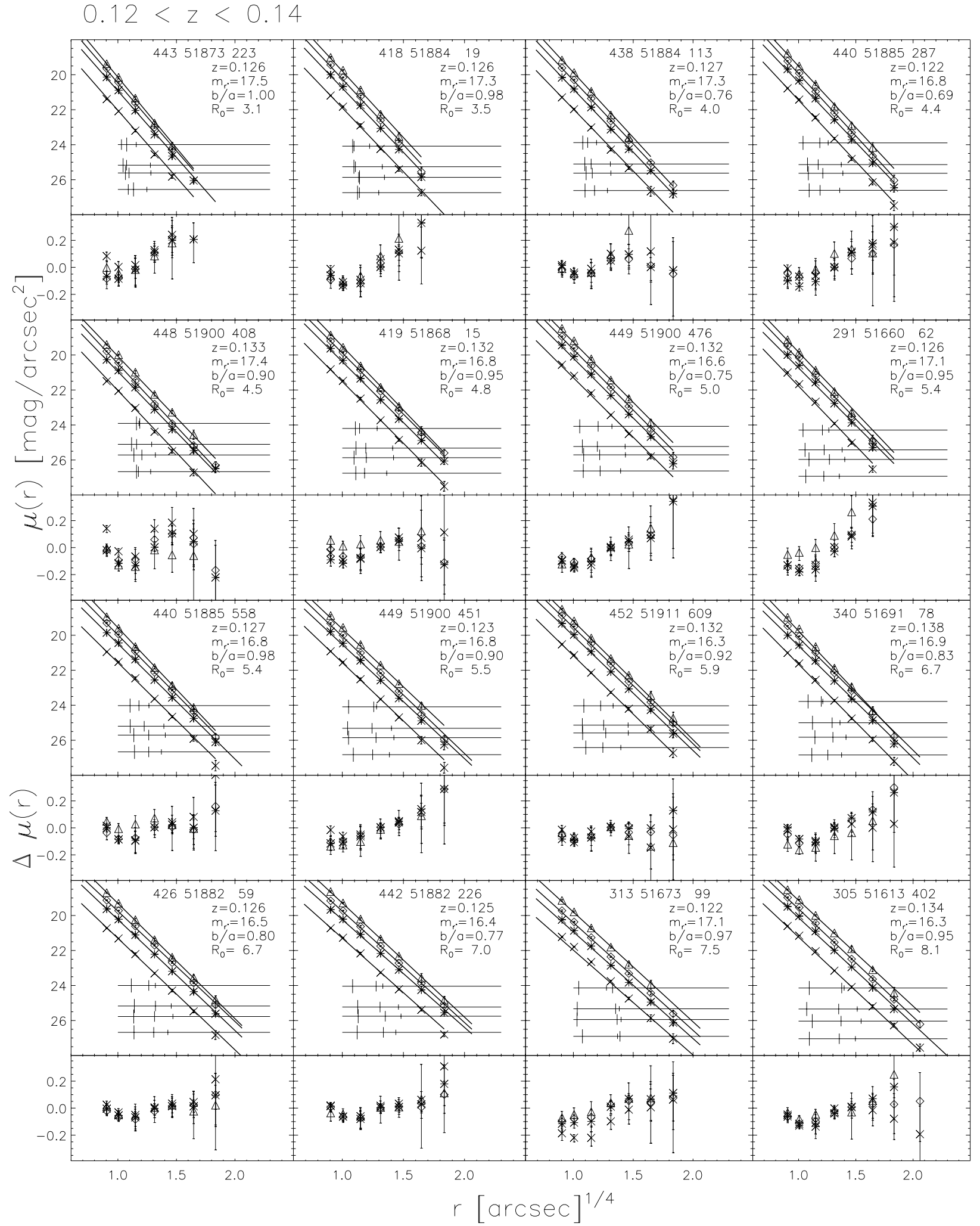

FIG. $7 d$

In summary, we have performed a number of tests of the SDSS photometric pipeline reductions and have found little evidence that they are significantly biased. Therefore, in what follows, the apparent magnitudes we use are those output by the SDSS photometric pipeline. To convert to luminosities, we $K$-correct (and use the inverse square law). The angular sizes are output from the SDSS photometric pipeline. We correct these to a fixed rest-frame size, and we correct for $a / b$. Both these corrections are standard. Thereafter, surface brightness is defined from the rest-frame luminosity and size with no additional corrections. The uncertainties on the 


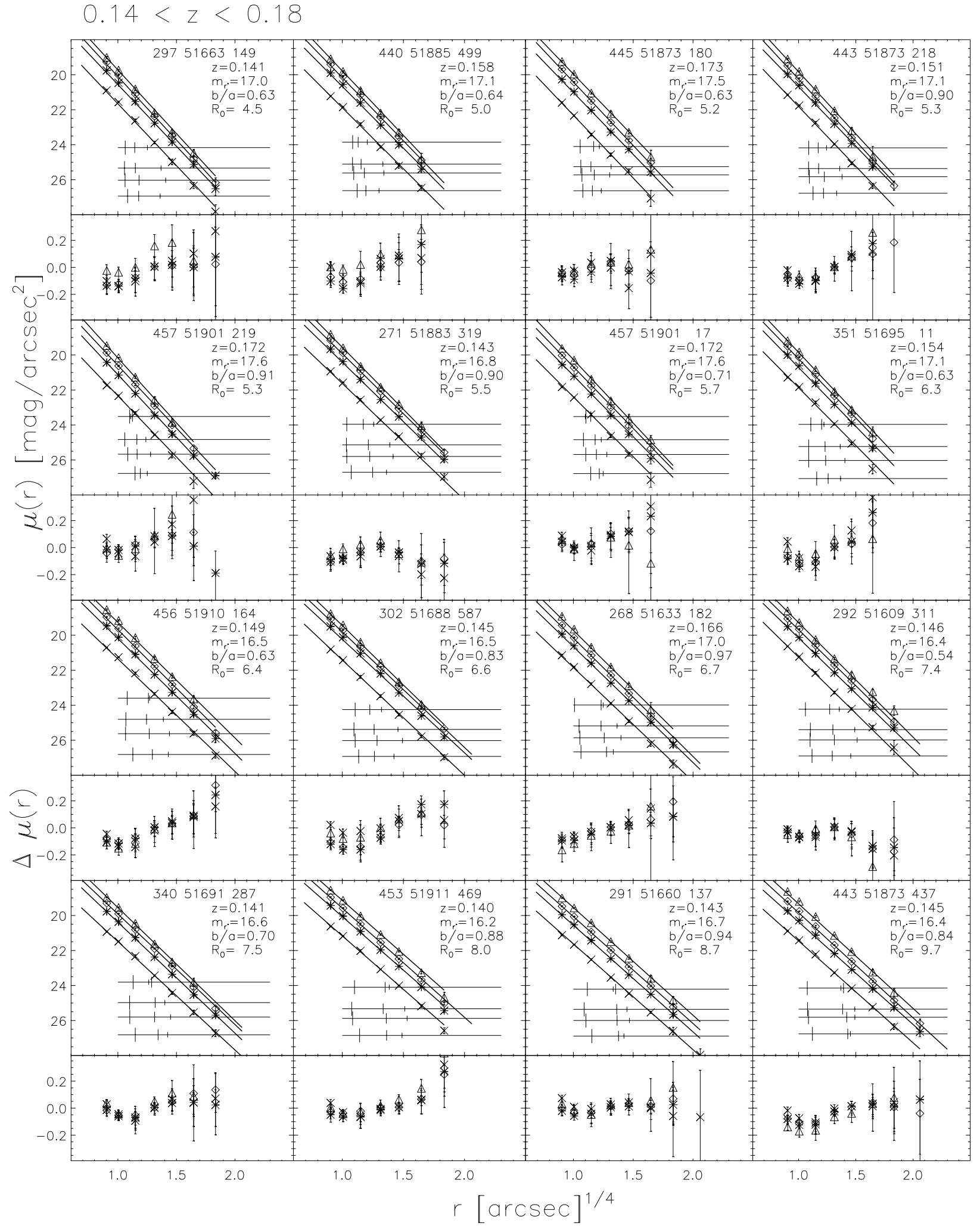

FIG. $7 e$

photometric parameters we use in this paper and the magnitude limits in each band are summarized in Table 1. There is some subtlety in obtaining them from the format available in the SDSS database; this is discussed in Appendix D.

\subsection{Spectroscopic Data of the Sample}

\subsubsection{Estimating the Velocity Dispersions}

The SDSS pipeline does not provide an estimate of the line-of-sight velocity dispersion, $\sigma$, within a galaxy, so we 


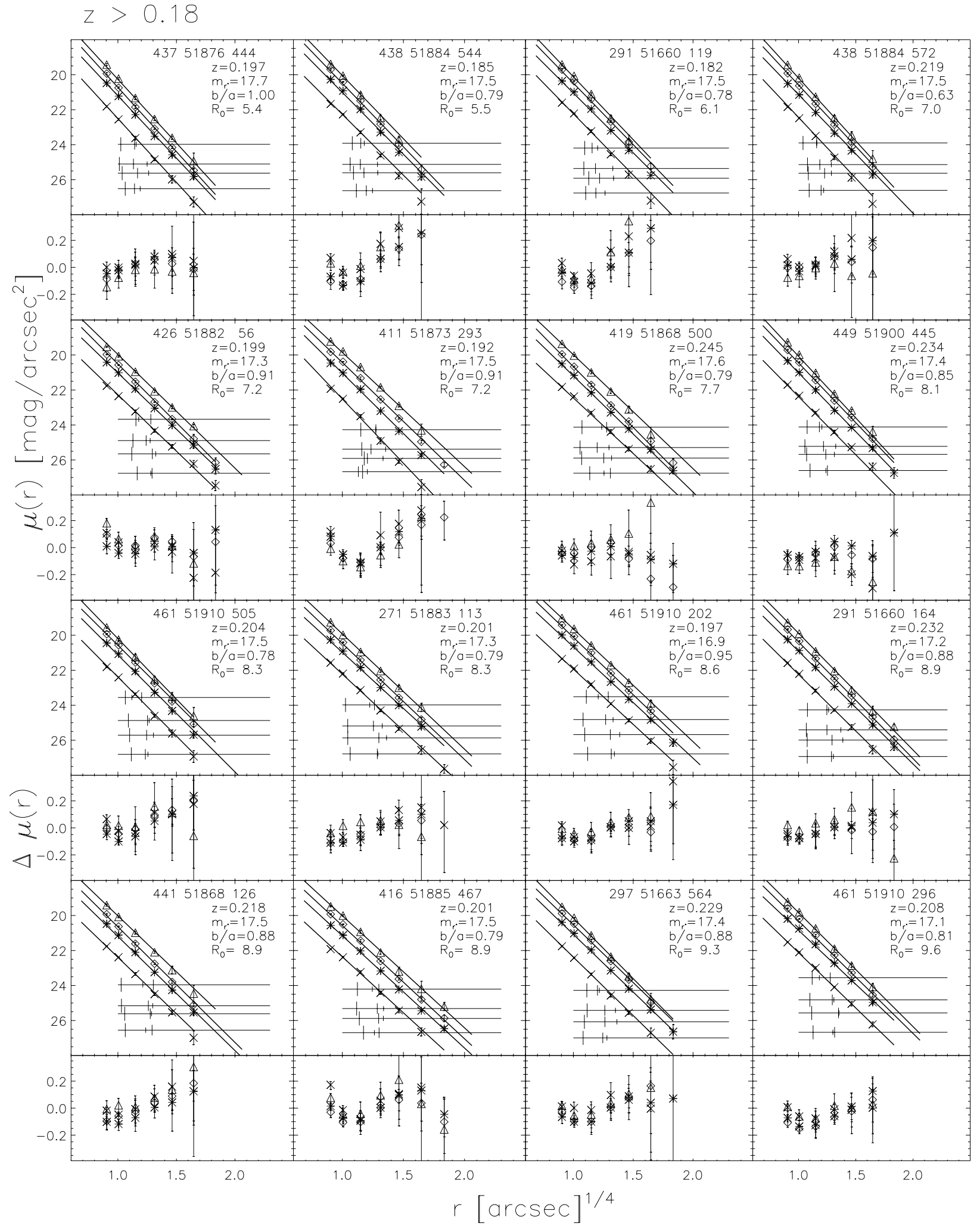

FIG. $7 f$

must compute it separately for the early-type galaxy sample. The observed velocity dispersion $\sigma$ is the result of the superposition of many individual stellar spectra, each of which has been Doppler-shifted because of the star's motion within the galaxy. Therefore, it can be determined by analyzing the integrated spectrum of the whole galaxy.
A number of objective and accurate methods for making velocity dispersion measurements have been developed (Sargent et al. 1977; Tonry \& Davis 1979; Franx, Illingworth, \& Heckman 1989; Bender 1990; Rix \& White 1992). Each of these methods has its own strengths, weaknesses, and biases. Appendix B describes how we 


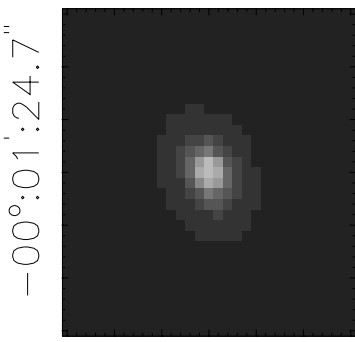

$10^{\mathrm{h}}: 04^{\mathrm{m}}: 57.1^{\mathrm{s}}$

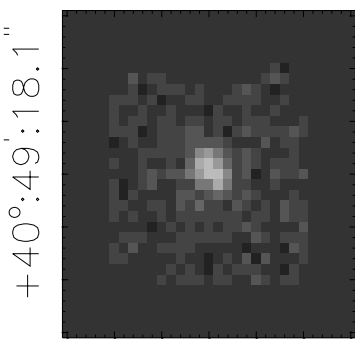

$07^{\mathrm{h}}: 58^{\mathrm{m}}: 13.4^{\mathrm{s}}$

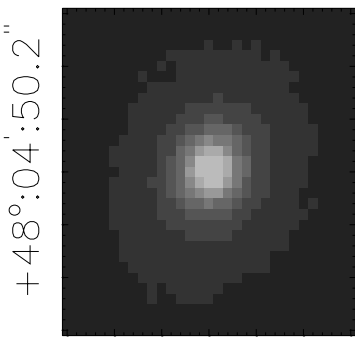

$08^{\mathrm{h}}: 15^{\mathrm{m}}: 10.1^{\mathrm{s}}$

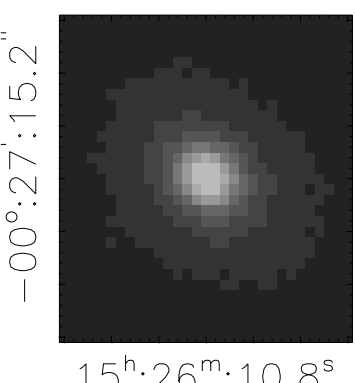

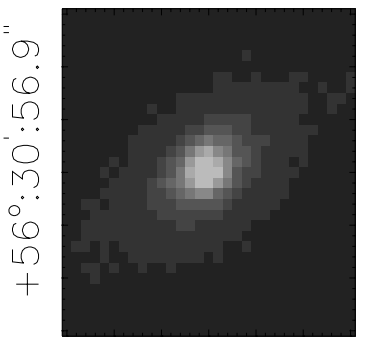

$08^{\mathrm{h}}: 49^{\mathrm{m}}: 14.9^{\mathrm{s}}$
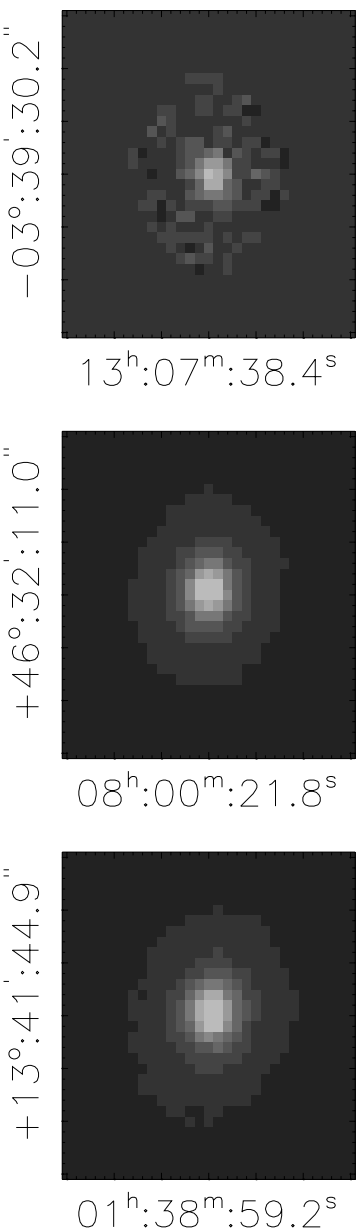
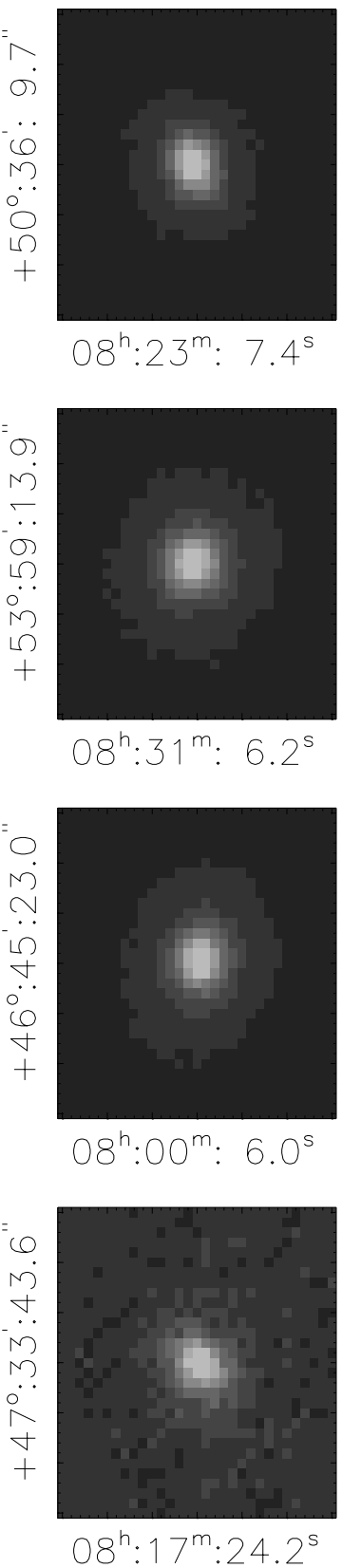

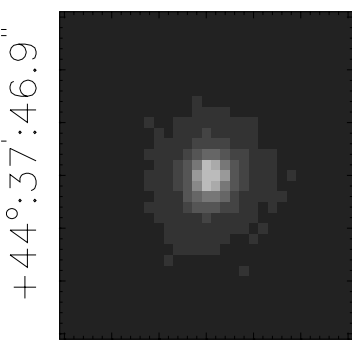

$08^{\mathrm{h}}: 03^{\mathrm{m}}: 30.7^{\mathrm{s}}$
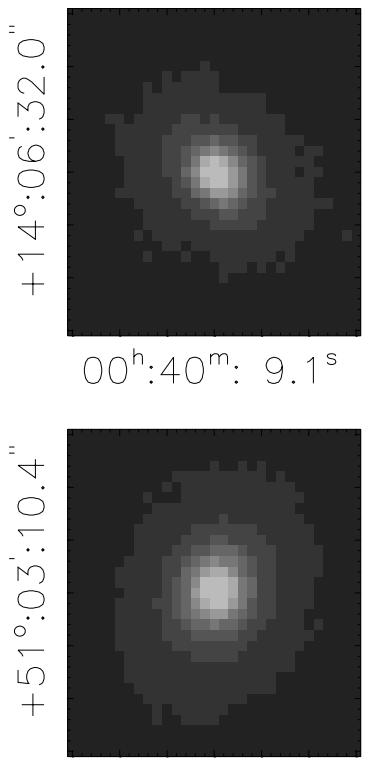

$08^{\mathrm{h}}: 34^{\mathrm{m}}: 10.8^{\mathrm{s}}$

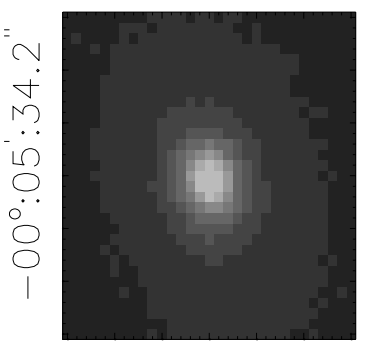

$09^{\mathrm{h}}: 45^{\mathrm{m}}: 52.6^{\mathrm{s}}$

FIG. $8 a$

FIG. 8. - The $r^{*}$-band images for the $(a) z<0.075,(b) 0.075<z<0.1,(c) 0.1<z<0.12,(d) 0.12<z<0.14,(e) 0.14<z<0.18$, and $(f) z>0.18$ objects shown in Fig. 7. The labels on the $x$ - and $y$-axes show the coordinates (R.A. and decl. in J2000.0) of the galaxy centers.

combined these different techniques to estimate $\sigma$ for the galaxies in our sample.

The velocity dispersion estimates we use in what follows are obtained by fitting the rest-frame wavelength range 4000-7000 $\AA$ and then averaging the estimates provided by the Fourier-fitting and direct-fitting methods to define what we call $\sigma_{\text {est. }}$ (We do not use the cross-correlation estimate because of its behavior at low $\mathrm{S} / \mathrm{N}$ as discussed in Appendix B. Note that the $\mathrm{S} / \mathrm{N}$ of the SDSS spectra depend on wavelength.) The error on $\sigma_{\text {est }}$ is determined by adding in quadrature the errors on the two estimates (i.e., the Fourier-fitting and direct-fitting) that we averaged. The resulting error is between $\delta \log \sigma \sim 0.02$ dex and 0.06 dex, depending on the $\mathrm{S} / \mathrm{N}$ of the spectra, with a median value of $\sim 0.03$ dex. A few galaxies in our sample have been observed more than once.
The scatter between different measurements is $\sim 0.04$ dex, consistent with the amplitude of the errors on the measurements (see Fig. 19). Based on the typical S/N of the SDSS spectra and the instrumental resolution, we chose $70 \mathrm{~km} \mathrm{~s}^{-1}$ as a lower limit on the velocity dispersions we use in this paper.

Following Jørgensen, Franx, \& Kjærgaard (1995) and Wegner et al. (1999), we correct $\sigma_{\text {est }}$ to a standard relative circular aperture defined to be one-eighth of the effective radius:

$$
\frac{\sigma_{\text {cor }}}{\sigma_{\text {est }}}=\left(\frac{r_{\text {fiber }}}{r_{o} / 8}\right)^{0.04},
$$

where $r_{\text {fiber }}=1 " .5$ and $r_{o}$ is the effective radius of the galaxy 

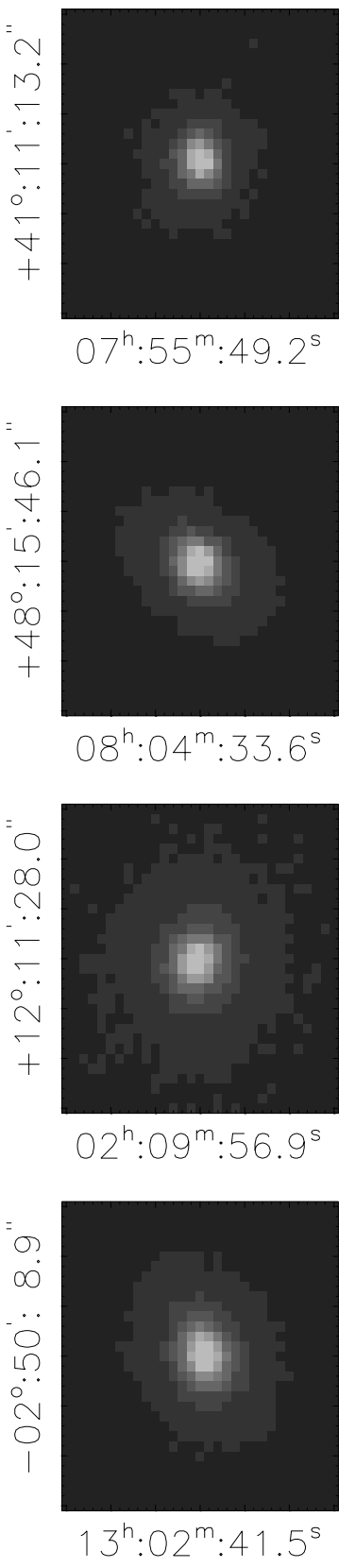
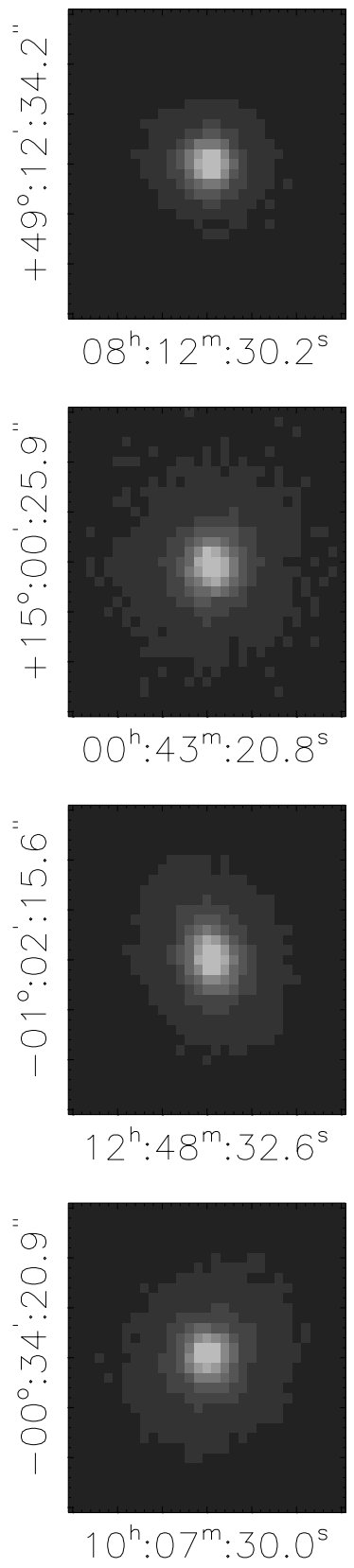
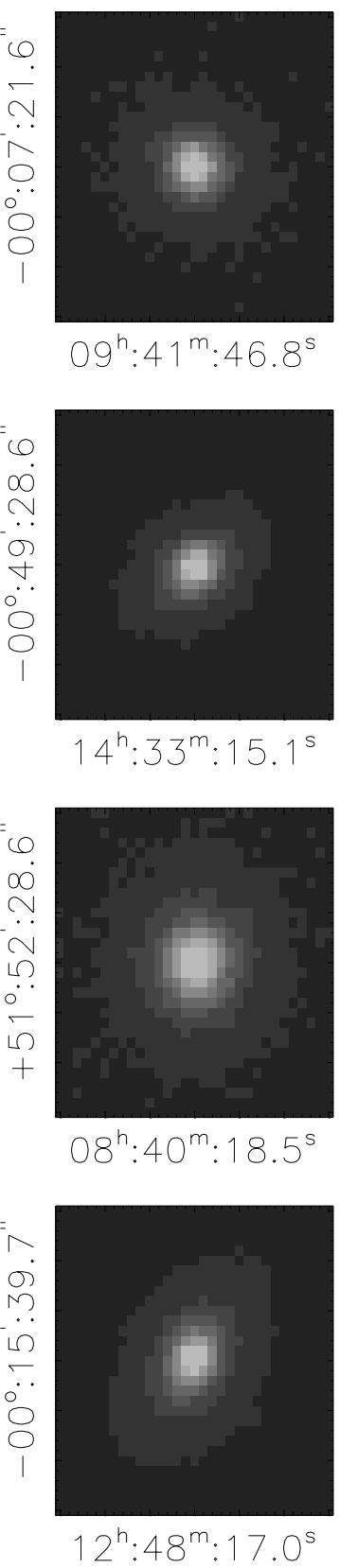

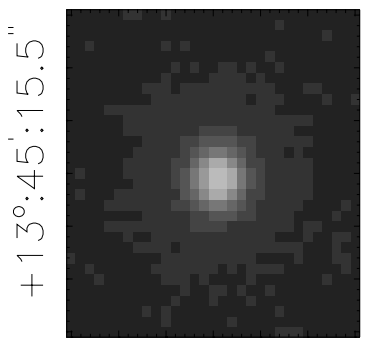

$01^{\mathrm{h}}: 44^{\mathrm{m}}: 14.2^{\mathrm{s}}$
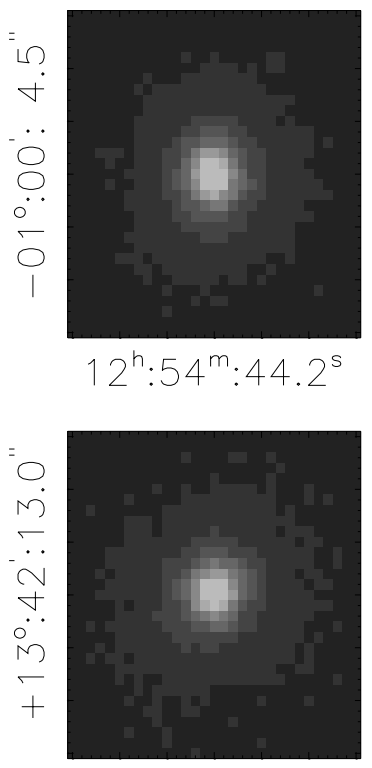

$01^{\mathrm{h}}: 41^{\mathrm{m}}: 15 \cdot 4^{\mathrm{s}}$

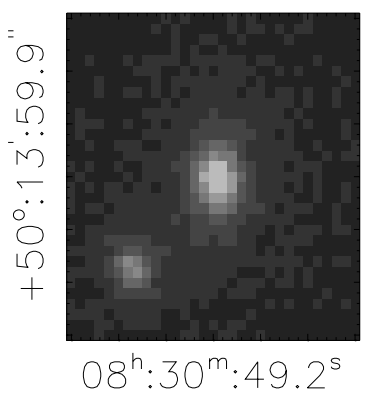

FIG. $8 b$

measured in arcseconds. In principle, we should also account for the effects of seeing on $\sigma_{\text {est }}$, just as we do for $r_{o}$. However, because the aperture correction depends so weakly on $r_{o}$ (as the 0.04 power), this is not likely to be a significant effect. In any case, most galaxies in our sample have $r_{o} \geq 1$ '!5 (see Fig. 3).

Note that this correction assumes that the velocity dispersion profiles of early-type galaxies having different $r_{o}$ are similar. At the present time, we do not have measurements of the profiles of any of the galaxies in our sample, so we cannot test this assumption. In Paper II of this series, we will argue that the galaxies in our sample evolve very little; this means that if we select galaxies of the same luminosity and effective radius, then a plot of velocity dispersion versus redshift of these objects should allow us to determine if the aperture correction above is accurate. The results of this exercise are presented in Appendix C.

\subsubsection{Examples of Early-Type Galaxy Spectra}

To illustrate the typical quality of the spectra in our sample, Figure 9 shows spectra of the galaxies that lie along the diagonals in Figure 7 (panels from top to bottom here are for objects that are top left to bottom right in Fig. 7). All the spectra in our sample have PCA classification numbers $a<-0.1$, typical of early-type galaxy spectra (Connolly \& Szalay 1999), although a few of the spectra do show weak emission lines.

The typical $\mathrm{S} / \mathrm{N}$ of the spectra in our sample is 16 , although $\mathrm{S} / \mathrm{N}$ does vary with wavelength. Therefore, individual spectra cannot be used to make reliable estimates of, 


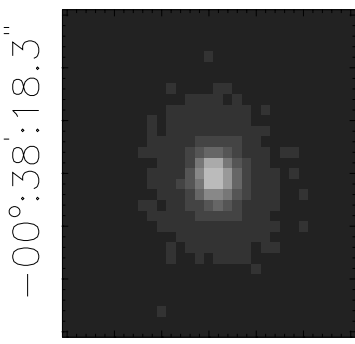

$14^{\mathrm{h}}: 57^{\mathrm{m}}: 19 \cdot 4^{\mathrm{s}}$
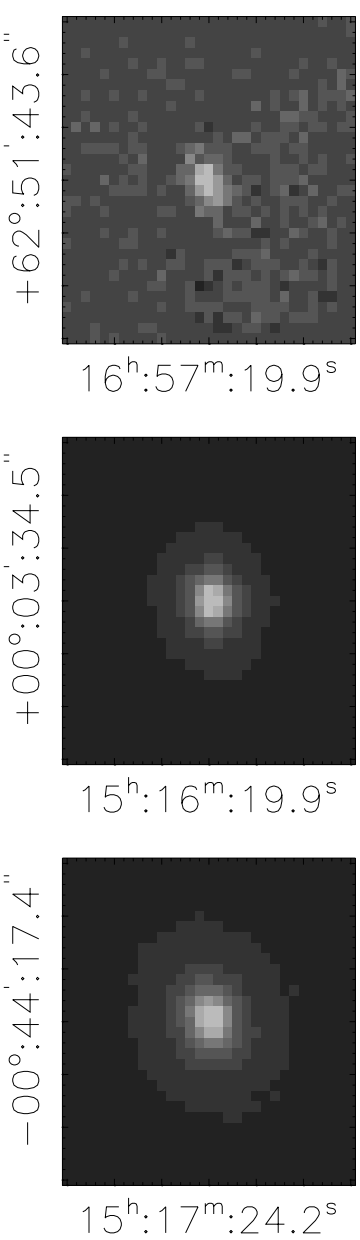

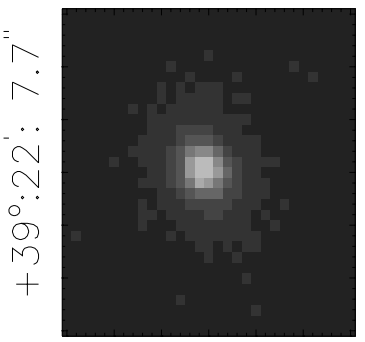

$07^{\mathrm{h}}: 35^{\mathrm{m}}: 22.6^{\mathrm{s}}$
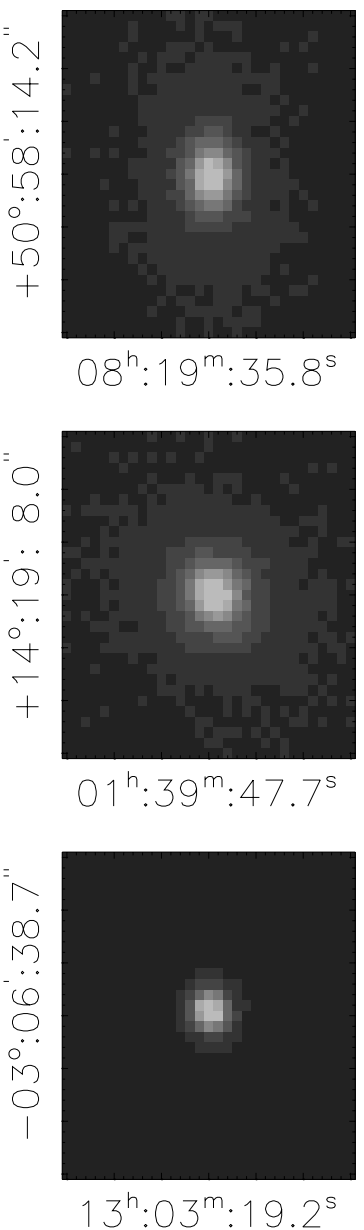
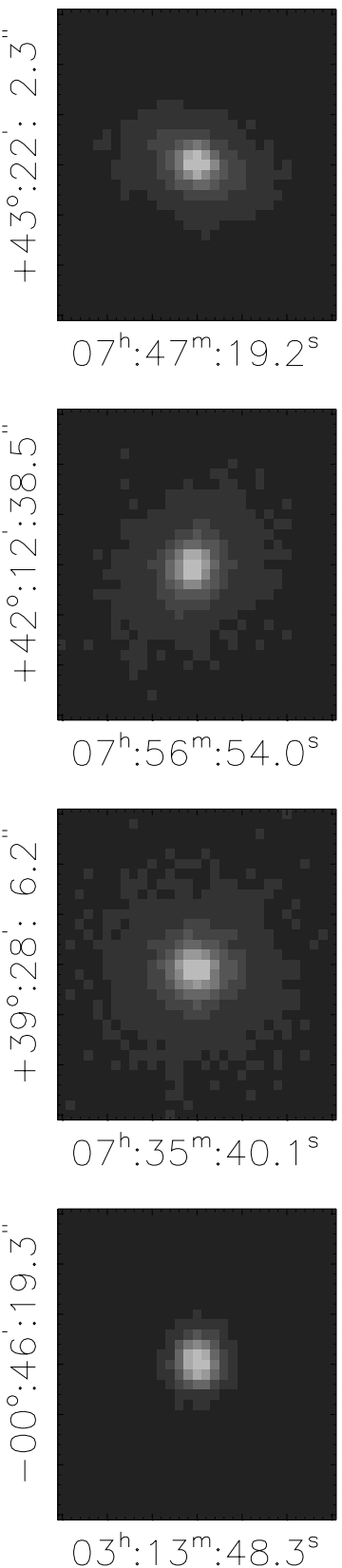

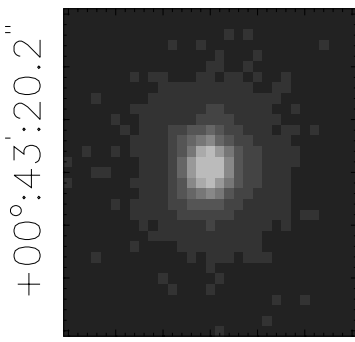

$14^{\mathrm{h}}: 58^{\mathrm{m}}: 21.8^{\mathrm{s}}$

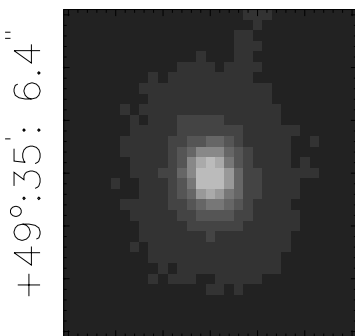

$08^{\mathrm{h}}: 09^{\mathrm{m}}: 5.8^{\mathrm{s}}$

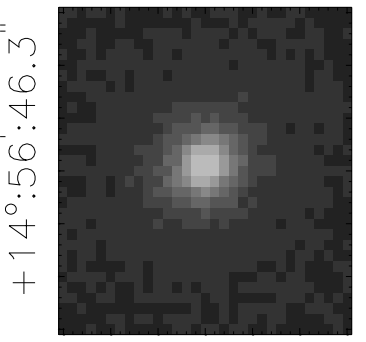

$00^{\mathrm{h}}: 41^{\mathrm{m}}: 1.8^{\mathrm{s}}$

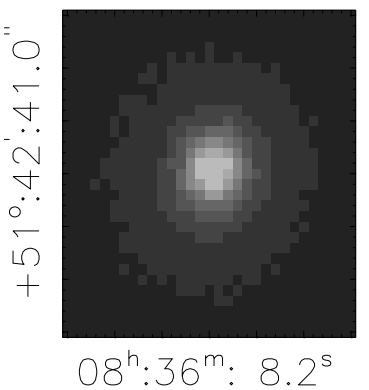

FIG. $8 c$

e.g., the Lick indices. However, by co-adding spectra of similar objects (i.e., galaxies with similar luminosities, sizes, velocity dispersions, and redshifts), it is possible to construct composite spectra that have considerably better $\mathrm{S} / \mathrm{N}$. This is the subject of Paper IV.

\subsection{An Estimate of the Local Density}

In subsequent papers, we will be interested in how the properties of the galaxies in our sample depend on their local environment. To do so, we must come up with a working definition of environment. The set of galaxies in the SDSS photometric database is much larger than those for which the survey actually measures redshifts. Some of these galaxies may well lie close to galaxies in our sample, in which case they will contribute to the local density. We would like to find some way of accounting for such objects when we estimate the local density.

For a subset of the galaxies in our sample, the colors expected of a passively evolving early-type were used to select a region in $g^{*}-r^{*}$ versus $r^{*}-i^{*}$ color space at the redshift of the galaxy of interest. All galaxies within $0.1 \mathrm{mag}$ in color of this point were included if they were (1) within $1 h^{-1}$ Mpc of the main galaxy and (2) brighter than -20.25 in $M_{i *}$. (The box in color space is sufficiently large that the difference between this technique, and using the observed colors themselves to define the selection box is not important.) These two cuts are made assuming every galaxy in the colorcolor range is at the redshift of the galaxy of interest. The end result of this is that each galaxy in the subsample is assigned a number of neighbors. Note that, because of the selection on color, our estimate of the local density is 

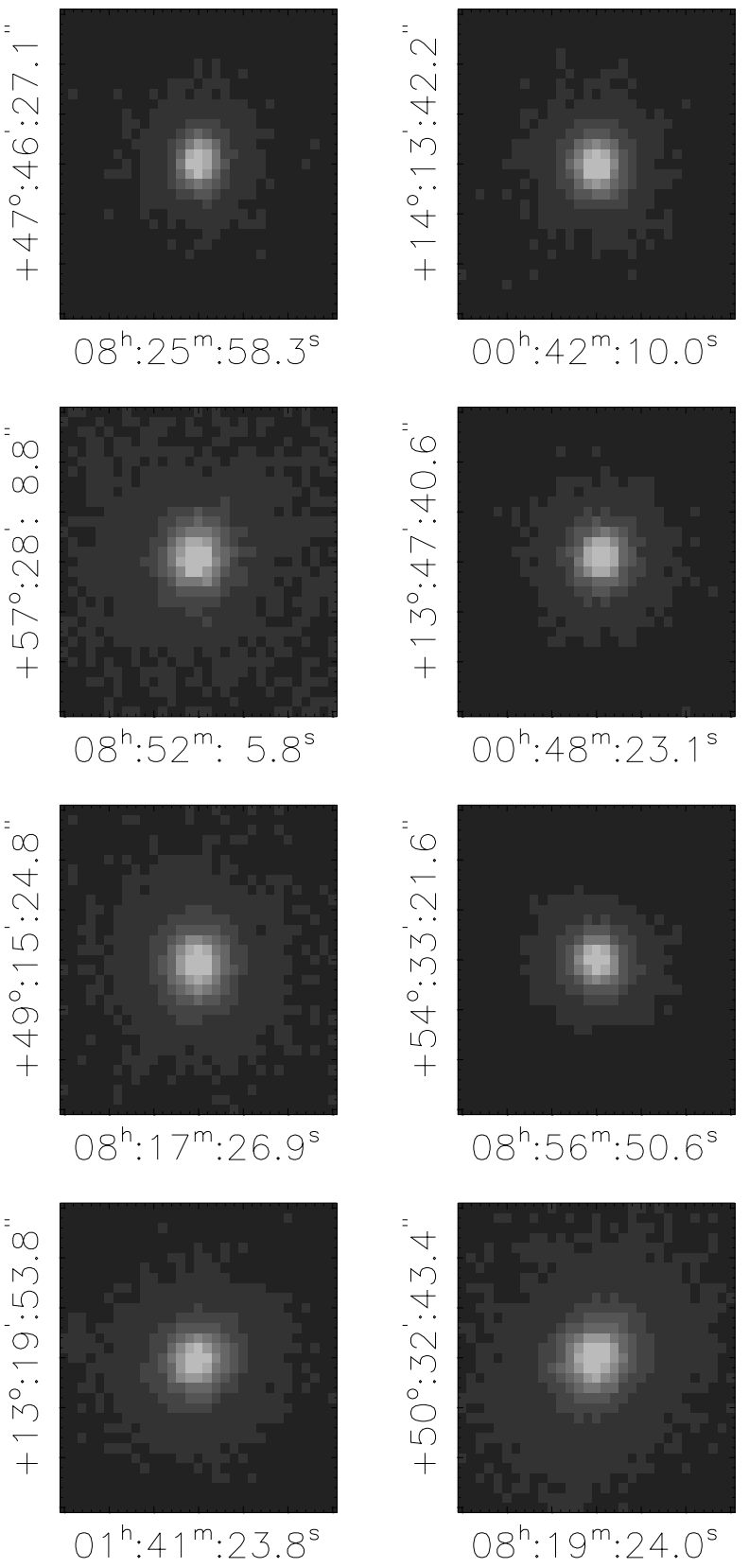
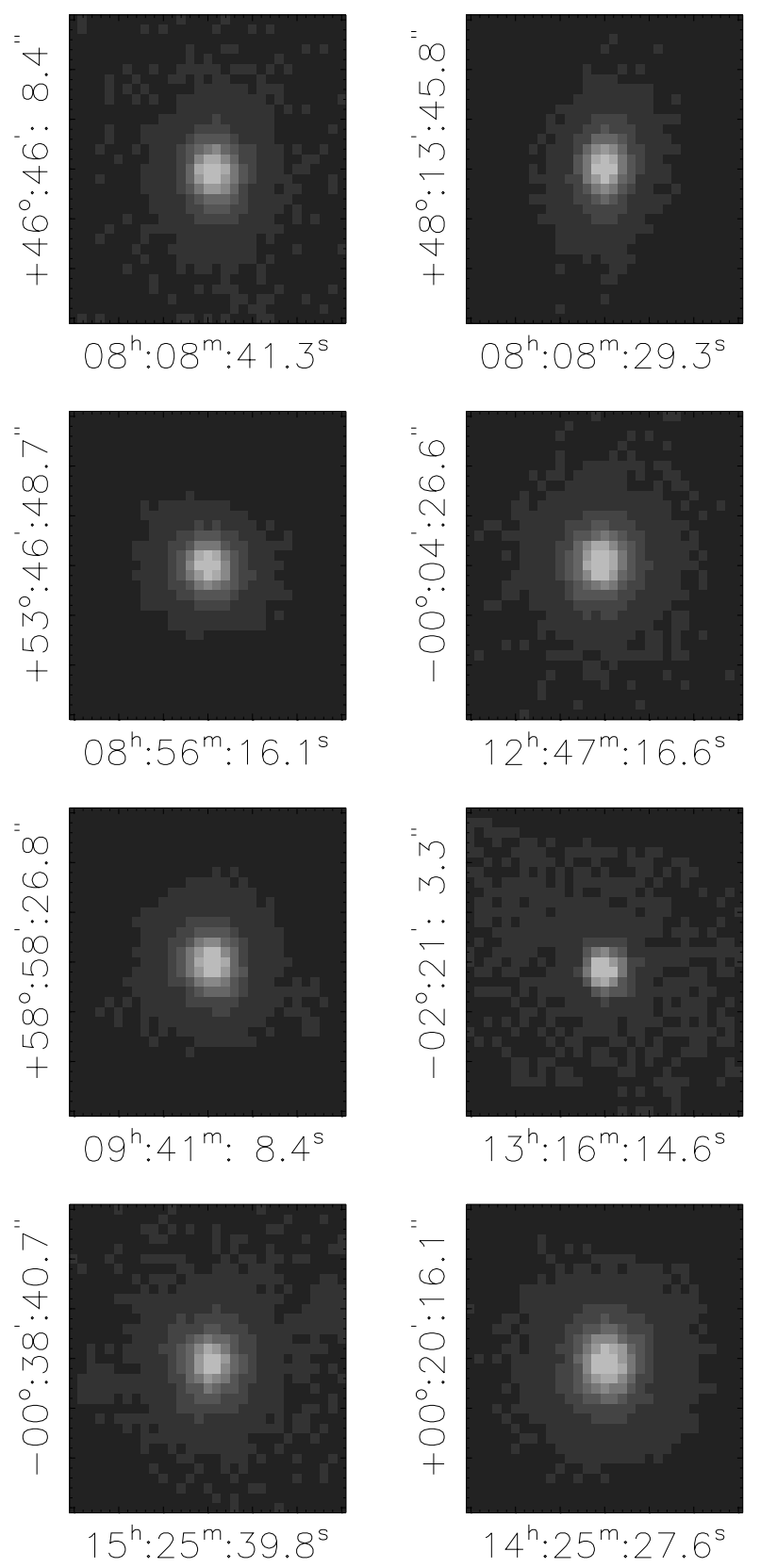

FIG. $8 d$

actually an estimate of the number of neighbors that have the same colors as early-type galaxies. In the papers that follow, we will often present results for different redshift bins. When we do, it is important to bear in mind that this procedure for assigning neighbors is least secure in the lowest redshift bin (typically $z \leq 0.08$ ).

Figure 10 shows how the luminosities, surface brightnesses, sizes, velocity dispersions, and (a combination of the) axis ratios depend on environment. The different symbols for each bin in density show averages over galaxies in different redshift bins: circles, diamonds, triangles, and squares are for galaxies with redshifts in the range $0.075<$ $z \leq 0.1,0.1<z \leq 0.12,0.12<z \leq 0.14$, and $0.14<z \leq$ 0.18 . Error bars show the error in the mean value for each bin. Symbols for the higher redshift bins have been offset slightly to the right.
For any given set of symbols, the bottom right panel shows that the mean redshift in each bin in density is not very different from the mean redshift averaged over all bins. This suggests that our procedure for estimating the local densities is not biased. The other panels show corresponding plots for the other observed parameters. When the number of near neighbors is small, the luminosities, sizes, and velocity dispersions all increase slightly as the local density increases, whereas the surface brightnesses decrease slightly. All these trends are very weak. The bottom right panel shows $(a / b-1)^{1 / 2}-\left\langle(a / b-1)^{1 / 2}\right\rangle$, where $b / a$ is the axis ratio. [Some authors (e.g., de Zeeuw \& Franx 1991) suggest that this is a good approximation of the ratio of rotational to random motions within the galaxy, $(v / \sigma)_{\text {iso }}$, for oblate, rotationally flattened galaxies. Bright elliptical galaxies have been shown to have a mean $(v / \sigma) /(v / \sigma)_{\text {iso }} \sim 0.4$.] 

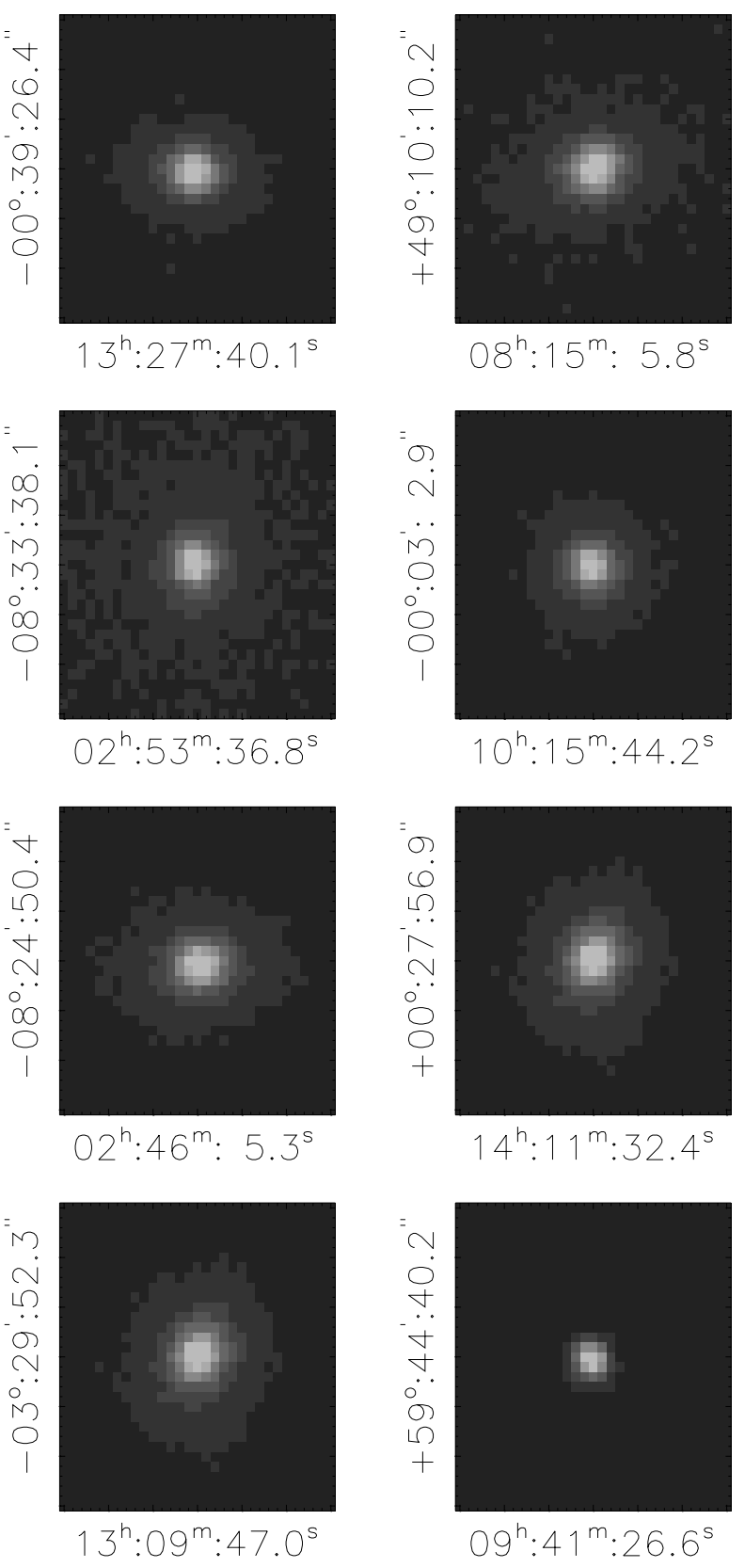
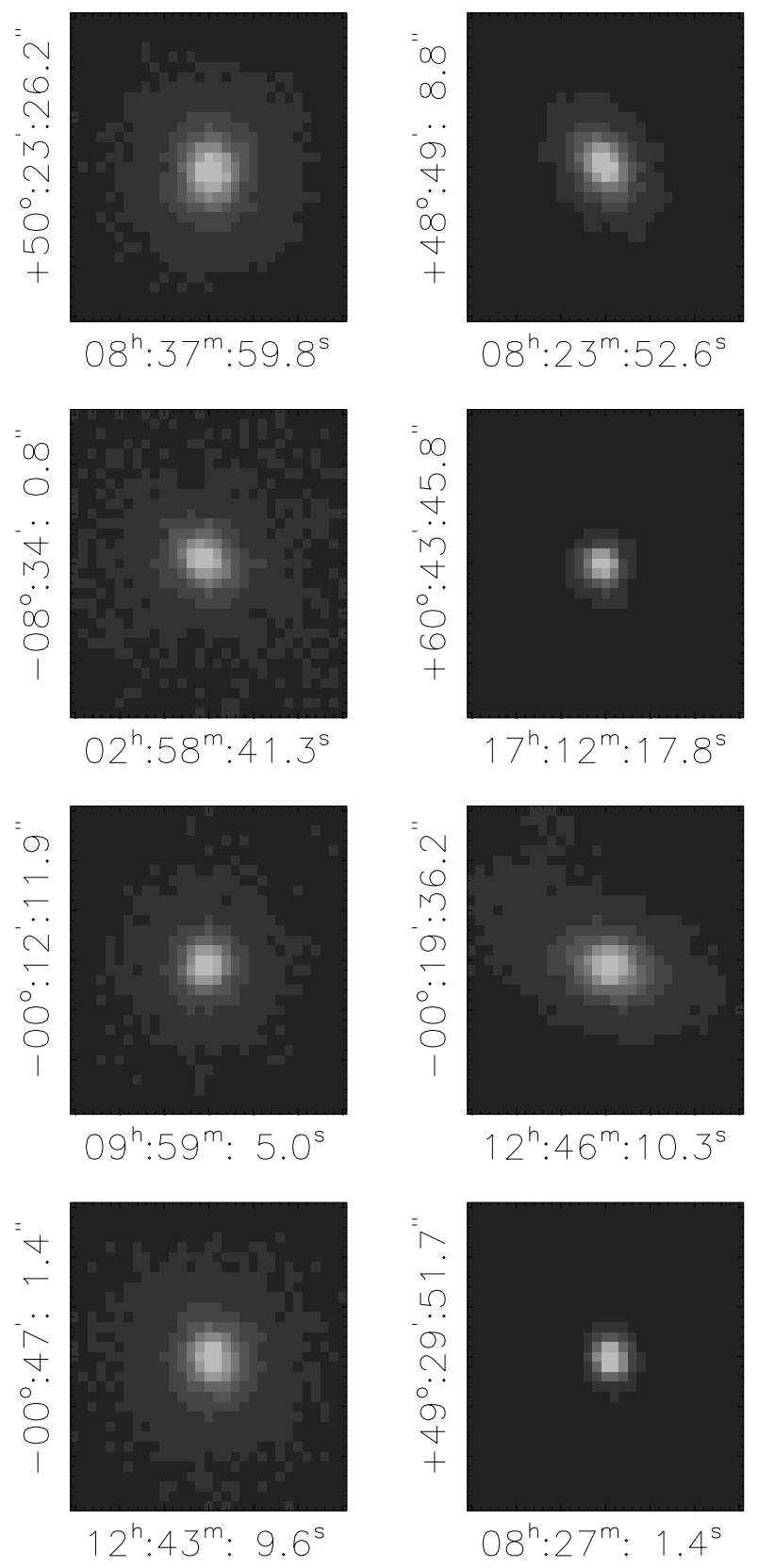

FIG. $8 e$

There are no obvious trends with environment. It is difficult to say with certainty that the trends with environment in the top four panels of Figure 10 are significant. A more efficient way of seeing whether the properties of galaxies depend on environment is to show the residuals from the fundamental plane. This is done in Paper III.

Figure 11 shows a redshift-space pie-diagram distribution of our sample. Most of the sample is at low declination $\left(|\delta| \leq 2^{\circ}\right)$; in addition, there are three wedges from three different disconnected regions on the sky.

\section{THE EARLY-TYPE GALAXY CATALOG}

The observed parameters of each galaxy in our earlytype galaxy sample are given in Table 2. The different columns show the R.A., decl., redshift $z$ and its error estimate, the $\mathrm{S} / \mathrm{N}$ of the spectrum, the apparent half-light radius $r_{\mathrm{dev}}$ and error estimate, the fitted apparent magnitude $m_{\mathrm{dev}}$ and error estimate, the model magnitude $m_{m}$ and error estimate, and the axis ratio $b / a$ and error estimate. The photometric parameters in this table are those measured in the $r^{*}$ band. Because we will be interested in the distribution of colors for a galaxy sample defined in, e.g., the $r^{*}$ band, the table presents the parameters of the same galaxies in all the bands. To select the magnitudelimited samples we use in subsequent papers, refer to Table 1 . The errors $\delta m_{\text {dev-photo }}$ and $\delta m_{\text {mod-photo }}$ are the error estimates output by the SDSS photometric pipeline. As discussed in Appendix D, they are not appropriate estimates of the errors in $m_{\mathrm{dev}}$ and $m_{\text {model }}$. Our procedure for making more appropriate error estimates is described in Appendix D.

Table 3 shows rest-frame quantities we computed from the quantities in Table 2. The different columns show the 


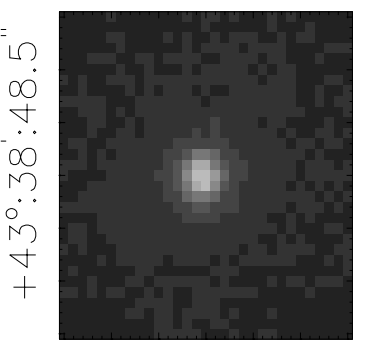

$08^{\mathrm{h}}: 02^{\mathrm{m}}: 37 \cdot 0^{\mathrm{s}}$

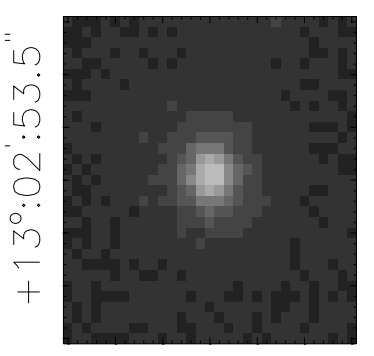

$01^{\mathrm{h}}: 41^{\mathrm{m}}: 55.3^{\mathrm{s}}$

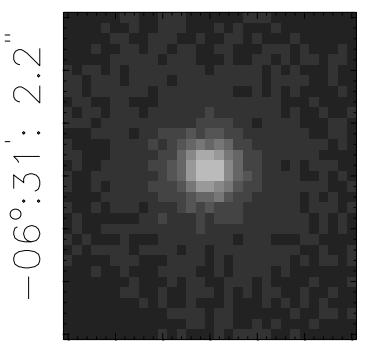

$03^{\mathrm{h}}: 33^{\mathrm{m}}: 5.6^{\mathrm{s}}$

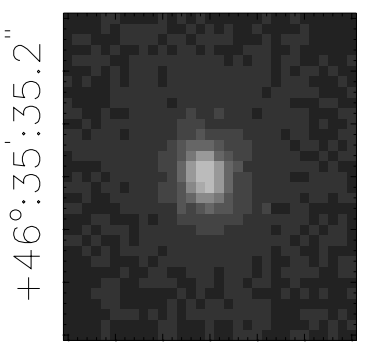

$08^{\mathrm{h}}: 20^{\mathrm{m}}: 15.1^{\mathrm{s}}$

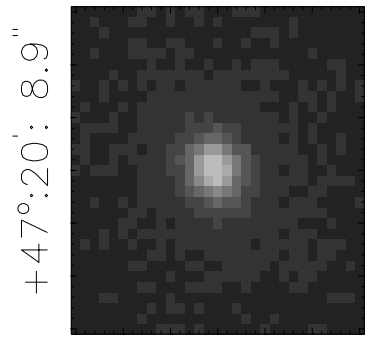

$08^{\mathrm{h}}: 08^{\mathrm{m}}: 1.9^{\mathrm{s}}$

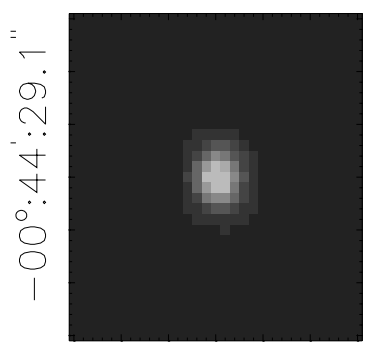

$02^{h}: 58^{m}: 20 \cdot 3^{s}$

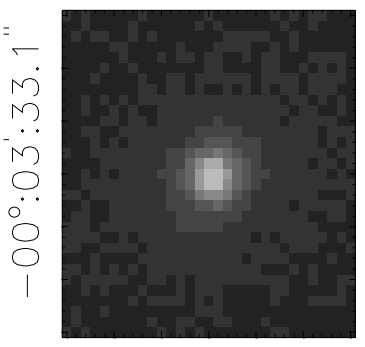

$10^{h}: 19^{m}: 53.5^{s}$

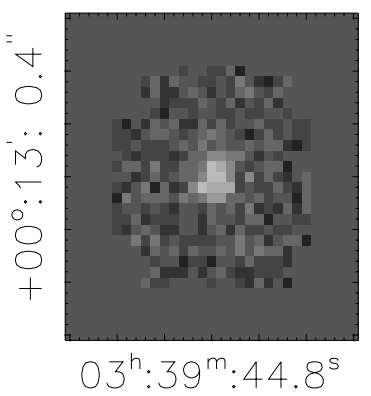

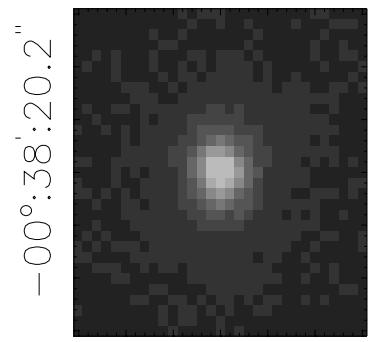

$12^{\mathrm{h}}: 46^{\mathrm{m}}: 55.2^{\mathrm{s}}$

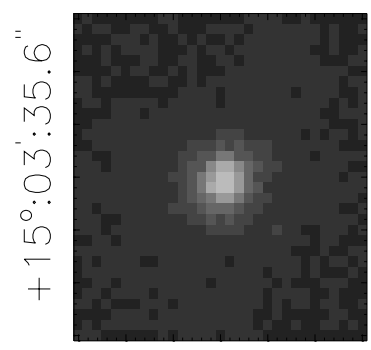

$00^{\mathrm{h}}: 44^{\mathrm{m}}: 17.2^{\mathrm{s}}$

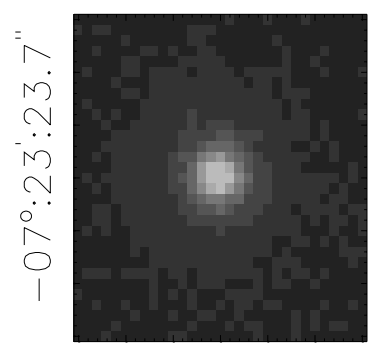

$03^{\mathrm{h}}: 31^{\mathrm{m}}: 18.1^{\mathrm{s}}$

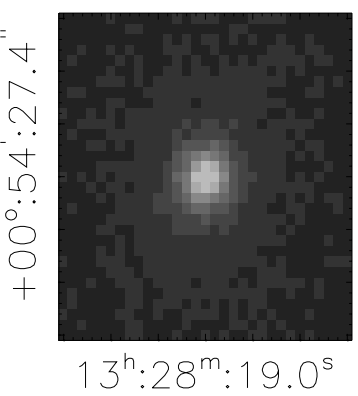

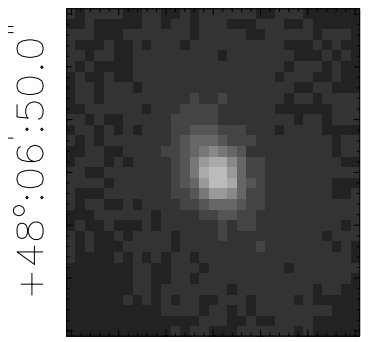

$08^{\mathrm{h}}: 08^{\mathrm{m}}: 24.0^{\mathrm{s}}$

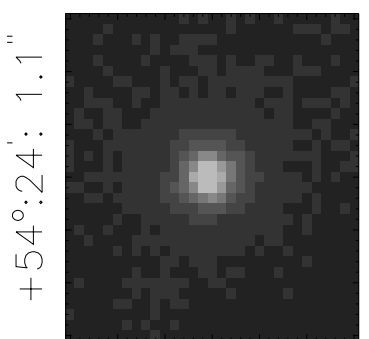

$08^{\mathrm{h}}: 57^{\mathrm{m}}: 47.3^{\mathrm{s}}$

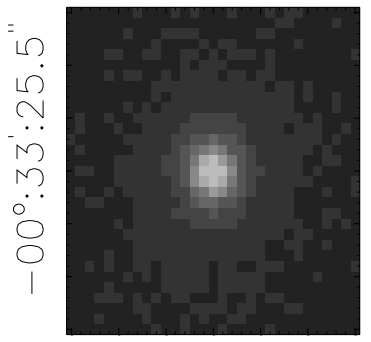

$12^{h}: 44^{m}: 53.8^{s}$

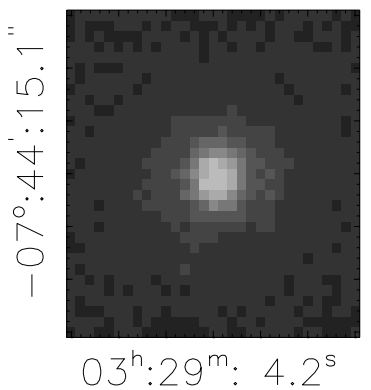

FIG. $8 f$

TABLE 2A

The SDSS Early-Type Galaxies Catalog: Observed Parameters

\begin{tabular}{|c|c|c|c|c|c|c|c|c|c|c|c|c|}
\hline $\begin{array}{l}\text { R.A. (J2000.0) } \\
\text { (deg) }\end{array}$ & $\begin{array}{c}\text { Decl. (J2000.0) } \\
\text { (deg) }\end{array}$ & $z$ & $\delta z$ & $\mathrm{~S} / \mathrm{N}$ & $\begin{array}{c}r_{\mathrm{dev}} \\
(\operatorname{arcsec})\end{array}$ & $\begin{array}{c}\delta r_{\mathrm{dev}} \\
(\operatorname{arcsec})\end{array}$ & $\begin{array}{c}m_{\mathrm{dev}} \\
(\mathrm{mag})\end{array}$ & $\begin{array}{c}\delta m_{\mathrm{dev}-\text { photo }} \\
\text { (mag) }\end{array}$ & $\begin{array}{l}m_{\mathrm{mod}} \\
(\mathrm{mag})\end{array}$ & $\begin{array}{l}\delta m_{\text {mod-photo }} \\
\text { (mag) }\end{array}$ & $b / a$ & $\delta b / a$ \\
\hline 113.810738 & 36.307522 & 0.25100 & 0.00009 & 12 & 3.45 & 0.15 & 17.234 & 0.009 & 17.234 & 0.009 & 0.82 & 0.03 \\
\hline 115.321098 & 37.511093 & 0.08513 & 0.00008 & 19 & 2.55 & 0.04 & 16.400 & 0.009 & 16.400 & 0.009 & 0.99 & 0.01 \\
\hline 114.201271 & 36.971313 & 0.07832 & 0.00008 & 15 & 2.16 & 0.05 & 17.202 & 0.009 & 17.202 & 0.009 & 0.71 & 0.02 \\
\hline 114.306282 & 37.111927 & 0.13049 & 0.00008 & 20 & 2.48 & 0.05 & 16.828 & 0.008 & 16.828 & 0.008 & 0.68 & 0.01 \\
\hline 113.294510 & 36.370544 & 0.21756 & 0.00007 & 13 & 2.18 & 0.08 & 17.451 & 0.008 & 17.451 & 0.008 & 0.80 & 0.03 \\
\hline
\end{tabular}

NoTES.-Table 2 is presented in its entirety in the electronic edition of the Astronomical Journal. A portion is shown here for guidance regarding its form and content. This table lists the $r^{*}$-band photometric parameters. Similar tables showing parameters in the $g^{*}, i^{*}$, and $z^{*}$ bands are also available in the electronic edition. 


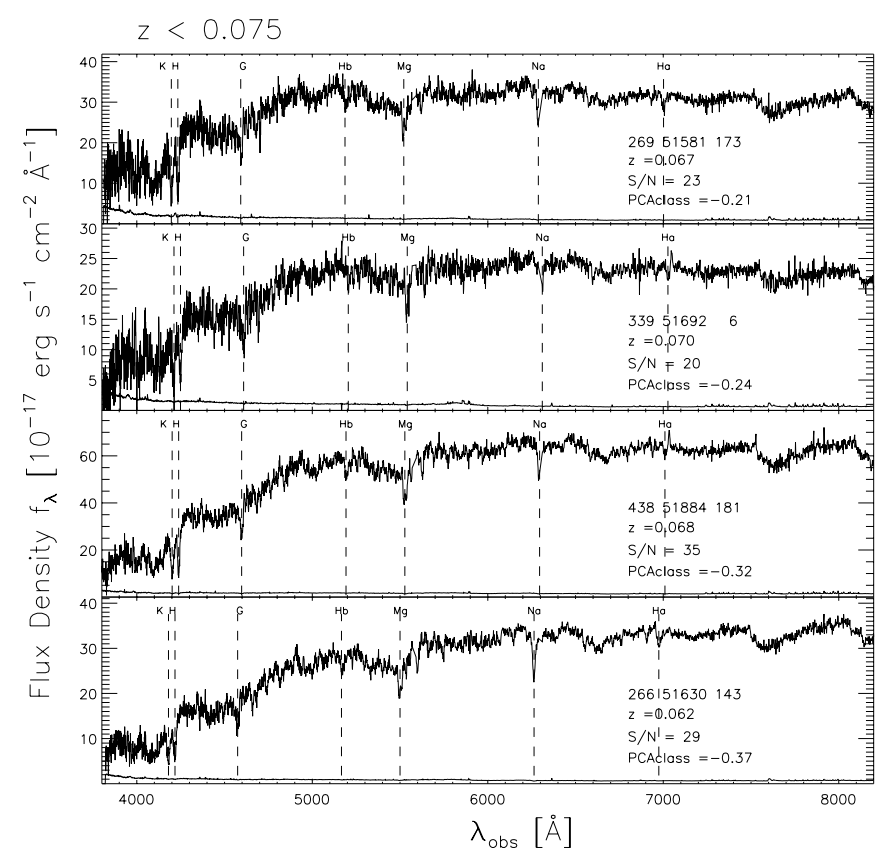

FIG. $9 a$

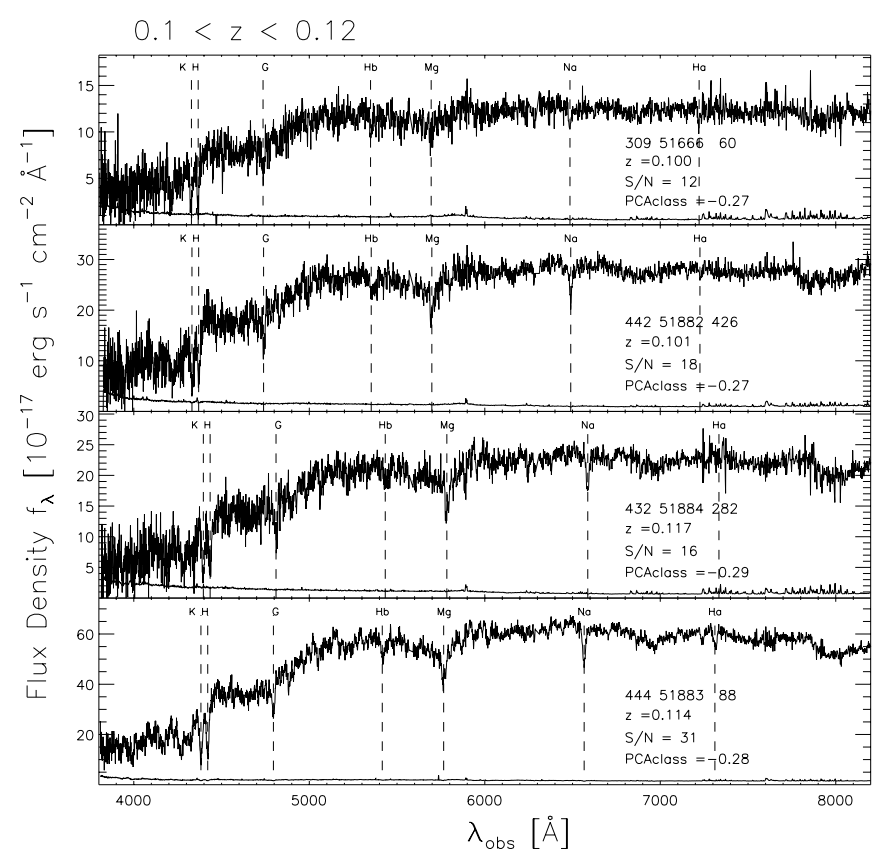

FIG. $9 c$

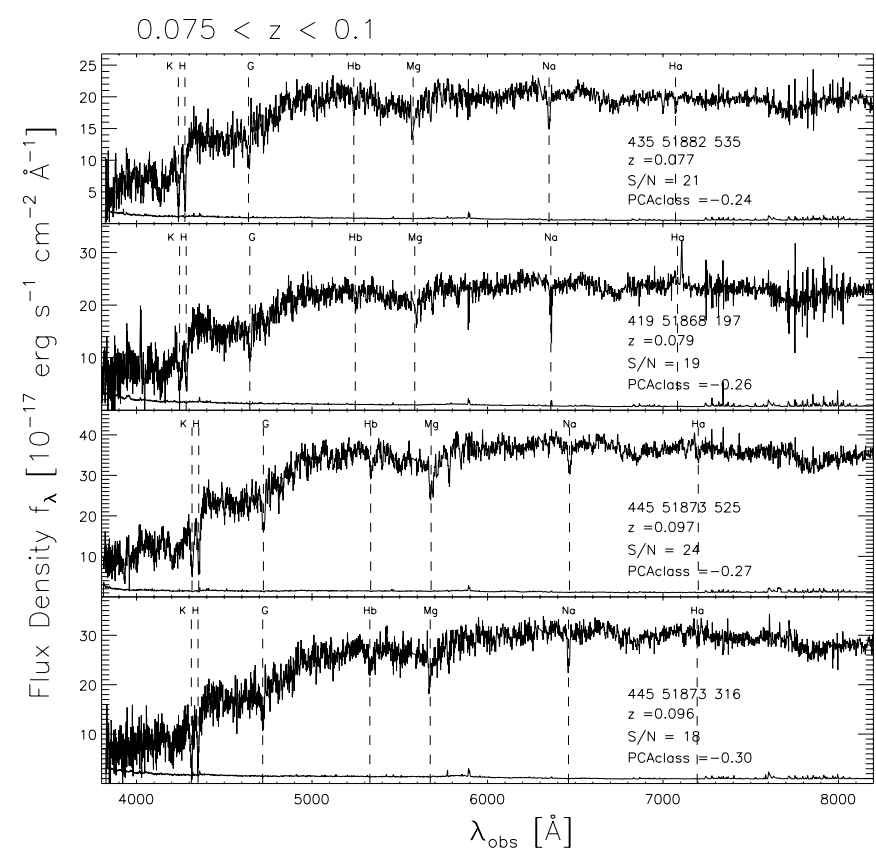

FIG. $9 b$

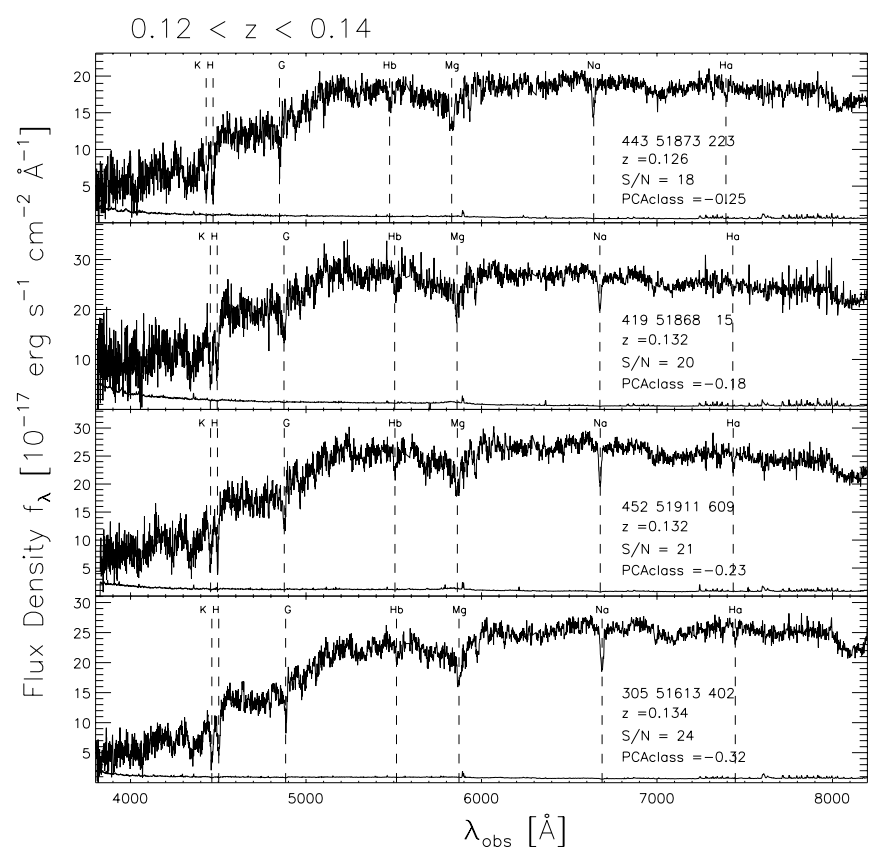

FIG. $9 d$

Fig. 9.- Spectra of galaxies that lie along the diagonals of Figs. 7 and 8. Text in each panel gives the plate number, MJD, and fiber identification, the redshift $z$, the $\mathrm{S} / \mathrm{N}$, and the PCA classification number. Some common absorption features are also indicated. The line along the bottom of each panel shows the error in the flux density in each pixel. Galaxies span the redshift range $(a) z<0.075,(b) 0.075<z<0.1,(c) 0.1<z<0.12,(d) 0.12<z<0.14,(e) 0.14<z<0.18$, and $(f) z>0.18$.

R.A., decl., aperture-corrected velocity dispersion $\log \sigma$ and its error estimate, the physical size $\log R_{o}$ and error estimate, the surface brightness $\mu_{o}$ and error estimate, the absolute magnitude $M_{\mathrm{dev}}$ and error estimate, the absolute model magnitude $M_{\bmod }$ and error estimate, the $K$-correction, and the number of neighbors that we use as an estimate of the local density. (The errors in $M_{\mathrm{dev}}$ and $M_{\text {model }}$ listed in this table were derived from the estimates $\delta m_{\text {photo }}$ of the previous table. As discussed in Appendix D, they are also the appropriate estimates for the error on $m_{\mathrm{dev}}$ and $m_{\text {mod. }}$.)
Some properties of our $r^{*}$ sample as a function of redshift $z$ are shown in Figure 12. The panels show the $K$-corrected absolute magnitude $M$, the $K$-corrected effective surface brightness $\mu_{o}$, also corrected for cosmological surface brightness dimming, the effective circular radius $R_{o}$ in $h_{70}^{-1} \mathrm{kpc}$, corrected to a standard rest-frame wavelength, the aperture corrected velocity dispersion $\sigma$ in $\mathrm{km} \mathrm{s}^{-1}$, and two quantities that are related to an effective mass and density, all plotted as a function of redshift. 


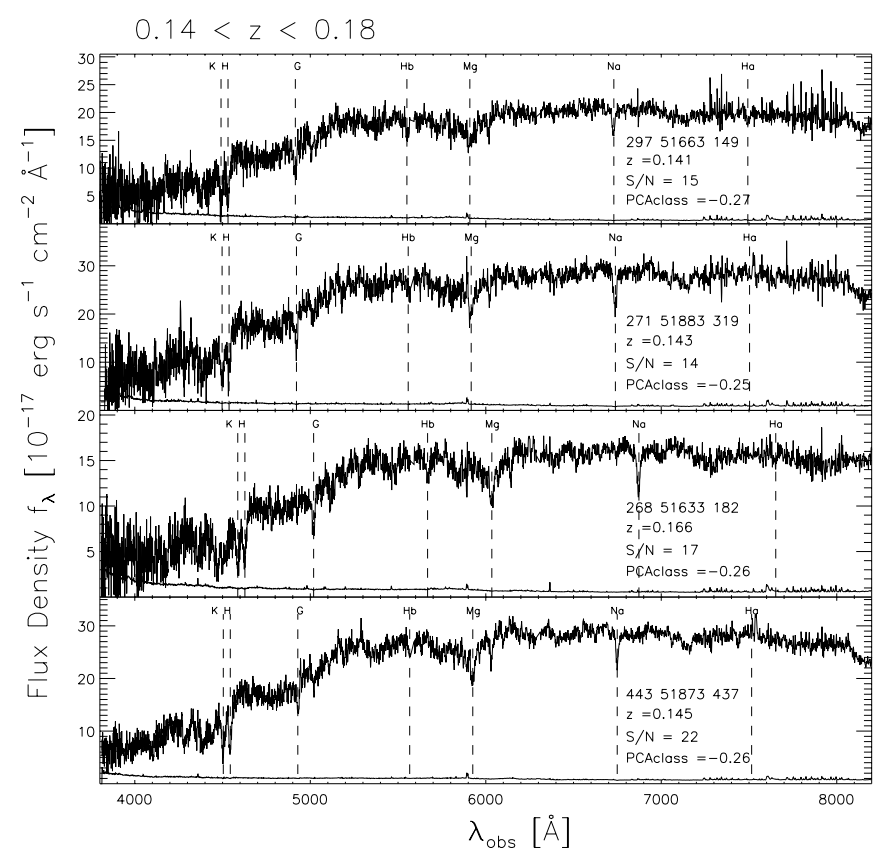

FIG. $9 e$

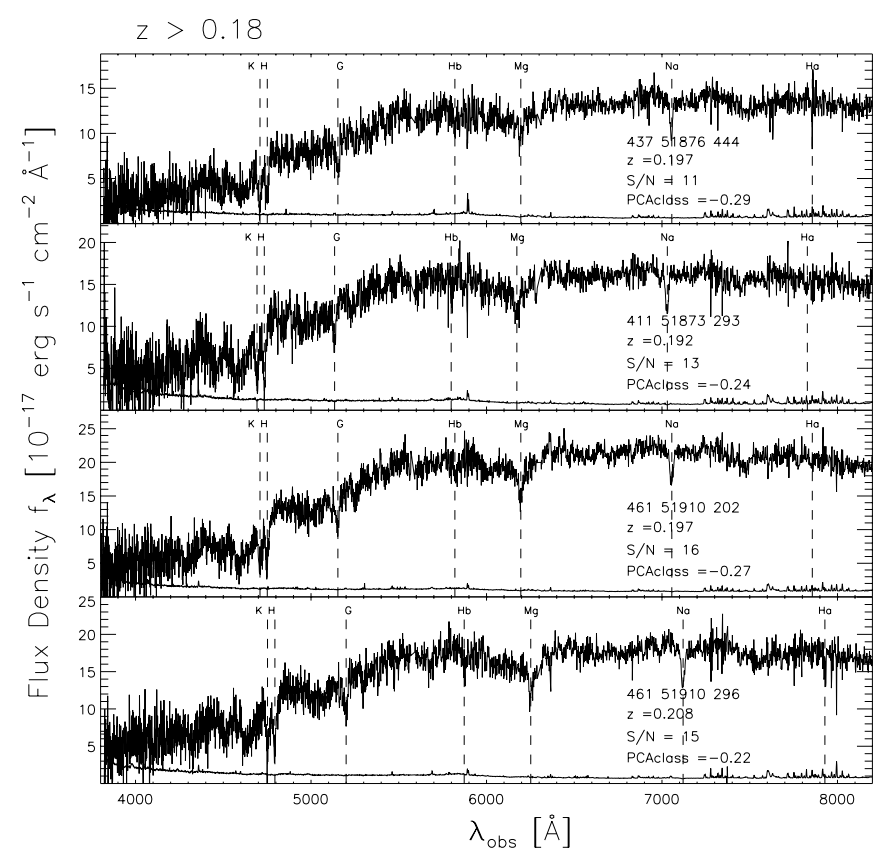

FIG. $9 f$

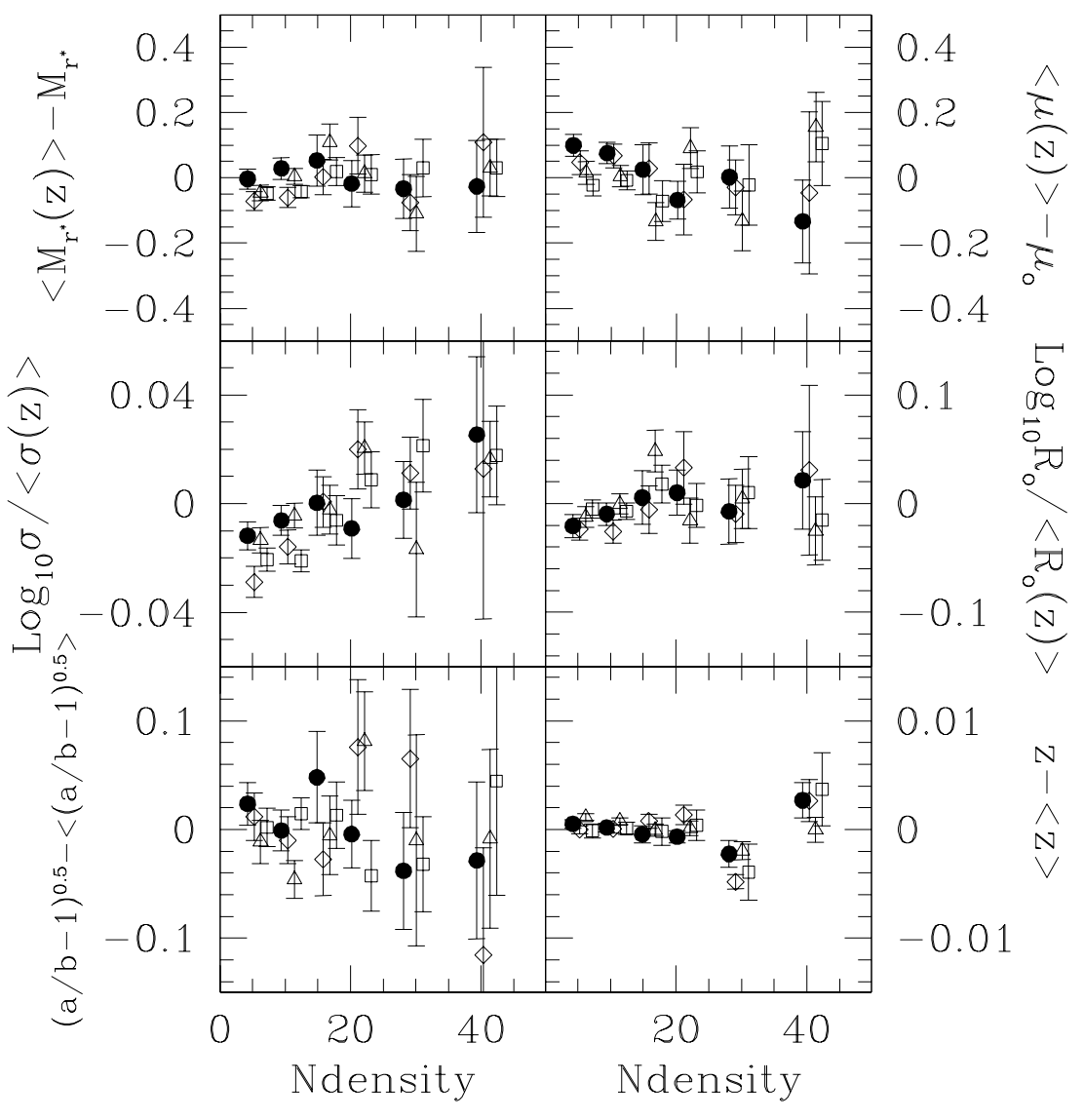

FIG. 10.-Luminosities, surface brightnesses, velocity dispersions, sizes, axis ratios, and mean redshifts, as a function of nearby early-type neighbors. The different symbols for each bin in density show averages over galaxies in different redshift bins: circles, diamonds, triangles, and squares are for galaxies with redshifts in the range $0.075<z \leq 0.1,0.1<z \leq 0.12,0.12<z \leq 0.14$, and $0.14<z \leq 0.18$. Although the velocity dispersions appear to increase with increasing local density, the increase is small. 


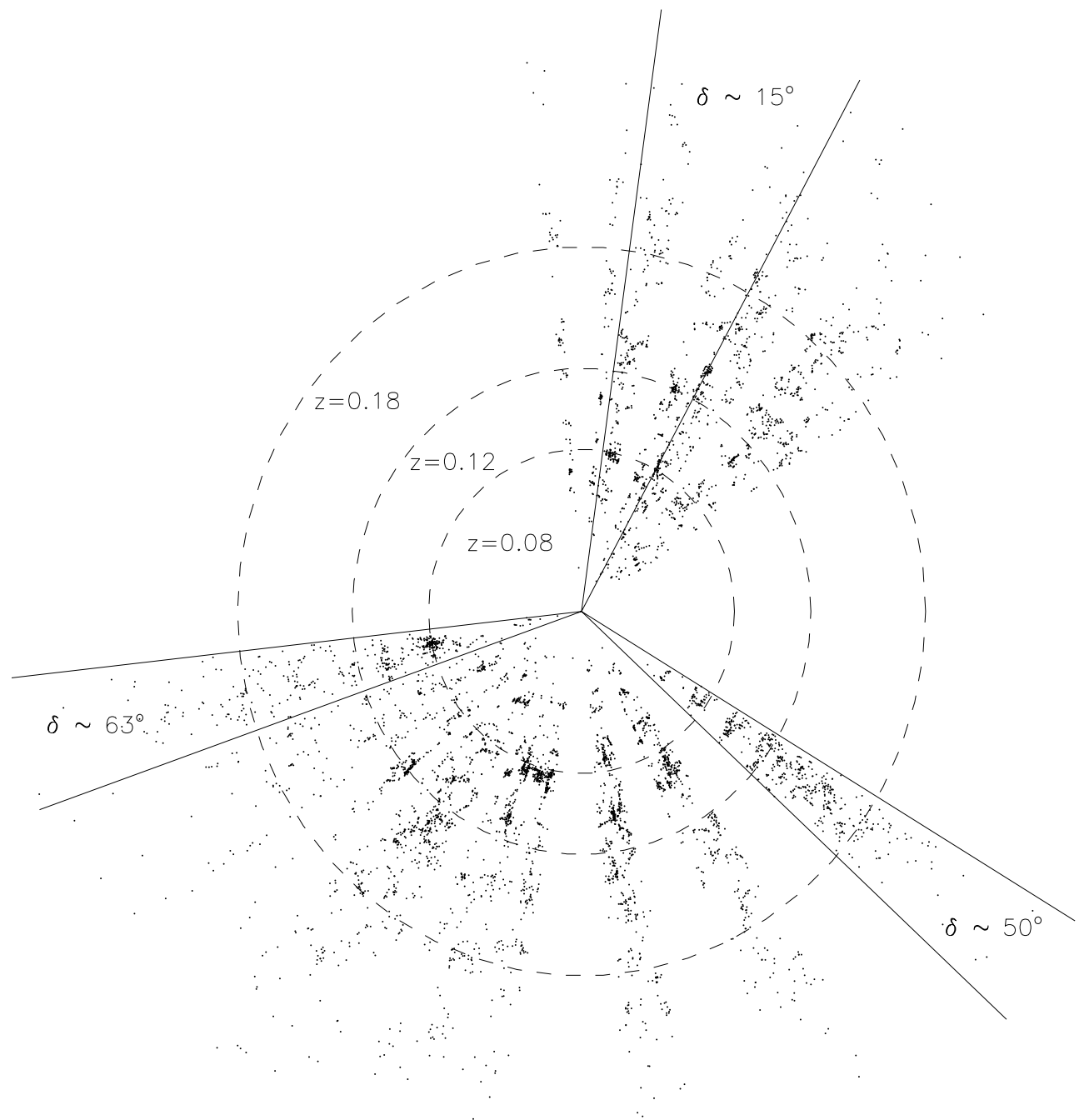

Fig. 11.-Pie-diagram distribution of our sample. Most of the sample is at low declination $\left(|\delta| \leq 2^{\circ}\right.$ ), but three wedges are at higher declinations (as indicated). Right ascension increases clockwise, with the zero at twelve o'clock.

The bold lines in the top left panel show the effect of the apparent magnitude cuts. There is, in addition, a cut at small velocity dispersion $\left(\sim 70 \mathrm{~km} \mathrm{~s}^{-1}\right)$ that, for our purposes here, is mostly irrelevant. The apparent magnitude cuts imply complex $z$-dependent cuts on the other parameters we observe. In the papers that follow, we attempt to account correctly for the selection effects that the magnitude cuts imply.

TABLE $3 \mathrm{~A}$

The SDSS Early-Type Galaxies Catalog: Physical Parameters

\begin{tabular}{|c|c|c|c|c|c|c|c|c|c|c|c|c|c|}
\hline $\begin{array}{l}\text { R.A. } \\
\text { (deg) }\end{array}$ & $\begin{array}{l}\text { Decl. } \\
\text { (deg) }\end{array}$ & $\begin{array}{c}V \\
(\mathrm{dex})\end{array}$ & $\begin{array}{c}\delta V \\
(\mathrm{dex})\end{array}$ & $\begin{array}{c}R \\
(\mathrm{dex})\end{array}$ & $\begin{array}{c}\delta R \\
(\mathrm{dex})\end{array}$ & $\begin{array}{c}\mu_{o} \\
(\mathrm{mag})\end{array}$ & $\begin{array}{c}\delta \mu_{o} \\
(\mathrm{mag})\end{array}$ & $\begin{array}{c}M_{\mathrm{dev}} \\
(\mathrm{mag})\end{array}$ & $\begin{array}{l}\delta M_{\mathrm{dev}} \\
(\mathrm{mag})\end{array}$ & $\begin{array}{l}M_{\text {mod }} \\
(\mathrm{mag})\end{array}$ & $\begin{array}{c}\delta M_{\text {mod }} \\
(\mathrm{mag})\end{array}$ & $\begin{array}{c}K \text {-corr } \\
(\mathrm{mag})\end{array}$ & Nden \\
\hline 113.810738 & 36.307522 & 2.501 & 0.034 & 0.979 & 0.020 & 19.873 & 0.060 & -23.587 & 0.033 & -23.587 & 0.033 & 0.309 & 4 \\
\hline 115.321098 & 37.511093 & 2.133 & 0.029 & 0.600 & 0.007 & 19.918 & 0.020 & -21.648 & 0.018 & -21.648 & 0.018 & 0.104 & 4 \\
\hline 114.201271 & 36.971313 & 2.055 & 0.045 & 0.414 & 0.012 & 19.993 & 0.034 & -20.643 & 0.021 & -20.643 & 0.021 & 0.093 & 3 \\
\hline 114.306282 & 37.111927 & 2.363 & 0.022 & 0.664 & 0.010 & 19.620 & 0.030 & -22.268 & 0.019 & -22.268 & 0.019 & 0.159 & 2 \\
\hline 113.294510 & 36.370544 & 2.307 & 0.040 & 0.871 & 0.017 & 19.944 & 0.052 & -22.978 & 0.029 & -22.978 & 0.029 & 0.268 & 4 \\
\hline
\end{tabular}

NoTES.-Table 3 is presented in its entirety in the electronic edition of the Astronomical Journal. A portion is shown here for guidance regarding its form and content. R.A. and Decl. are in J2000.0. We have set $V=\log \sigma$ with $\sigma$ in $\mathrm{km} \mathrm{s}^{-1}, R=\log R_{o}$ with $R_{o}$ in $\mathrm{kpc} h_{70}^{-1}, K$-corr is the $K$-correction and Nden is the number of near neighbors that we use to estimate the local density. Objects for which the number of neighbors was not determined have been assigned Nden $=999$, and we have set Nden $=100$ even if the number of neighbors is larger than 100 . This table lists the $r^{*}$-band photometric parameters. Similar tables showing parameters in the $g^{*}, i^{*}$, and $z^{*}$ bands are also available in the electronic edition. 


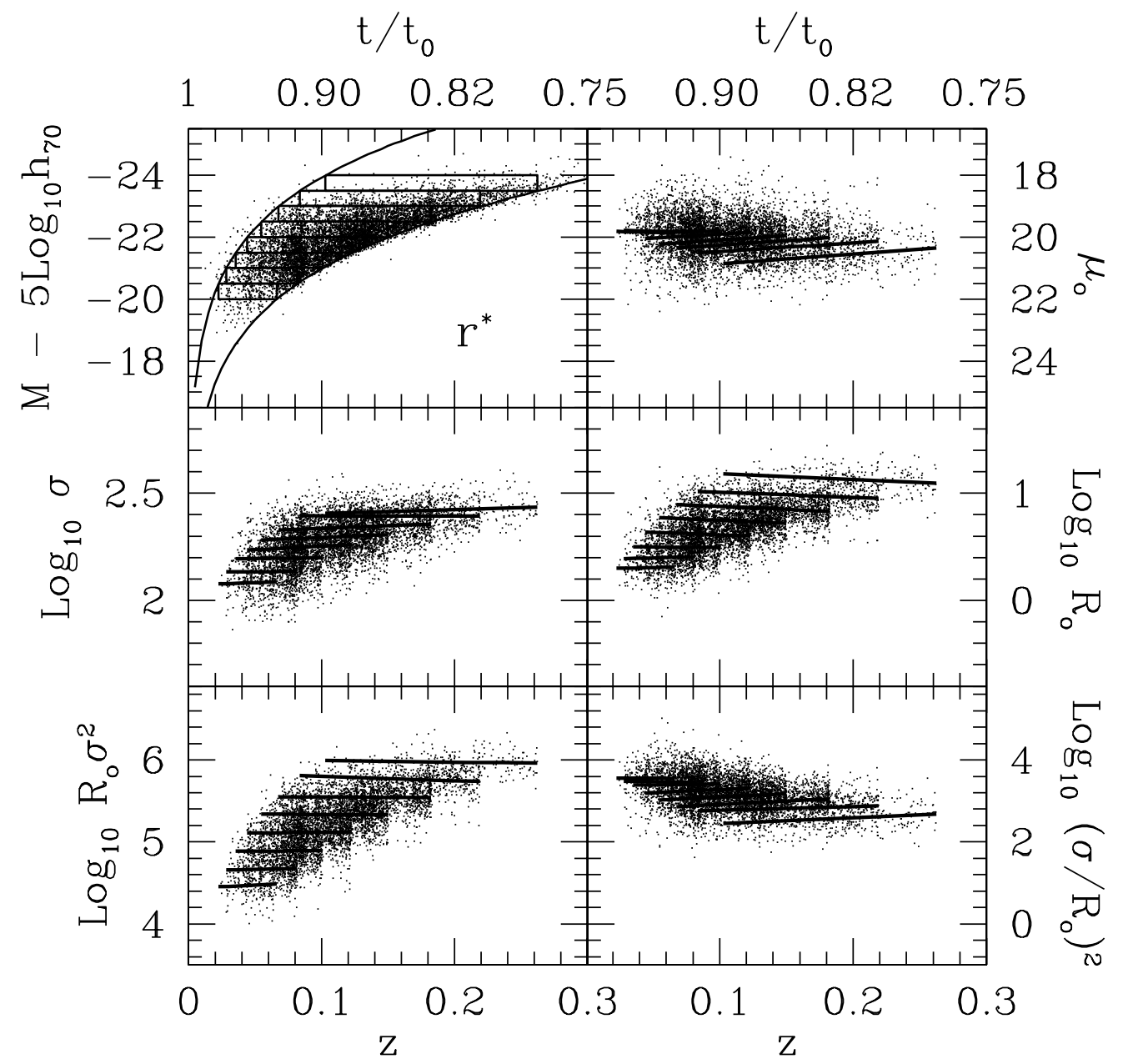

FIG. 12.- Surface brightnesses, velocity dispersions, sizes, masses, and densities of galaxies as a function of redshift, for a few bins in $r^{*}$ luminosity. Top left: Volume-limited catalogs that do not overlap in $r^{*}$ luminosity, dots in the other panels show the galaxies in the volume-limited subsamples defined by the top left panel, and solid lines show the mean trend with redshift in each subsample. Results in $g^{*}, i^{*}$, and $z^{*}$ are similar.

For small intervals in luminosity, our sample is complete over a reasonably large range in redshifts. To illustrate, the thin boxes in the top left panel show bins in absolute magnitude of width 0.5 mag over which the sample is complete. The solid lines in the other panels show how the median surface brightnesses, sizes, velocity dispersions, masses, and densities of galaxies in each $r^{*}$ luminosity bin change as a function of redshift. Although all these quantities depend on luminosity, the figure shows that, at fixed luminosity, there is some evidence for evolution: at fixed luminosity the average surface brightness is brightening. The size at fixed luminosity decreases at a rate that is about 5 times smaller than the rate of change of $\mu_{o}$. This suggests that it is the luminosities which are changing and not the sizes. (To see why, suppose that the average size at fixed absolute magnitude is $\left\langle\log \left[R_{o} / R_{*}(z)\right]\right\rangle=s\left[M-M_{*}(z)\right]$, where $R_{*}$ and $M_{*}$ are characteristic values, and $s$ is the slope of this mean relation. If the characteristic luminosity increases with $z$, but the characteristic size remains constant, $R *(z)=R *(0)$, and the slope of the relation also does not change, then the mean size at fixed $M$ decreases with $z$. The surface brightness is $\mu_{o} \propto M+5 \log R_{o}$, so the mean $\mu_{o}$ at fixed $M$ changes 5 times faster than the mean $R_{o}$, at fixed luminosity. This argument is also relevant to Figures 5 and 6, since those plots use $r_{\mathrm{dev}}$, which differs from $R_{o}$ by two small correction factors [c.f. § 3.2.1].) In Paper II, we argue that this trend is qualitatively consistent with that expected of a passively evolving population.

\section{SUMMARY}

This is the first of four papers that study the properties of early-type galaxies at relatively low redshifts $(z \leq 0.3)$. This paper describes how we selected the sample from the SDSS database using objective criteria and present the measured photometric and spectroscopic parameters for $\sim 9000$ earlytype galaxies (Table 2). The database contains the redshift of these galaxies, as well as reliable measurements of a number of photometric properties (luminosities, sizes, surface brightnesses, colors) in the $g^{*}, r^{*}, i^{*}$, and $z^{*}$ bands. (Data in the $u^{*}$ band is also available, but, because it is noisier, we did not use it here.)

The galaxies in our sample span the redshift range $0.01 \leq z<0.3$. Therefore, differences between observed and rest-frame wavelengths are not negligible. We discussed a number of methods for estimating the appropriate $K$ correction, which must be applied to obtain luminosities that sample the same rest-frame wavelength range (Appendix A). Since the half-light radii also depend on wavelength (Fig. 3), we also corrected these to sizes at fixed rest-frame 
wavelengths. Finally, we estimated the internal velocity dispersions of these galaxies (Appendix B), which, following common practice, we corrected to an aperture of $r_{e} / 8$ (c.f. eq. [1]). The resulting catalog listing the rest-frame photometric and spectroscopic parameters of $\sim 9000$ early-type galaxies (Table 3) is useful for measuring correlations between these various observables, such as the FaberJackson $L-\sigma$ relation, the Kormendy $R_{o}-I_{o}$ relation, the $L$ $R_{o}$ relation, the fundamental plane $R_{0}-I_{o}-\sigma$ relation, and the color-magnitude relation.

The sample is essentially magnitude-limited (Table 1, Fig. 2 ), and the galaxies in it populate a range of environments (Fig. 11). Therefore, the sample can be used for studying how the correlations discussed above depend on environment and redshift. For instance, Figure 12 shows evidence for weak evolution in the luminosities, and Figure 10 shows evidence for some weak environmental dependences. These will be quantified in Papers II and III.

Neither the $K$-corrections nor the aperture corrections we apply to the velocity dispersions are ideal. By the time SDSS is complete, the uncertainties in the $K$-corrections, which prevent us at the present time from making precise quantitative statements about the evolution of the luminosities and colors, will be better understood. In addition, the size of the sample will have increased by more than an order of magnitude. This larger sample will allow us to use the data directly to estimate the correct aperture correction that should be applied to the velocity dispersions. This is discussed more fully in Appendix C.

We would like to thank S. Charlot for making his stellar population synthesis predictions for the SDSS filters available to the collaboration and N. Benitez for making his package available. We thank M. Strauss for helpful discussions. Funding for the creation and distribution of the SDSS Archive has been provided by the Alfred P. Sloan Foundation, the Participating Institutions, the National Aeronautics and Space Administration, the National Science Foundation, the US Department of Energy, the Japanese Monbukagakusho, and the Max Planck Society. The SDSS Web site is http://www.sdss.org/. The SDSS is managed by the Astrophysical Research Consortium for the Participating Institutions. The Participating Institutions are the University of Chicago, Fermilab, the Institute for Advanced Study, the Japan Participation Group, Johns Hopkins University, Los Alamos National Laboratory, the Max-Planck-Institute for Astronomy, the Max-PlanckInstitute for Astrophysics, New Mexico State University, University of Pittsburgh, Princeton University, the US Naval Observatory, and the University of Washington.

\section{APPENDIX A}

\section{$K$-CORRECTIONS}

When converting the observed apparent magnitude to the rest-frame absolute magnitude of an object, we must account for the fact that the SDSS filters measure the light from a fixed spectral range in the observer's rest frame; therefore, they measure different parts of the rest-frame spectrum of galaxies at different redshifts. Correcting for this is known as the $K$-correction. One way to make this correction is to assume that all the galaxies at a given redshift are similar and to use an empirically determined template spectrum, measured from a few accurately measured spectra, to estimate the $K$-correction. Using a mean color to estimate the $K$-correction is not ideal. When the survey is closer to completion, it should become possible to make this correction on an object-by-object basis.

Empirically determined template spectra for early-type galaxies at low redshifts exist (e.g., Coleman et al. 1980; Fukugita, Shimasaku, \& Ichikawa 1995). [We used N. Benitez's Bayesian Photometric Redshift package (Benitez $2000)$ to derive $K$-corrections for the Coleman et al. earlytype galaxy template in the SDSS passbands.] If we were certain that evolution effects were not important, then these empirically determined $K$-corrections would allow us to work out the $K$-corrections we should apply to the highredshift population. However, if the stars in early-type galaxies formed at approximately the same time and if the mass in the galaxies has remained constant, so the evolution is entirely due to the passive aging of the stellar population, then the mass-to-light ratio of early-type galaxies is expected to vary approximately as $M / L \propto\left(t-t_{\text {form }}\right)^{-0.6}$ (e.g., Tinsley $\&$ Gunn 1976), with the precise scaling being different in different bands. Because our sample spans a relatively large range in redshift, we may be sensitive to the effects of this passive evolution. In addition, because the sample is large, we may be able to measure, and hence be sensitive to, even a relatively small amount of evolution. For this reason, it would be nice to have a prescription for making $K$-corrections that accounts for evolution. Without empirically determined templates for this evolution, we must use stellar population synthesis models to estimate this evolution, and so determine $K$-corrections for different bands.

As a first example, we chose a Bruzual \& Charlot (2003) model for a $10^{11} M_{\odot}$ object that formed its stars with the universal IMF given by Kroupa (2001) in a single solar metallicity and abundance ratio burst 9 Gyr ago. We then recorded how its colors, as observed through the SDSS filters, changed as it was moved through redshift without altering its age. This provides what we will call the noevolution $K$-correction. Figure 13 shows a comparison of this with the empirical Coleman et al. (1980) nonevolving $K$ corrections. The two estimates are in good agreement in $g^{*}$ and $i^{*}$ out to $z \sim 0.3$. They differ substantially at higher redshifts, but this is not a concern because none of the galaxies in our sample are so distant. In $r^{*}$ and $z^{*}$, the two estimates agree only at $z \leq 0.15$. Therefore, quantitative estimates of evolution in luminosity and/or color will depend on which $K$-correction we use.

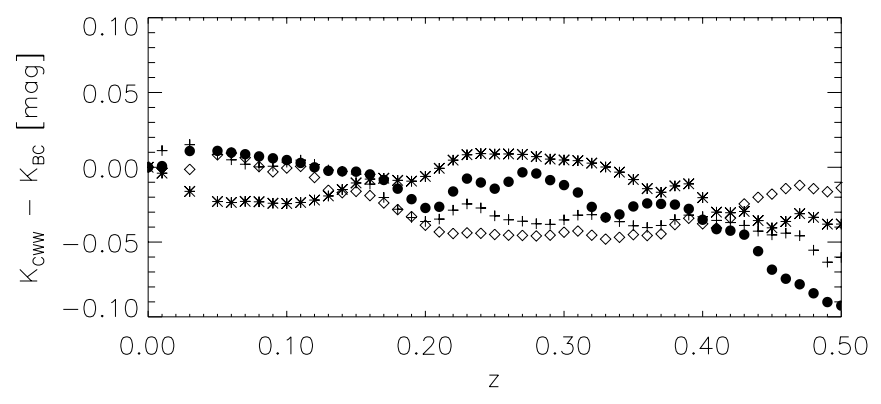

FIG. 13.-Difference between $K$-corrections based on two models (Coleman et al. 1980 and Bruzual \& Charlot 2003) of the SDSS colors of earlytype galaxies. Filled circles, crosses, stars, and diamonds are for the $g^{*}, r^{*}$, $i^{*}$, and $z^{*}$ bands. 


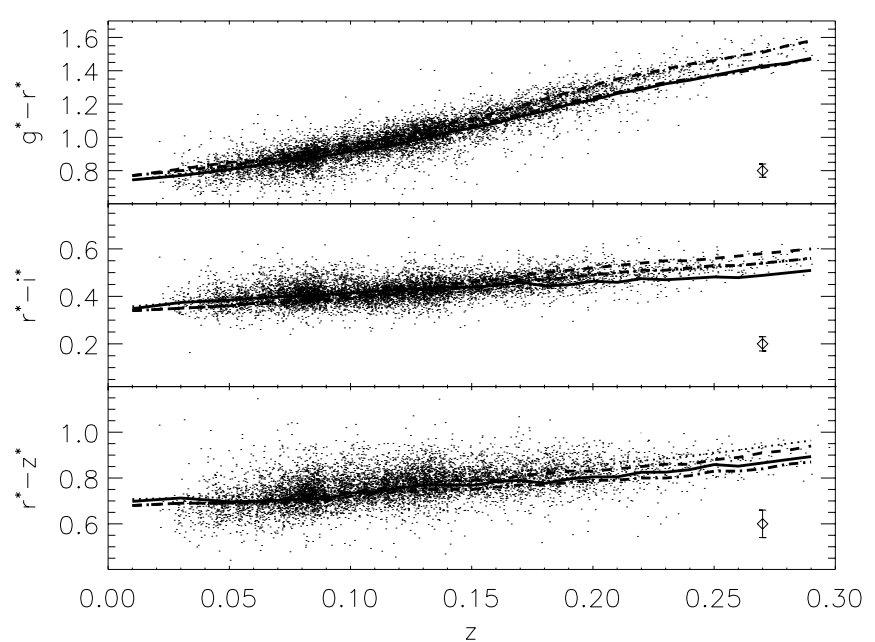

FIG. 14.-Apparent colors of the galaxies in our sample. In each panel, the dotted and solid lines show the nonevolving and evolving Coleman et al. templates, whereas the dashed and dot-dashed lines show Bruzual \& Charlot models. The top set of curves in each panel show what one expects to see if the intrinsic colors of galaxies at higher redshifts are the same as they are nearby, whereas the lower sets of curves show the predictions if the higher redshift population is slightly younger and so bluer. The magnitude limit of the sample makes it appear as though the no-evolution curves describe our data well. In the main text, we use the lower solid curve to make $K$-corrections to the observed magnitudes.

Figure 14 compares both sets of nonevolving templates with the observed colors of the galaxies in our sample. The upper set of curves in each panel show the colors associated with the nonevolving Coleman et al. (1980) template (dotted line) and the Bruzual \& Charlot (2003) no-evolution model (dashed line). The figure shows that both predictions for $g^{*}-r^{*}$ are similar, but that they are different for $r^{*}-i^{*}$ and $r^{*}-z^{*}$, with the differences increasing with redshift. [In both cases, we have shifted the predicted $g^{*}-r^{*}$ blueward by 0.08 mag at all $z$. Such an offset appears to be required for the SDSS photometric calibrations in Stoughton et al. (2002) that we use here (also see Eisenstein et al. 2001 and Strauss et al. 2002), although the reason for it is not understood.]

We argue in Papers II, III, and IV that the luminosities, colors, and chemical abundance ratios of these objects show evidence for passive evolution: the higher redshift population appears to be slightly younger. Therefore, our next step is to include the effects of evolution. Because the predicted observed colors at redshift zero are in good agreement with our data, we took the same Bruzual \& Charlot (2003) model, but this time we recorded how its rest-frame colors evolve with redshift and then computed what these evolved (i.e., $z$-dependent) colors look like when observed in the SDSS filters. These evolving colors are shown as the dotdashed lines in Figure 14. In an attempt to include evolution in the Coleman et al. (CWW) templates, we set $K_{\mathrm{CWW}}^{\mathrm{evol}}(z)=K_{\mathrm{CWW}}^{\mathrm{no}-\mathrm{ev}}(z)+K_{\mathrm{BC}}^{\mathrm{evol}}(z)-K_{\mathrm{BC}}^{\mathrm{no}}(z)$. The $\quad$ lower solid lines in each panel of Figure 14 show the observed $g^{*}-r^{*}, r^{*}-i^{*}$, and $r^{*}-z^{*}$ colors associated with these evolving models [and again, the predicted $g^{*}-r^{*}$ curves have been shifted blueward (downward) by 0.08 mag at all $z$ ]. Comparing the evolving Bruzual-Charlot and the Coleman et al. colors with the upper set of no-evolution curves shows the evolution toward the blue at high redshift. Although the data appear to be very well fitted by the no-evolution curves, this agreement is slightly misleading. More luminous galaxies tend to be redder. As a consequence, a magnitude-

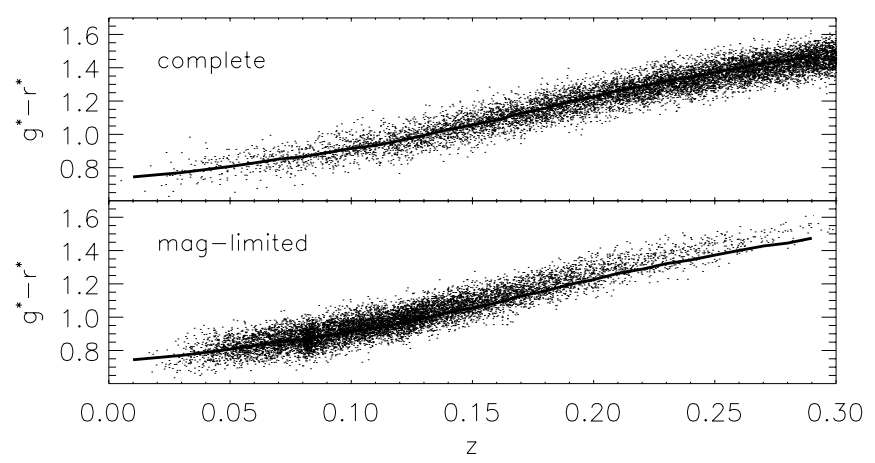

FIG. 15.-Apparent $g^{*}-r^{*}$ colors of simulated galaxies in mock catalogs of a passively evolving population; the galaxies at higher redshift are younger and, in their rest-frame, bluer. Top: Expected distribution of observed colors if there were no magnitude limit; bottom: effect of imposing the same magnitude limit as in our SDSS sample. Solid curves (same in both panels) show the trend of observed color with redshift of this evolving population. Although the smooth curve describes the complete catalog well, it is substantially bluer than the subset of objects that are included in the magnitude-limited sample. The difference between the curves and our magnitude-limited mock catalogs is similar to that between the curves and the data (see Fig. 14), suggesting that the colors of the galaxies in our data are evolving similarly to how we assumed in our simulations.

limited catalog contains only the redder objects of the higher redshift population. A curve that describes the colors of the population as a whole will therefore appear to be biased blue.

Figure 15 shows this explicitly. The two panels were constructed by making mock catalogs of a passively evolving population (i.e., the higher redshift population is brighter and bluer) in which our estimates of the correlation between colors, luminosities, velocity dispersions, and color and luminosity evolution were included (see Appendix A in Paper II). The top panel shows the distribution of observed colors if there was no magnitude limit, and the bottom panel shows the observed colors of a magnitude-limited sample. The solid curve, the same in both panels, is the predicted trend of color with redshift that we use to make our $K$ corrections; i.e., $K_{\mathrm{CWW}}^{\mathrm{evol}}(z)$. Note that although it describes the complete simulations well, it is bluer than the higher redshift galaxies in the magnitude-limited sample. Comparison with the previous figure shows that the difference here is similar to that seen in the real data, suggesting that our $K$-corrections and evolution estimates of the mean of the population are self-consistent.

Of course, if we do not observe the mean of the highredshift population, but only the redder fraction, then we must decide whether it is realistic to use a $K$-correction that has been constructed to fit the truly typical galaxy at each redshift. For example, if color is an indicator of age and/or metallicity, then the results above suggest that our sample contains the oldest and/or most metal-rich part of the highredshift population. If the objects that satisfy our apparent magnitude limit are, in fact, older than the typical highredshift galaxy, then it may be that those objects are similar in age to the average object at lower redshifts in our sample. If so, then we are better off using a nonevolving $K$ correction even though the higher redshift sample as a whole is younger. None of the results presented in the main text change drastically if we use nonevolving rather than evolving $K$-corrections.

To decide which $K$-corrections to use, we computed the color-magnitude and color- $\sigma$ relations discussed in Paper 
IV using both the Coleman et al. (1980) template and the Bruzual \& Charlot (2003) models. The slopes of the mean relations, and the scatter around the mean, remained approximately the same for both $K$-corrections, so we have chosen to not show them here. This suggests that our ignorance of the true $K$-correction does not strongly compromise our conclusions about how color correlates with magnitude and velocity dispersion. Conclusions about evolution, however, do depend on the $K$-correction.

The color- $\sigma$ relations constructed using either $K_{\mathrm{BC}}^{\mathrm{evol}}$ or $K_{\mathrm{CWW}}^{\text {evol }}$ show evidence for evolution. However, $K_{\mathrm{BC}}^{\text {evol }}$ yields evolution in $g^{*}-r^{*}$ of $0.04 \mathrm{mag}$, and in $r^{*}-i^{*}$ of $0.07 \mathrm{mag}$, whereas $K_{\mathrm{CWW}}^{\text {evol }}$ has changes of 0.07 and 0.03 , respectively. Thus, $K_{\mathrm{BC}}^{\text {evol }}$ suggests that the evolution in $r^{*}-i^{*}$ is larger than in $g^{*}-r^{*}$. This is not the expected trend; the $g^{*}-r^{*}$ and $r^{*}-i^{*}$ wavelength baselines are about the same, so one expects more of the evolution to come in at the bluer color. Using $K_{\mathrm{CWW}}^{\mathrm{evol}}$ instead suggests that most of the evolution is in $g^{*}-r^{*}$, which is more in line with expectations.

We also tried $K$-corrections from Fukugita et al. (1995). At low redshifts, the predicted early-type colors are redder than those in our sample, the predicted S0 colors are bluer, and the differences depend on redshift. A straight average of the two is an improvement, although the resulting lowredshift $g^{*}-r^{*}$ is red by 0.05 mag. If we shift by this amount to improve the agreement at low redshifts, then the observed $g^{*}-r^{*}$ colors at $z=0.25$ are redder than the predicted no evolution curves by about $0.2 \mathrm{mag}$. This is larger than the offset we expect for the selection effect introduced by the magnitude limit, so we decided against presenting further results from these $K$-corrections.

\section{APPENDIX B}

\section{VELOCITY DISPERSION: METHODS AND MEASUREMENTS}

This Appendix describes how we estimated the line-ofsight velocity dispersions $\sigma$ for the sample of galaxies selected for this paper. Estimates of $\sigma$ are limited by the instrumental dispersion and resolution. Recall that the instrumental dispersion of the SDSS spectrograph is $69 \mathrm{~km}$ $\mathrm{s}^{-1}$ per pixel, and the resolution is $\sim 90 \mathrm{~km} \mathrm{~s}^{-1}$. In addition, the instrumental dispersion may vary from pixel to pixel, and this can affect measurements of $\sigma$. These variations are estimated for each fiber by using arc lamp spectra (up to 16 lines in the range 3800-6170 $\AA$ and 39 lines between 5780 $9230 \AA$ ). An example of the variation in instrumental dispersion for a single fiber is shown in Figure 16. The figure shows that a simple linear fit provides a good description of this variation. This is true for almost all fibers and allows us to remove the bias such variations may introduce when estimating galaxy velocity dispersions.

A number of methods for making accurate and objective velocity dispersion measurements as have been developed (Sargent et al. 1977; Tonry \& Davis 1979; Franx et al. 1989; Bender 1990; Rix \& White 1992). These methods are all based on a comparison between the spectrum of the galaxy whose velocity dispersion is to be determined and a fiducial spectral template. This can either be the spectrum of an appropriate star, with spectral lines unresolved at the spectra resolution being used, or a combination of different stellar types, or a high $\mathrm{S} / \mathrm{N}$ spectrum of a galaxy with known

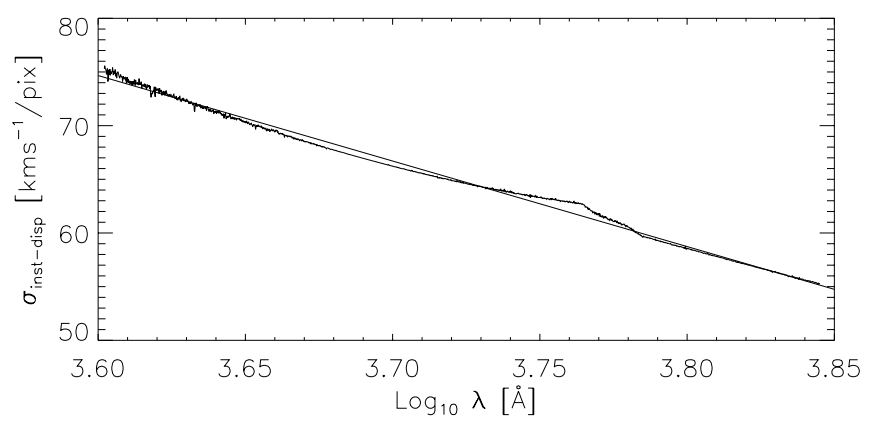

FIG. 16.-Variation of instrumental dispersion over the range in wavelengths used to measure velocity dispersions later in this paper. Solid line shows a linear fit.

velocity dispersion. In this work, we use SDSS spectra of 32 $\mathrm{K}$ and $\mathrm{G}$ giant stars in M67 as stellar templates.

Since different methods can give significantly different results, thereby introducing systematic biases especially for low $\mathrm{S} / \mathrm{N}$ spectra, we decided to use three different techniques for measuring the velocity dispersion. These are (1) the cross-correlation method (Tonry \& Davis 1979), (2) the Fourier-fitting method (Tonry \& Davis 1979; Franx et al. 1989; van der Marel \& Franx 1993), and (3) a modified version of the direct-fitting method (Burbidge, Burbidge, \& Fish 1961; Rix \& White 1992). Because a galaxy's spectrum is that of a mix of stars convolved with the distribution of velocities within the galaxy, Fourier space is the natural choice to estimate the velocity dispersions - the first two methods make use of this. However, there are several advantages to treating the problem entirely in pixel space. In particular, the effects of noise are much more easily incorporated in the pixel-space-based direct-fitting method. Because the $\mathrm{S} / \mathrm{N}$ of the SDSS spectra are relatively low, we assume that the observed absorption line profiles in earlytype galaxies are Gaussian (see Rix \& White 1992 and Bender, Saglia, \& Gerhard 1994 for a discussion of how to analyze the line profiles of high $\mathrm{S} / \mathrm{N}$ spectra in the case of asymmetric profiles).

It is well known that all three methods have their own particular biases, so that numerical simulations must be used to calibrate these biases. In our simulations, we chose a template stellar spectrum measured at high $\mathrm{S} / \mathrm{N}$, broadened it using a Gaussian with rms $\sigma_{\text {input }}$, added Gaussian noise, and compared the input velocity dispersion with the measured output value. The first broadening allows us to test how well the methods work as a function of velocity dispersion, and the addition of noise allows us to test how well the methods work as a function of $\mathrm{S} / \mathrm{N}$.

The best-case scenario is one in which there is no "template mismatch": the spectrum of the template star is exactly like that of the galaxy whose velocity dispersion one wishes to measure. Figure 17 shows the fraction of systematic bias associated with each of the different methods in this best-case scenario. Slightly more realistic simulations, using a combination of stellar spectra as templates, were also done. The results are similar to those shown in Figure 17. With the exception of the cross-correlation method at low $\left(\sigma<100 \mathrm{~km} \mathrm{~s}^{-1}\right)$ velocity dispersion, the systematic errors on the velocity dispersion measurements appear to be smaller than $\sim 3 \%$.

Although the systematics are small, note that the measured velocity dispersion is more biased at low velocity 


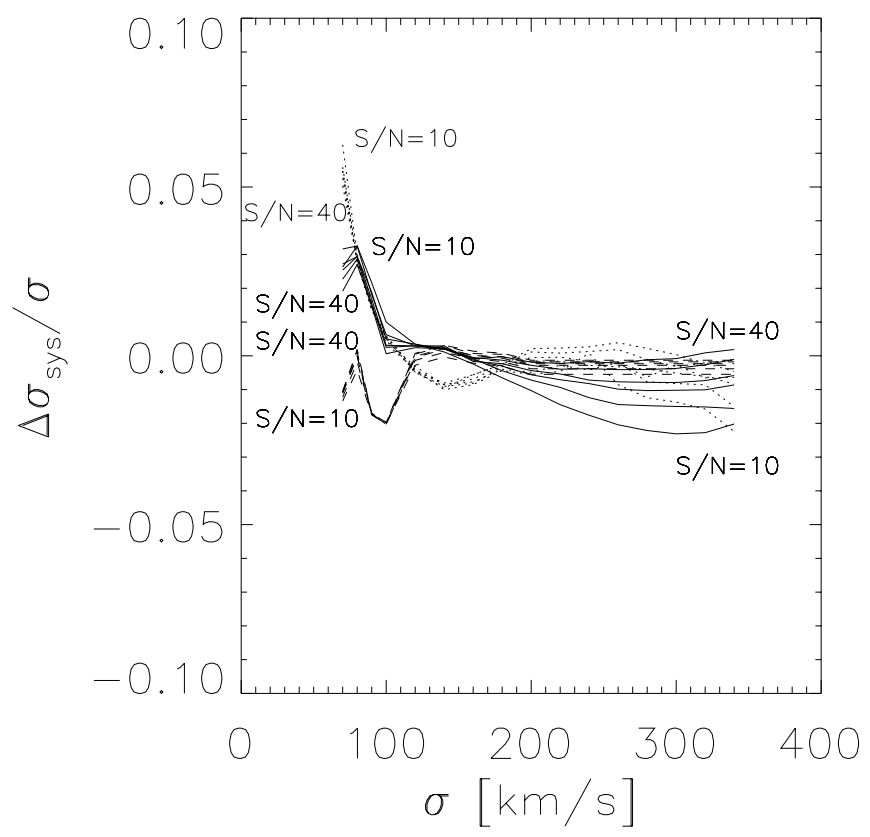

FIG. 17.-Systematic biases in the three methods used to estimate the velocity dispersion. Solid, dashed, and dotted lines show the biases in the Fourier-fitting, direct-fitting, and cross-correlation methods, as a function of velocity dispersion and $\mathrm{S} / \mathrm{N}$.

dispersions $\left(\sigma<100 \mathrm{~km} \mathrm{~s}^{-1}\right)$. For any given $\mathrm{S} / \mathrm{N}$ and resolution, there is a lower limit on the velocity dispersion measurable without introducing significant bias. Since the $\mathrm{S} / \mathrm{N}$ of the SDSS spectra is not very high (see, e.g., Fig. 18) and because the instrumental resolution is $\sim 90 \mathrm{~km} \mathrm{~s}^{-1}$, we chose $70 \mathrm{~km} \mathrm{~s}^{-1}$ as a lower limit. Figure 18 shows a comparison of the velocity dispersion estimates obtained from the three different methods for the galaxies in our sample. The median offsets are not statistically significant, and the rms scatter is $\sim 0.05$. On the other hand, the top panels suggest that the cross-correlation method sometimes underestimates the velocity dispersion, particularly at low $\mathrm{S} / \mathrm{N}$.

We evaluate the dependence of the velocity dispersion on the wavelength range by fitting the spectra in different intervals: $4000-5800 \AA$, which is the usual wavelength range used in the literature; $3900-5800 \AA$ to test the effect of including the $\mathrm{Ca} \mathrm{H}$ and $\mathrm{K}$ absorption lines (e.g., Kormendy 1982); $4000-6000 \AA$ to test the effect of including the $\mathrm{Na} \mathrm{D}$ line

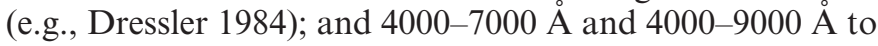
test the effect of including longer wavelengths. The velocity dispersion obtained with the $\mathrm{Ca} \mathrm{H}$ and $\mathrm{K}$ is $~ 2 \%$ larger than that obtained using the standard wavelength region 4000$5800 \AA$, and the rms difference between the three different methods increases to $\sim 7 \%$. Including the $\mathrm{Na} \mathrm{D}$ line increases the velocity dispersion by $\sim 3 \%$ but does not increase the scatter between the different methods. Using the wavelength range 4000-7000 $\AA$ only provides velocity dispersions that are $\sim 3 \%$ larger than the values obtained if only $4000-5800 \AA$ range is used. On the other hand, in this wavelength region, the different methods (and measurements from repeated observations) are in better agreement; the scatter is $\sim 8 \%$ smaller than in the $4000-5800 \AA$ region. In the range $4000-9000 \AA$, the velocity dispersion estimates increase by $\sim 7 \%$. This last effect is probably due to the presence of molecular bands in the spectra of early-type galaxies

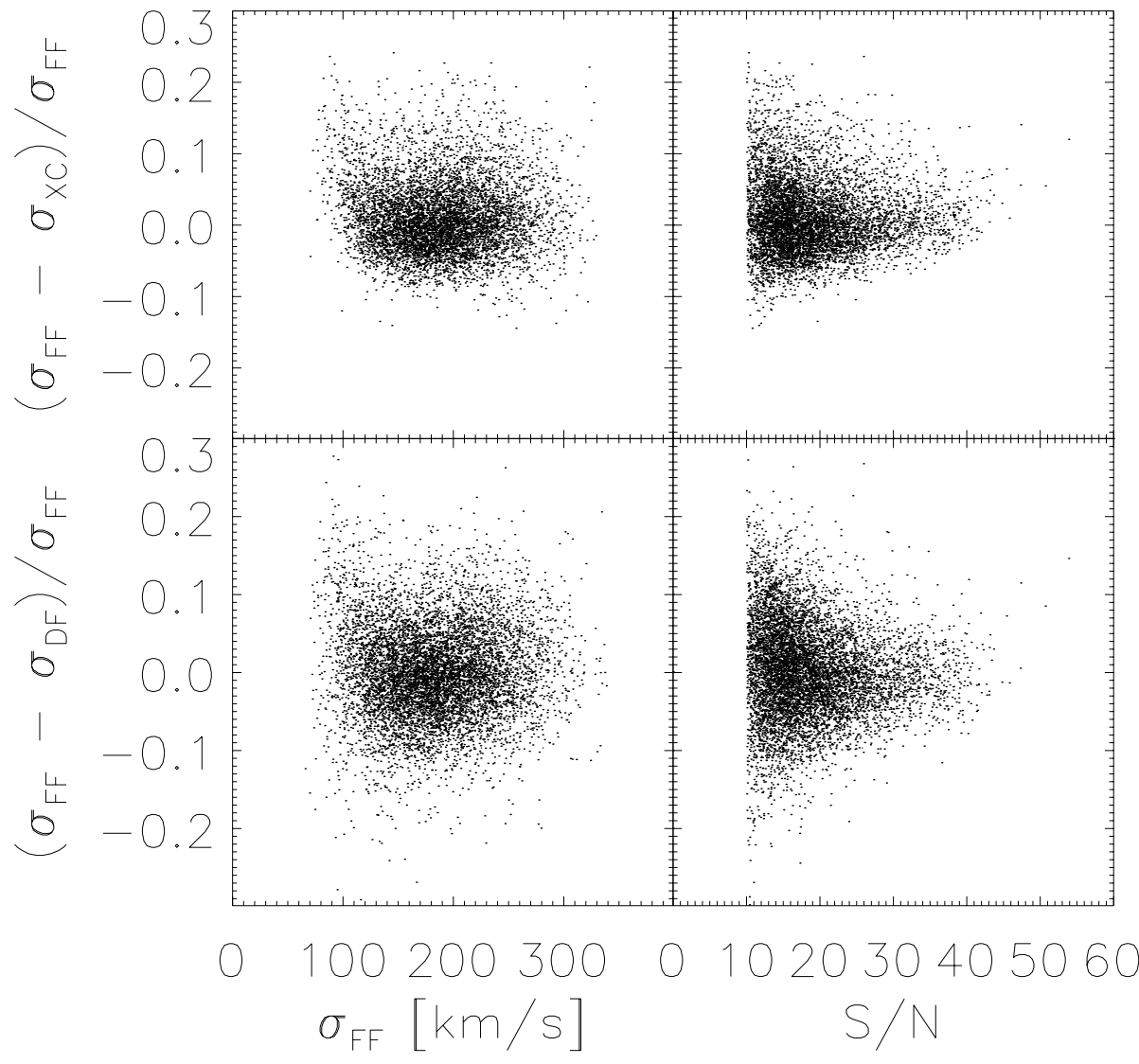

FIG. 18.-Comparison of the various methods used to estimate the velocity dispersions; the agreement is quite good, with a scatter of about $5 \%$. Most of our spectra have $\mathrm{S} / \mathrm{N} \sim 15$, with approximately exponential tails on either side of this mean value. 


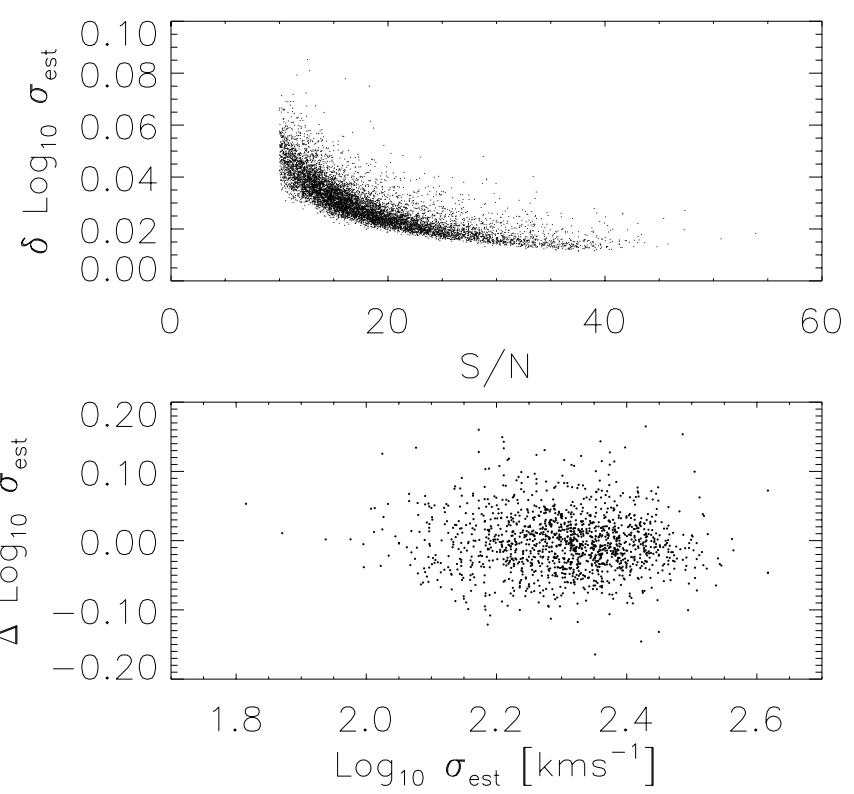

FIG. 19.-Distribution of errors as a function of $\mathrm{S} / \mathrm{N}(t o p)$ and comparison of estimates from repeated observations (bottom). Both panels suggest that, when the $\mathrm{S} / \mathrm{N} \geq 15$, then the typical error on an estimated velocity dispersion is $\delta \log \sigma<0.04$.

at long wavelengths (i.e., to the presence of cool stars). Furthermore, the scatter in this wavelength region increases dramatically $(\sim 15 \%)$. Presumably this is due to the presence of higher sky-line residuals and lower $\mathrm{S} / \mathrm{N}$.

The estimated velocity dispersions we use in the main text are obtained by fitting the wavelength range 4000-7000 $\AA$ and then using the average of the estimates provided by the Fourier-fitting and direct-fitting methods to define what we call $\sigma_{\text {est }}$. We do not use the cross-correlation estimate because of its behavior at low $\mathrm{S} / \mathrm{N}$ as discussed earlier.

The top panel of Figure 19 shows the distribution of the errors on the velocity dispersion as a function of the $\mathrm{S} / \mathrm{N}$ of the spectra. The errors for each method were computed by adding in quadrature the statistical error due to the noise properties of the spectrum, and the systematic error associated with the template and galaxy mismatches. The final error on $\sigma_{\text {est }}$ is computed by adding in quadrature the errors on the two estimates (i.e., the Fourier-fitting and direct-fitting) that we average. The resulting errors are in the range $0.02 \leq \delta \log \sigma \leq 0.06 \mathrm{dex}$, depending on the $\mathrm{S} / \mathrm{N}$ of the spectra, with a median value of 0.03 dex.

A few galaxies in our sample have been observed more than once. The bottom panel shows a comparison of the velocity dispersion estimates from multiple observations. The scatter between different measurements is $\sim 0.04$ dex, consistent with the amplitude of the errors on the measurements.

\section{APPENDIX C}

\section{VELOCITY DISPERSION: PROFILES AND APERTURE CORRECTIONS}

The SDSS spectra measure the light within a fixed aperture of radius 1 !. 5 . Therefore, the estimated velocity dispersions of more distant galaxies are affected by the motions of stars at larger physical radii than for similar galaxies that

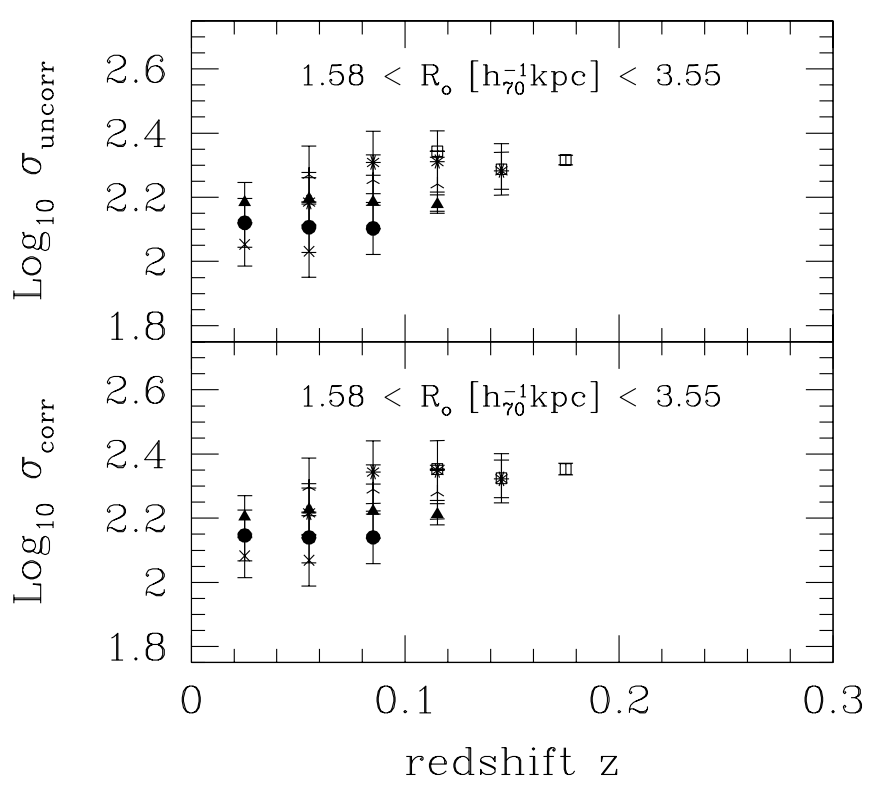

FIG. 20.-Velocity dispersions of galaxies as a function of redshift. Top: Estimated velocity dispersion; bottom: values after correcting the estimate as described in the main text (eq. [1]). Different symbols show the result of averaging over volume-limited subsamples (same as in Fig. 12) of galaxies having approximately the same luminosities and effective radii at each redshift. (Error bars show the rms scatter around this mean value.) The mean trends with redshift can be used to infer how, on average, the velocity dispersion changes with distance from the center of the galaxy, and how this change depends on luminosity and effective radius.

are nearby. If the velocity dispersions of early-type galaxies decrease with radius, then the estimated velocity dispersions (using a fixed aperture) of more distant galaxies will be systematically smaller than those of similar galaxies nearby.

We have not measured the velocity dispersion profiles $\sigma(r)$ of any of the galaxies in our sample, so we cannot correctly account for this effect. If we assume that the galaxies in our sample are similar to those for which velocity dispersion profiles have been measured, then we can use the

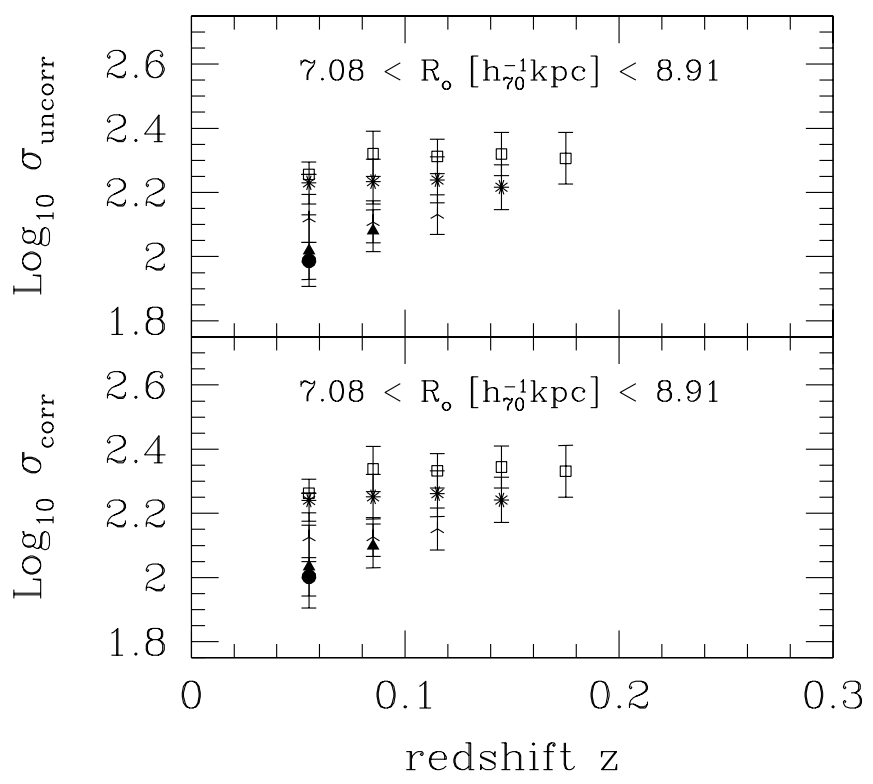

FIG. 21. - As for Fig. 20, but for galaxies with larger radii 
published $\sigma(r)$ curves to correct for this effect. This is what equation (1) in $\S 3.3 .1$ does.

An alternative procedure can be followed if evolution effects are not important for the velocity dispersions in our sample. To illustrate the procedure, the galaxies in each of the volume-limited subsamples shown in Figure 12 were further classified into small bins in effective physical radius (i.e., $R_{o}$ in $\mathrm{kpc} h^{-1}$, not $r_{o}$ in arcseconds). Figures 20-21 show the result of plotting the velocity dispersions of these galaxies versus their redshifts. Since the galaxies at low and high redshift are supposed to be similar, any trend with redshift can be used to infer and average velocity dispersion profile, and how the shape of this profile depends on luminosity and effective radius. In this way, the SDSS data themselves can, in principle, be used to correct for the effects of the fixed aperture of the SDSS spectrograph.

In practice, because there is substantial scatter in the velocity dispersions at fixed luminosity and size (Paper II shows that this scatter is about $14 \%$ ), the trends in the present data set are relatively noisy. When the data set is larger, it will be worth returning to this issue. For now, because the corrections are small anyway, we have chosen to use equation (1) to correct the velocity dispersions. Nevertheless, curves like those presented above provide a novel way to study the velocity dispersion profiles of earlytype galaxies.

\section{APPENDIX D}

\section{ERROR ESTIMATES}

Let $\mathscr{E}$ denote the covariance matrix of the errors in our estimates of the absolute magnitude $M$, the half-light radius $R \equiv \log R_{o}$, and the velocity dispersion $V \equiv \log \sigma$ :

$$
\mathscr{E}=\left(\begin{array}{ccc}
\epsilon_{\mathrm{MM}}^{2} & \epsilon_{\mathrm{RM}}^{2} & \epsilon_{\mathrm{VM}}^{2} \\
\epsilon_{\mathrm{RM}}^{2} & \epsilon_{\mathrm{RR}}^{2} & \epsilon_{\mathrm{RV}}^{2} \\
\epsilon_{\mathrm{VM}}^{2} & \epsilon_{\mathrm{RV}}^{2} & \epsilon_{\mathrm{VV}}^{2}
\end{array}\right) .
$$

The elements of the error matrix $\mathscr{E}$ are obtained as follows.

The photometric pipeline estimates the size $r_{\mathrm{dev}}$ and the apparent magnitude $m_{\mathrm{dev}}$ from the same fitting procedure. As a result, errors in these two quantities are correlated. Let $e_{r}$ denote the error in $\log r_{\mathrm{dev}}$, and $e_{m}$ the error in $m_{\mathrm{dev}}$. The correlation means that we need three numbers to describe the errors associated with the fitting procedure, $\left\langle e_{r} e_{r}\right\rangle$, $\left\langle e_{m} e_{m}\right\rangle$, and $\left\langle e_{m} e_{r}\right\rangle$, but the pipeline only provides two. The error output by the pipeline in $r_{\text {dev }}$ is correctly marginalized over the uncertainty in $m_{\mathrm{dev}}$, so it is essentially $\left\langle e_{r} e_{r}\right\rangle$. On the other hand, the quoted error in $m_{\mathrm{dev}}$, say $\left\langle e_{\text {photo }}^{2}\right\rangle$ is really $\left\langle e_{m} e_{m}\right\rangle-\left\langle e_{m} e_{r}\right\rangle^{2} /\left\langle e_{r} e_{r}\right\rangle$. To estimate the values of $\left\langle e_{m} e_{r}\right\rangle$ and $\left\langle e_{m} e_{m}\right\rangle$ that we need, we must make an assumption about the correlation between the errors.

Fortunately, this can be derived from the fact that, for a wide variety of galaxy profile shapes, the quantity $\xi \equiv e_{r}-\alpha e_{\mu}$, with $\alpha \approx 0.3$, has a very small scatter (e.g., Saglia et al. 1997). Here $\mu \equiv m_{\mathrm{dev}}+5 \log r_{\mathrm{dev}}+2.5 \log 2 \pi$ is the surface brightness, and $e_{\mu}$ is the error in the surface brightness. As a result,

$$
\begin{aligned}
& \left\langle e_{\mu} e_{\mu}\right\rangle=\frac{\left\langle e_{r}^{2}\right\rangle}{\alpha^{2}}+\frac{\left\langle\xi^{2}\right\rangle}{\alpha^{2}} \frac{\left(\alpha^{2}-1\right)}{\left(1+\alpha^{2}\right)}, \\
& \left\langle e_{\mu} e_{r}\right\rangle=\frac{\left\langle e_{r}^{2}\right\rangle}{\alpha}-\frac{\left\langle\xi^{2}\right\rangle}{\alpha\left(1+\alpha^{2}\right)}
\end{aligned}
$$

(Saglia et al. 1997). This means that $\left\langle e_{r} \xi\right\rangle=\left\langle\xi^{2}\right\rangle /\left(1+\alpha^{2}\right)$, so that

$$
\begin{aligned}
& \left\langle e_{m} e_{r}\right\rangle=\left\langle e_{r} e_{r}\right\rangle \frac{1-5 \alpha}{\alpha}-\frac{\left\langle\xi^{2}\right\rangle}{\alpha\left(1+\alpha^{2}\right)}, \\
& \left\langle e_{m} e_{m}\right\rangle=\left\langle e_{r} e_{r}\right\rangle\left(\frac{1-5 \alpha}{\alpha}\right)^{2}+\frac{\left\langle\xi^{2}\right\rangle}{\alpha^{2}}\left[1-2\left(\frac{1-5 \alpha}{1+\alpha^{2}}\right)\right], \\
& \left\langle e_{m} e_{m}\right\rangle-\frac{\left\langle e_{m} e_{r}\right\rangle^{2}}{\left\langle e_{r} e_{r}\right\rangle}=\frac{\left\langle\xi^{2}\right\rangle}{\alpha^{2}}-\frac{\left\langle\xi^{2}\right\rangle}{\alpha^{2}} \frac{\left\langle\xi^{2}\right\rangle /\left\langle e_{r} e_{r}\right\rangle}{\left(1+\alpha^{2}\right)^{2}}=\left\langle e_{\text {photo }}^{2}\right\rangle .
\end{aligned}
$$

The final equality shows that the error output from the pipeline provides an estimate of $\left\langle\xi^{2}\right\rangle$ that we can insert into our expressions for $\left\langle e_{m} e_{m}\right\rangle,\left\langle e_{m} e_{r}\right\rangle$, and $\left\langle e_{\mu} e_{\mu}\right\rangle$. (Note that if $\left\langle\xi^{2}\right\rangle \ll\left\langle e_{r} e_{r}\right\rangle$, then it would be a good approximation to set $\left\langle e_{\text {photo }}^{2}\right\rangle \approx\left\langle\xi^{2}\right\rangle / \alpha^{2}$. Since this is not always the case for our data set, we must solve the quadratic.) Once this has been done, we set

$$
\begin{aligned}
\epsilon_{\mathrm{MM}}^{2} & =\left\langle e_{m} e_{m}\right\rangle, \\
\epsilon_{\mathrm{RR}}^{2} & =\left\langle e_{r} e_{r}\right\rangle+\left(\left\langle e_{\mathrm{ab}} e_{\mathrm{ab}}\right\rangle / 4\right), \\
\epsilon_{\mathrm{RM}}^{2} & =\left\langle e_{m} e_{r}\right\rangle, \\
\epsilon_{\mathrm{VM}}^{2} & =0, \\
\epsilon_{\mathrm{VV}}^{2} & =\left\langle e_{v} e_{v}\right\rangle+\left(0.04 \epsilon_{\mathrm{RR}}\right)^{2}, \\
\epsilon_{\mathrm{RV}}^{2} & =-0.04 \epsilon_{\mathrm{RR}}^{2} .
\end{aligned}
$$

That is, we compute the error in the absolute magnitude by assuming that there are no errors in the determination of the redshift (and $K$-correction!) that would otherwise propagate through.

Subsequent papers will focus almost exclusively on the circularly averaged radius $R_{o}$ defined in $\S 3.2 .1$. The errors on it are given by adding the errors in the size $r_{\mathrm{dev}}$ to those that come from the error on the shape $b / a$. We assume that the errors in $b / a$ are neither correlated with those in $\log r_{\mathrm{dev}}$ nor with those in the absolute magnitude. Finally, we assume that errors in magnitudes are not correlated with those in velocity dispersion, so $\left\langle\epsilon_{\mathrm{VM}}^{2}\right\rangle$ is set to zero, and that errors in size and velocity dispersion are only weakly correlated because of the aperture correction we apply. Here $\left\langle e_{v} e_{v}\right\rangle$ is the error in what was called $\log \sigma_{\text {est }}$ in the main text. These error estimates are presented in Tables 2 and 3, where we have set $\delta m_{\text {photo }}=\left\langle e_{m}^{2}\right\rangle^{1 / 2}, \delta_{r}=\left\langle e_{r}^{2}\right\rangle^{1 / 2}$, and $\delta M=\epsilon_{\mathrm{MM}}$. 
Baum, W. A. 1959, PASP, 71, 106

Bender, R. 1990, A\&A, 229, 441

Bender, R., Saglia, R. P., \& Gerhard, O. E. 1994, MNRAS, 269, 785

Benitez, N. 2000, ApJ, 536, 571

Bernardi, M., Alonso, M. V., da Costa, L. N., Willmer, C. N. A., Wegner, G., Pellegrini, P. S., Rité, C., \& Maia, M. A. G. 2002a, AJ, 123, 2159 2002b, AJ, 123, 2990

Bernardi, M., Renzini, A., da Costa, L. N., Wegner, G., Alonso, M. V.,

Pellegrini, P. S., Rité, C., \& Willmer, C. N. A. 1998, ApJ, 508, L143

Bernardi, M., et al. 2003a, AJ, (Paper II) 2003b, AJ, 125, 1866 (Paper III) 2003c, AJ, 125, 1882 (Paper IV)

Bingelli, B., Sandage, A., \& Tarenghi, M. 1984, AJ, 89, 64

Binney, J., \& Tremaine, S. 1987, Galactic Dynamics (Princeton: Princeton Univ. Press)

Blakeslee, J. P., Lucey, J. R., Barris, B. J., Hudson, M. J., \& Tonry, J. L. 2001, MNRAS, 327, 1004

Blanton, M. R., et al. 2001, AJ, 121, 2358

Bressan, A., Chiosi, C., \& Fagotto, F. 1994, ApJS, 94, 63

Brodie, J. P., \& Hanes, D. A. 1986, ApJ, 300, 258

Bruzual A., G., \& Charlot, S. 2003, in preparation

Burbidge, E. M., Burbidge, G. R., \& Fish, R. A. 1961, ApJ, 134, 251

Burstein, D., Bender, R., Faber, S. M., \& Nolthenius, R. 1997, AJ, 114 1365

Coleman, G. D., Wu, C.-C., \& Weedman, D. W. 1980, ApJS, 43, 393

Colless, M., Burstein, D., Davis, R. L., McMahan, R. K., Saglia, R. P., \& Wegner, G. 1999, MNRAS, 303, 813

Colless, M., Saglia, R. P., Burstein, D., Davies, R. L., McMahan, R. K., \& Wegner, G. 2001, MNRAS, 321, 277

Connolly, A. J., \& Szalay, A. S. 1999, AJ, 117, 2052

da Costa, L. N., Bernardi, M., Alonso, M. V., Wegner, G., Willmer,

C. N. A., Pellegrini, P. S., Rité, C., \& Maia, M. A. G. 2000, AJ, 120, 95

de Vaucouleurs, G. 1948, Ann. d'Astrophys., 11, 247

de Zeeuw, T., \& Franx, M. 1991, ARA\&A, 29, 239

Diaz, A. I., Terlevich E., \& Terlevich, R. 1989, MNRAS, 239, 325

Djorgovski, S. \& Davis, M. 1987, ApJ, 313, 59

Dressler, A. 1984, ApJ, 286, 97

Dressler, A., Lynden-Bell, D., Burstein, D., Davies, R. L., Faber, S. M., Terlevich, R. J., \& Wegner, G. 1987, ApJ, 313, 42

Eisenstein, D. J., et al. 2001, AJ, 122, 2267

Ellis, R. S., Smail, I., Dressler, A., Couch, W. J., Oemler, A., Butcher, H., \& Sharples, R. M. 1997, ApJ, 483, 582

Faber, S. M., Dressler, A., Davies, R. L., Burstein, D., Lynden-Bell, D., Terlevich, R. J., \& Wegner, G. 1987, in Nearly Normal Galaxies: From the Planck Time to the Present, ed. S. M. Faber (New York: Springer), 175

Faber, S. M., \& Jackson, R. 1976, ApJ, 204, 668

Faber, S. M., Wegner, G., Burstein, D., Davies, R. L., Dressler, A., Lynden-Bell, D., \& Terlevich, R. J. 1989, ApJS, 69, 763

Fish, R. A. 1964, ApJ, 139, 284

Franx, M., Illingworth, G. D., \& Heckman, T. 1989, ApJ, 344, 613

Fukugita, M., Ichikawa, T., Gunn, J. E., Doi, M., Shimasaku, K., \& Schneider, D. P. 1996, AJ, 111, 1748

Fukugita, M., Shimasaku, K., \& Ichikawa, T. 1995, PASP, 107, 945

Giovanelli, R., Haynes, M. P., Herter, T., Vogt, N. P., da Costa, L. N. Freudling, W., Salzer, J. J., \& Wegner, G. 1997, AJ, 113, 53

Gunn, J. E., et al. 1998, AJ, 116, 3040

Hernquist, L. 1990, ApJ, 356, 359

Hogg, D. 1999, preprint (astro-ph/9905116)

Hogg, D. W., Schlegel, D. J., Finkbeiner, D. P., \& Gunn, J. E. 2001, AJ 122,2129

Jørgensen, I., Franx, M., \& Kjærgaard, P. 1995, MNRAS, 276, 1341

. 1996, MNRAS, 280, 167

Kauffmann, G. 1996, MNRAS, 281, 487

Kauffmann, G., \& Charlot, S. 1998, MNRAS, 294, 705

Kelson, D. D., Illingworth, G. D., van Dokkum, P. G., \& Franx, M. 2000 ApJ, 531, 184

Kochanek, C. S., Pahre, M. A., \& Falco, E. E. 2000, ApJ, submitted (astro$\mathrm{ph} / 0011458)$
Kormendy, J. 1977, ApJ, 218, 333

. 1982, Morphology and Dynamics of Galaxies, ed L. Martinet \& M. Mayor (Sauverny: Geneva Obs.), 115

Kroupa, P. 2001, in ASP Conf. Ser. 228, Dynamics of Star Clusters and the Milky Way, ed. S. Deiters, B. Fuchs, R. Spurzem, A. Just, \& R. Wielen (San Francisco: ASP), 187

Kuntschner, H., Lucey, J. R., Smith, R. J., Hudson, M. J., \& Davies, R. L. 2001, MNRAS, 323, 615

Larson, R. B. 1975, MNRAS, 173, 67

Lin, H., Yee, H. K. C., Carlberg, R. G., Morris, S. L., Sawicki, M., Patton, D. R., Wirth, G., \& Shepherd, C. W. 1999, ApJ, 518, 533

Lupton, R., Gunn, J. E., Ivezić, Z., Knapp, G. R., \& Kent, S. 2001, in ASP Conf. Ser. 238, Astronomical Data Analysis Software and Systems X, ed. F. R. Harnden, Jr., F. A. Primini, \& H. E. Payne (San Francisco: ASP), 269

Madgwick, D. S., et al. 2002, MNRAS, 333, 133

Pahre, M. A. 1998, PASP, 110, 1249

Pahre, M. A., de Carvalho, R. R., \& Djorgovski, S. G. 1998a, AJ, 116, 1606

Pahre, M. A., Djorgovski, S. G., \& de Carvalho, R. R. 1998b, AJ, 116, 1591

Petrosian, V. 1976, ApJ, 209, L1

Rix, H.-W., \& White, S. D. M. 1992, MNRAS, 254, 389

Roberts, M. S., \& Haynes, M. P. 1994, ARA\&A, 32, 115

Saglia, R. P., Bertschinger, E., Baggley, G., Burstein, D., Colless, M., Davies, R. L., McMahan, Jr., R. K., \& Wegner, G. 1993, MNRAS, 264 961

1997, ApJS, 109, 79

Saglia, R. P., Colless, M., Burstein, D., Davies, R. L., McMahan, R. K., \& Wegner, G. 2001, MNRAS, 324, 389

Sargent, W. L. W., Schechter, P. L., Boksenberg, A., \& Shortridge, K. 1977, ApJ, 212, 326

Schade, D., et al. 1999, ApJ, 525, 31

Schlegel, D. J., Finkbeiner, D. P., \& Davis, M. 1998, ApJ, 500, 525

Scodeggio, M., Gavazzi, G., Belsole, E., Pierini, D., \& Boselli, A. 1998, MNRAS, 301, 1001

Smith, J. A., et al. 2002, AJ, 123, 2121

Stoughton, C., et al. 2002, AJ, 123, 485

Strauss, M. A., et al. 2002, AJ, 124, 1810

Tantalo, R., Chiosi, C., \& Bressan, A. 1998, A\&A, 333, 419

Tinsley, B. M., \& Gunn, J. E. 1976, ApJ, 206, 525

Tonry, J., \& Davis, M. 1979, AJ, 84, 1511

Trager, S. C., Faber, S. M., Worthey, G., \& González, J. J. 2000a, AJ, 119 1645

2000b, AJ, 120, 165

Trager, S. C., Worthey, G., Faber, S. M., Burstein, D., \& González, J. J. 1998, ApJS, 116, 1

Treu, T., Stiavelli, M., Bertin, G., Casertano, S., \& Møller, P. 2001a, MNRAS, 326, 237

Treu, T., Stiavelli, M., Casertano, S., Møller, P., \& Bertin, G. 1999, MNRAS, 308, 1037

Treu, T., Stiavelli, M., Møller, P., Casertano, S., \& Bertin, G. 2001b, MNRAS, 326, 221

Tully, R. B., \& Fisher, J. R. 1977, A\&A, 54, 66

van Albada, T. S. 1982, MNRAS, 201, 939

van der Marel, R. P., \& Franx, M. 1993, ApJ, 407, 525

van Dokkum, P. G., \& Franx, M. 1996, MNRAS, 281, 985

van Dokkum, P. G., Franx, M., Kelson, D. D., \& Illingworth, G. D. 1998 ApJ, 504, L17

2001, ApJ, 553, L39

Vazdekis, A., Casuso, E., Peletier, R. F., \& Beckman, J. E. 1996, ApJS, 106, 307

Wegner, G., Colless, M., Saglia, R. P., McMahan, R. K., Davies, R. L., Burstein, D., \& Baggley, G. 1999, MNRAS, 305, 259

Weinberg, S. 1972, Gravitation and Cosmology: Principles and Applications of the General Theory of Relativity (New York: Wiley)

White, S. D. M., \& Rees, M. J. 1978, MNRAS, 183, 341

Worthey, G. 1994, ApJS, 95, 107

York, D. G., et al. 2000, AJ, 120, 1579

Ziegler, B. L., Bower, R. G., Smail, I., Davies, R. L., \& Lee, D. 2001, MNRAS, 325, 1571 